\title{
EXPLORING DATA DRIVEN MODELS OF TRANSIT TRAVEL TIME AND DELAY
}

\author{
A Thesis \\ presented to \\ the Faculty of California Polytechnic State University, \\ San Luis Obispo \\ In Partial Fulfillment \\ Of the Requirements for the Degree \\ Master of Science in Civil \& Environmental Engineering \\ by
}

Bobjot Singh Sidhu

June 2016 
(C) 2016

Bobjot Singh Sidhu

ALL RIGHTS RESERVED 
TITLE: Exploring Data Driven

Models of Transit Travel Time and Delay

\author{
AUTHOR: $\quad$ Bobjot Singh Sidhu
}

DATE SUBMITTED: June 2016

COMMITTEE CHAIR: Robert L. Bertini, Ph.D.

Associate Professor of Civil and Environmental Engineering

COMMITTEE CHAIR: $\quad$ Anurag Pande, Ph.D.

Associate Professor of Civil and Environmental Engineering

COMMITTEE MEMBER: Kimberley Mastako, Ph.D.

Lecturer of Civil and Environmental Engineering 


\section{ABSTRACT \\ Exploring Data Driven Models of Transit Travel Time and Delay \\ Bobjot Singh Sidhu}

Transit travel time and operating speed influence service attractiveness, operating cost, system efficiency and sustainability. The Tri-County Metropolitan Transportation District of Oregon (TriMet) provides public transportation service in the tri-county Portland metropolitan area. TriMet was one of the first transit agencies to implement a Bus Dispatch System (BDS) as a part of its overall service control and management system. TriMet has had the foresight to fully archive the BDS automatic vehicle location and automatic passenger count data for all bus trips at the stop level since 1997. More recently, the BDS system was upgraded to provide stop-level data plus 5-second resolution bus positions between stops. Rather than relying on prediction tools to determine bus trajectories (including stops and delays) between stops, the higher resolution data presents actual bus positions along each trip. Bus travel speeds and intersection signal/queuing delays may be determined using this newer information.

This thesis examines the potential applications of higher resolution transit operations data for a bus route in Portland, Oregon, TriMet Route 14. BDS and 5-second resolution data from all trips during the month of October 2014 are used to determine the impacts and evaluate candidate trip time models. Comparisons are drawn between models and some conclusions are drawn regarding the utility of the higher resolution transit data.

In previous research inter-stop models were developed based on the use of average or maximum speed between stops. We know that this does not represent realistic conditions of stopping at a signal/crosswalk or traffic congestion along the link. A new inter-stop trip time model is developed using the 5-second resolution data to determine 
the number of signals encountered by the bus along the route. The variability in inter-stop time is likely due to the effect of the delay superimposed by signals encountered. This newly developed model resulted in statistically significant results. This type of information is important to transit agencies looking to improve bus running times and reliability. These results, the benefits of archiving higher resolution data to understand bus movement between stops, and future research opportunities are also discussed. 


\section{ACKNOWLEDGMENTS}

No great achievement is ever completed alone. I owe thanks to numerous people for their help and support in the development of this thesis. First and foremost, I would like to thank my advisors, Dr. Robert Bertini and Dr. Anurag Pande, for their dedication to the success of this research. Dr. Bertini's expertise in transit trip time and dwell time models provided great insight and allowed me to build on the previous methods with the higher resolution data. Also, his connections allowed me to work with real archived data and discuss various methodologies with his expert contacts. Special thanks to Steve Callas for providing a month of archived transit data from TriMet. I thankfully acknowledge Dr. Pande for being my co-advisor and helping build my thesis. Many thanks also go to Dr. Mastako for her thoughtful guidance and for enhancing my interest in transportation. Her passion and knowledge in my traffic engineering class encouraged me to concentrate in transportation. I want to thank Drs. Bertini, Pande and Mastako for volunteering to serve on my thesis committee.

I am appreciative of all the friends that I have made over the last 6 years. The people I've met and the perspective I've gained has provided me with relationships that will extend far beyond our time in undergraduate/graduate school. This journey may have taken longer than I anticipated, but I am grateful for all of the hard work and memories!

On a personal note, I would like to thank my extraordinary parents, Inderjit and Pardeep Sidhu, my brother Manjot Sidhu, and my grandmother, Surjeet Kaur, for their love, support and financial investments in my education. I am extremely grateful for the opportunity to pursue my dreams and it would not be possible without my family. 


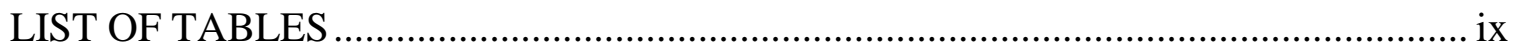

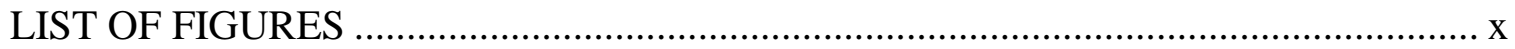

\section{CHAPTER}

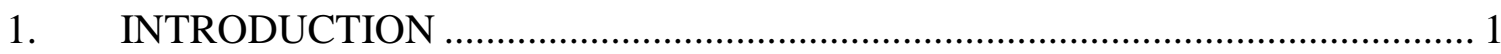

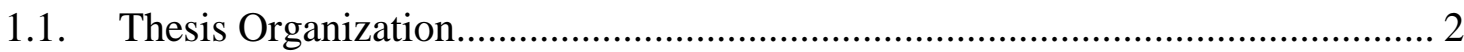

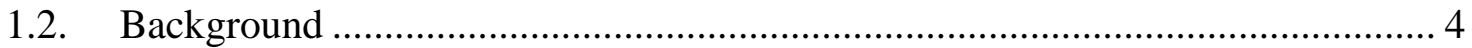

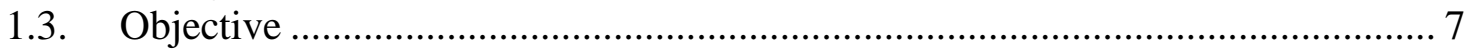

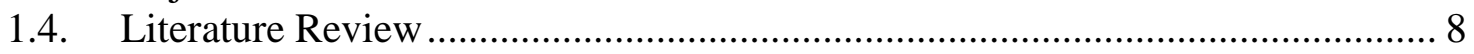

1.4.1. Overview of AVL Systems ................................................................ 8

1.4.2. Decision Tree Models ......................................................................... 9

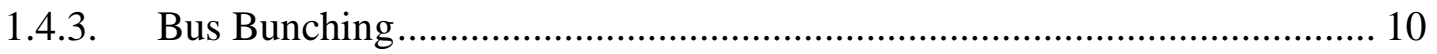

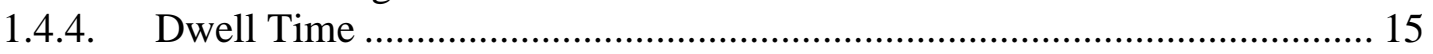

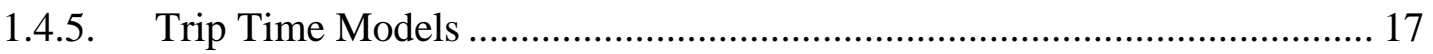

1.4.6. 5-Second Resolution Data....................................................................... 19

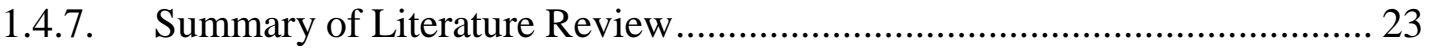

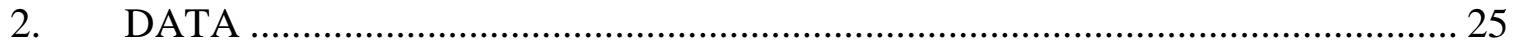

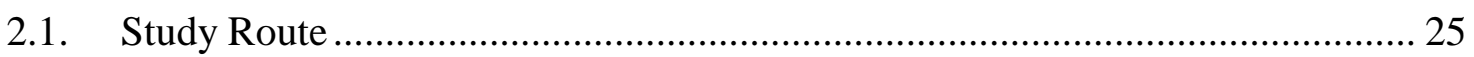

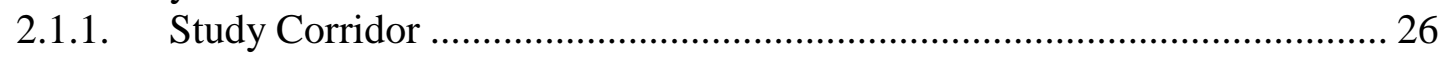

2.2. Bus Dispatch System (BDS) Data.............................................................. 28

2.2.1. Data Preparation............................................................................... 33

2.2.2. Passenger Movement Data....................................................................... 35

2.2.3. Headway Data Preparation ……………………................................... 38

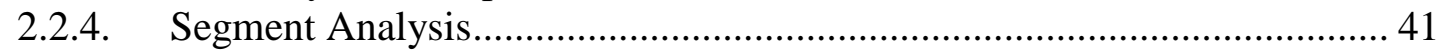

2.2.5. Travel Time Determinants Preparation...................................................... 42

2.2.6. Newell Trip Time Model Data Preparation ................................................. 44

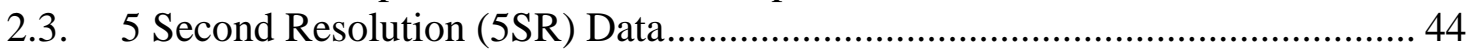

2.3.1. 5 Second Data Preparation ....................................................................... 46

2.3.2. Travel Speed Analysis ......................................................................... 49

2.3.3. Acceleration and Deceleration ............................................................... 54

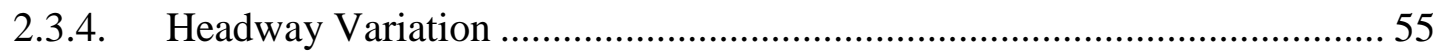

2.3.5. Average vs Actual Trajectory ................................................................. 58

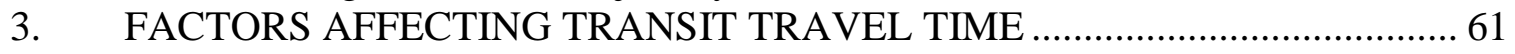

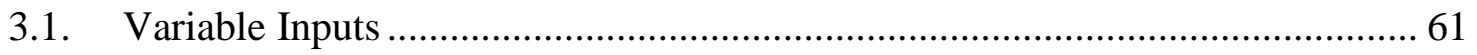

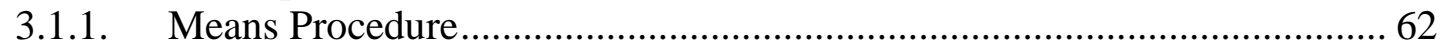

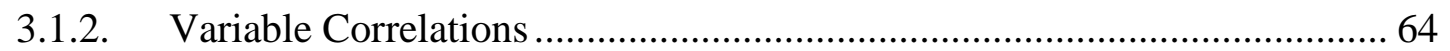

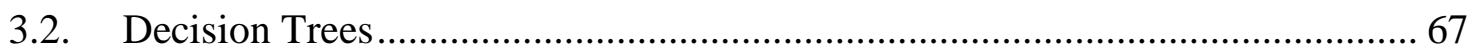

4. TRANSIT TRIP TIME MODEL ................................................................ 72

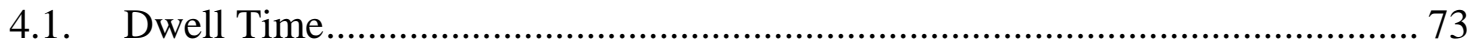

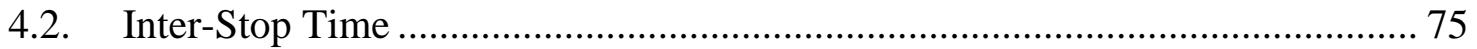

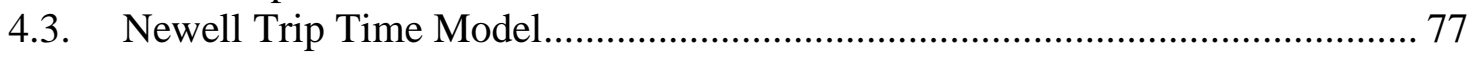

4.4. New Inter-Stop Model................................................................................. 78 
4.4.1. Trip Time Model 1: Random Events ................................................. 79

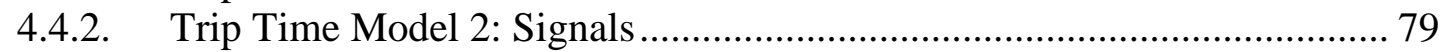

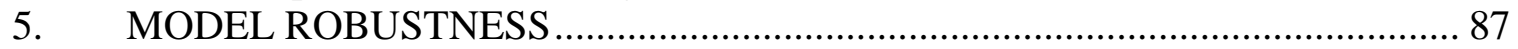

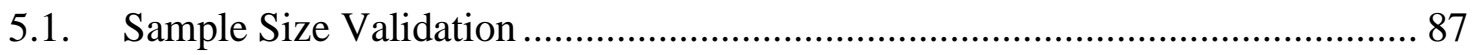

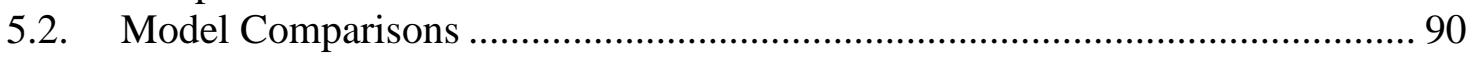

5.2.1. Paired t-Test: Difference of Means ................................................. 91

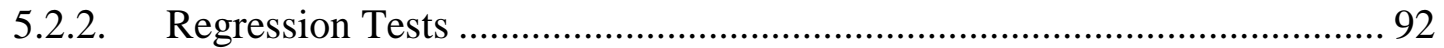

5.2.3. Root Mean Squared Error ............................................................... 93

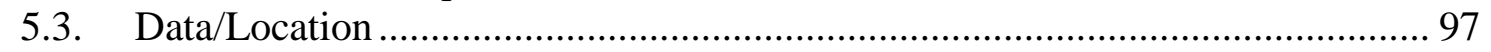

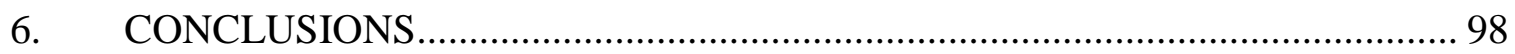

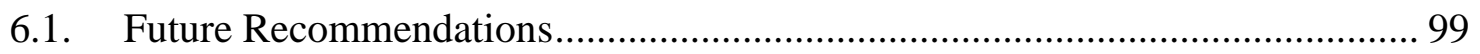

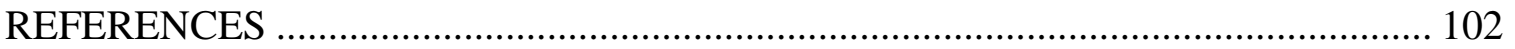

\section{APPENDICES}

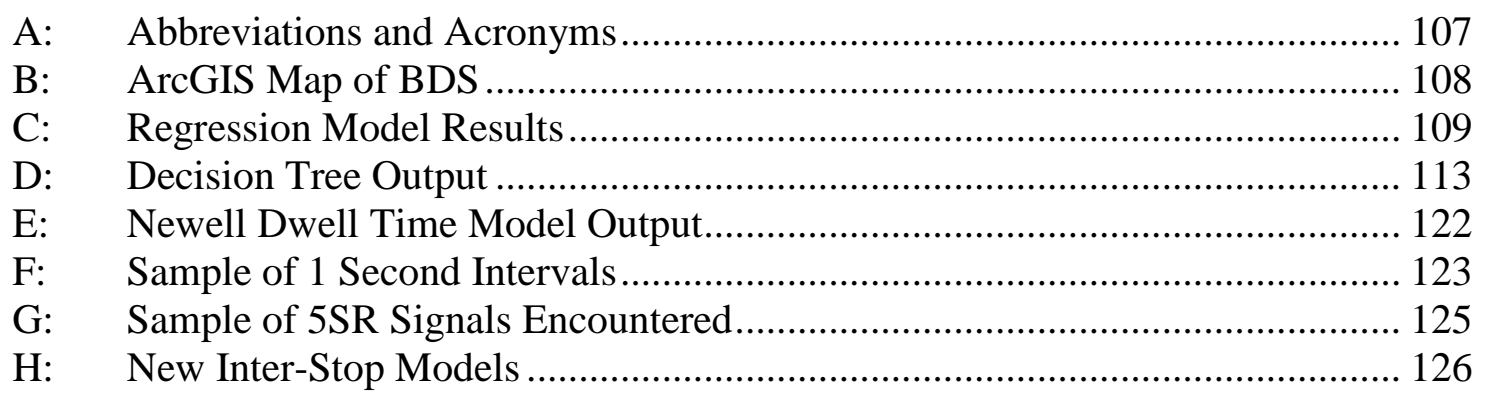




\section{LIST OF TABLES}

Table

Table 1: Number of Signalized/Unsignalized Segments along Route 14 ..................... 28

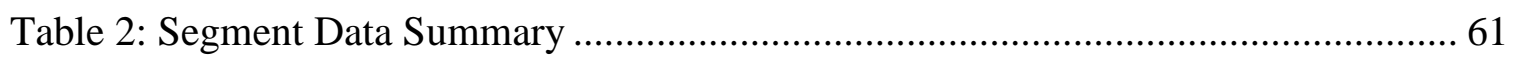

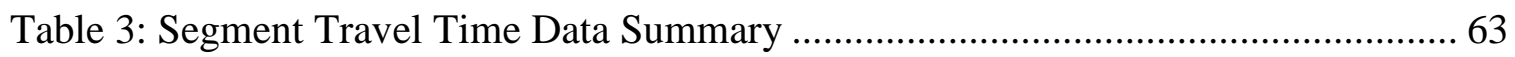

Table 4: Newell Trip Time Model Summary for 2003 and 2014 _.............................. 78

Table 5: Individual Inter-Stop Data Summary for 1 Week......................................... 80

Table 6: Total Inter-Stop Trip Time Characteristics for 1 Week................................. 80

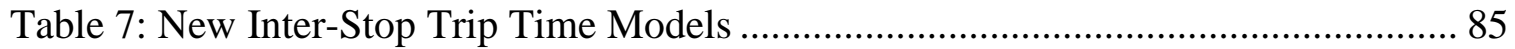

Table 8: Maximum Error Estimation at 95\% Confidence Intervals ............................. 88

Table 9: Minimum Sample Size Requirement for 95\% Confidence Intervals ................ 89

Table 10: Paired T-Test for Newell and New Inter-Stop Model ................................. 91

Table 11: New Inter-Stop Model Summary Output .............................................. 92

Table 12: New Inter-Stop Model Regression Statistics ............................................. 93 


\section{LIST OF FIGURES}

Figure

Page

Figure 1: TriMet's Bus Ridership 2008-2013 (TriMet, 2016)..................................... 5

Figure 2: On-Time Performance Chart from TriMet, (TriMet, 2016) ............................ 6

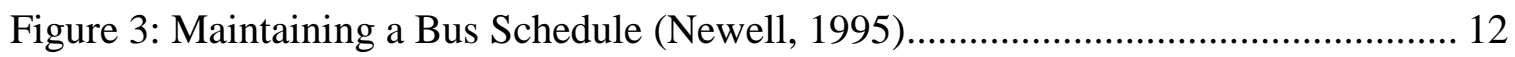

Figure 4: Forecasted Trajectories using Particle Filter (Hans et al., 2015) ..................... 13

Figure 5: Diagram of Non Dwell Time and Dwell Time Split ................................... 16

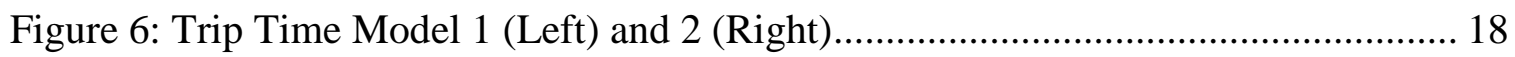

Figure 7: Speed Comparison of 5SR vs BDS (Glick et al., 2015) ................................. 20

Figure 8: Heat Map of Speeds on Powell Blvd using 5SR (Stoll et al., 2016) ................ 21

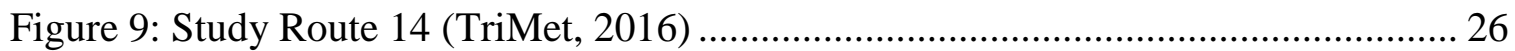

Figure 10: Google Maps Photo of Route 14 with Signalized Intersections..................... 27

Figure 11: Components of BDS Data (Tantiyanugulchai, 2004) ................................. 29

Figure 12: Stop Circle where the BDS Records Times and Locations ........................... 31

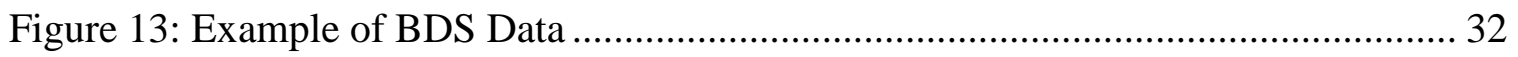

Figure 14: Trip Distance Comparison of BDS and Google Distance ............................ 34

Figure 15: Example of Bus Trajectories on a Time-Space Diagram ............................ 35

Figure 16: Passenger Load for AM Peak Period using BDS Data ................................ 36

Figure 17: Passenger Movement and Time Space Diagram of 2 Trips ......................... 37

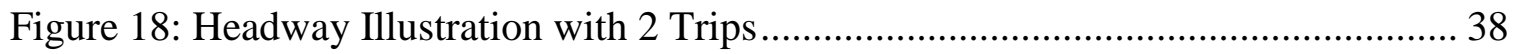

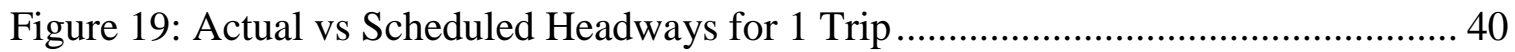

Figure 20: Inter-Stop Segment on Hawthorne Blvd .............................................. 41

Figure 21: Segment between Critical Time Points on Hawthorne Blvd......................... 42 
Figure 22: Methodology for Decision Tree Models 43

Figure 23: Example of 5SR Data 45

Figure 24: Verification of 5SR Data with Stop Level Data......................................... 47

Figure 25: Raw Stop Level Data vs 5SR Data (Top) Modified 5SR Data (Bottom)....... 48

Figure 26: Average Speed Plot for AM Peak Period ................................................. 50

Figure 27: Average Speed Histogram using BDS Data........................................... 51

Figure 28: Average Speed Histogram using 5SR Data............................................ 52

Figure 29: Inter-Stop Time vs Dwell Time for Segment ........................................... 54

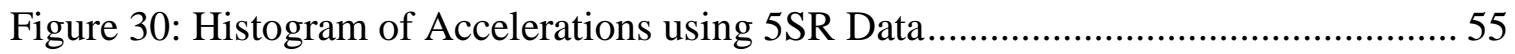

Figure 31: Headway Comparison of Stop Level Data vs Raw 5SR Data....................... 56

Figure 32: Headway for 2 Trips using Stop Level Data (Top) 5SR Data (Bottom) ........ 57

Figure 33: Stop Level Data vs Modified 5SR Data for 1 Trip..................................... 59

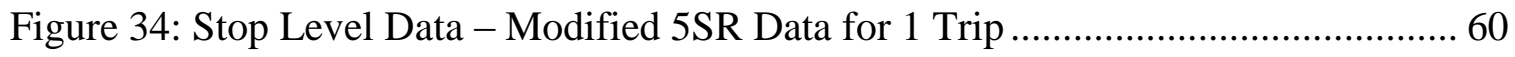

Figure 35: Segment Travel Time for each Time Period ......................................... 63

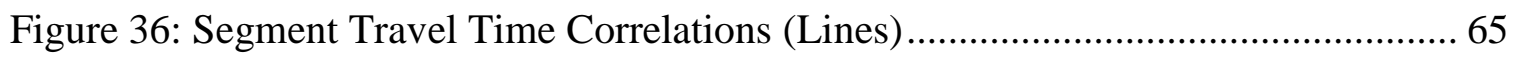

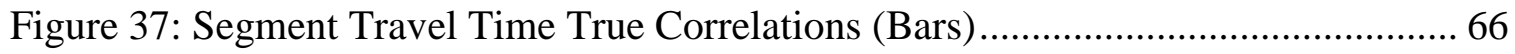

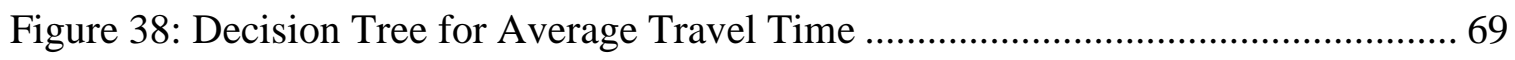

Figure 39: Decision Tree for Travel Time Excluding Dwell Time ............................... 70

Figure 40: Inter-Stop Time vs Dwell Time for First Mile of Trip .............................. 73

Figure 41: Dwell Time vs Passenger Movement for 1 Week ...................................... 74

Figure 42: Boxplot of Trip Time vs Distance for First Mile of Trip ............................ 75

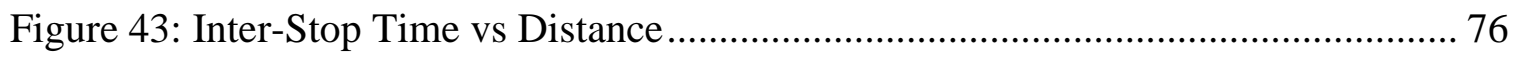

Figure 44: Signals Encountered for 1 Trip with BDS and 5SR ............................... 81 
Figure 45: Signals Encountered for 1 Trip at SE Powell Blvd ................................... 82

Figure 46: Google Maps Photo of Far-Side Bus Stop at SE Powell Blvd ....................... 83

Figure 47: Signals Encountered vs Total Inter-Stop Time for All Day .......................... 84

Figure 48: Signals Encountered vs Inter-Stop Time AM Peak Period .......................... 85

Figure 49: Minimum Number of Observations Based on Maximum Error.................... 90

Figure 50: Predicted vs Actual Plot for Newell (Top) New Inter-Stop Model (Bottom). 95

Figure 51: Residual Plot for Newell and New Inter-Stop Models............................... 96 


\section{INTRODUCTION}

Traffic congestion has become a serious problem, especially in urban areas around the world. Not only does congestion reduce the efficiency of transportation infrastructure, it also increases travel time, air pollution and fuel consumption. As roads become more congested with private automobiles, the shift towards sustainable mobility becomes more important. A desirable strategy to alleviate congestion is to shift more people from private automobiles to public transit by providing better transit service. Public transportation is an integral part of the solution to improving traffic conditions and reducing emissions. However, transit reliability needs to be improved to attract more ridership. Transit travel time and schedule adherence are important to transit agencies and to passengers (NCTR, 2005) and can have a cyclical effect-as performance improves, service is more attractive and competitive.

Transit agencies are increasingly basing performance measurement, traveler information and proactive management strategies on systems that include automatic vehicle location (AVL) and automatic passenger counters (APC). If archived, these data can provide a valuable historical perspective that enhances planning and operational improvement tracking. Past AVL/APC systems primarily emphasized collecting (and archiving) data at the stop level. Now, however, even higher resolution data that can track bus movements between stops are now available and, if archived, may help improve planning and operational analysis. In particular, a recent Transit Cooperative Research Program (TCRP) project recommends the collection of data every 2 seconds in order to study a transit vehicle's path in greater detail. Additionally, TCRP states that frequent inter-stop records provide information about speed and acceleration (TCRP 113, 2006). 
Toward understanding the value of higher resolution transit data, this thesis utilized archived transit data from the Tri-County Metropolitan Transportation District of Oregon (Tri-Met) in the form of Bus Dispatch System (BDS) data and 5-second resolution (5SR) data for the month of October, 2014. Both of these datasets will be compared through various analyses, such as headway, speed, acceleration and trajectory variation. The higher resolution data presents valuable information that describes what occurs between stops. This newer and more detailed data may help transit agencies improve inter-stop links with transit signal priority, designated bus lanes or improved bus turnouts.

Among other real time operational benefits, the BDS provides valuable information about passenger movement and dwell times at the stops. Transit travel time variables which significantly impact travel time will be assessed using this data. Next, based on prior research, a classical transit trip time model will be recreated and evaluated using 2014 data. Potential applications of the higher resolution 5SR data will be explored. The 5SR data will be incorporated to develop an improved trip time model. Statistical analyses will be used to compare the trip time models. This thesis provides route-level performance measures based on travel time and scheduled headway to that can help transit agencies and operators predict trip times and identify locations that adversely affect transit reliability.

\subsection{Thesis Organization}

This thesis is divided into the following six chapters:

- Chapter 1 - Introduction: This chapter provides the background including: an overview of the objective and a detailed literature review. 
- Chapter 2 - Data: This chapter explains the study route, corridor and two data sets used for this study. An overview of BDS and 5SR data is included in this chapter. This chapter describes the preliminary data preparation for the analyses. The speeds and headways are analyzed along the route using both datasets. Graphical representations are created, including speed contour plots. Also, comparisons are made using both datasets, broken down by time of day.

- Chapter 3 - Factors Affecting Transit Travel Time: This chapter filters the stop level data to determine the various factors affecting bus travel time. A statistical software is used to test two weeks of data and graphical representations include correlation bar charts and decision trees.

- Chapter 4 - Transit Trip Time Model: This chapter redevelops previous trip time models using current data and the new, higher resolution data. This chapter includes graphical representations of each model.

- Chapter 5 - Model Robustness: This chapter describes some limitations of the data and validates the minimum sample size requirements. Also, the trip time models developed in the previous chapters are compared using statistical techniques for two sample means.

- Chapter 6 -Conclusions: Finally, the thesis concludes with a summary of findings, evaluation of the new, higher resolution data and an overview of the need for further research and limitations of the data. This chapter notes additional uses for archived transit data. 


\subsection{Background}

As congestion continues to increase, it becomes more imperative to reduce the number of vehicles on the road. The most efficient means is to shift users from private autos to public transit. Archived transit data can support data driven decisions that benefit transit users and transit agencies to be able to improve on-time performance and other performance goals. Most existing transit AVL systems are used primarily for managing transit operations in real-time. In Portland, Oregon, the Tri-County Metropolitan Transportation District of Oregon (TriMet) provides transit service in the metropolitan area. With almost 80 lines, buses serve much of the Portland metro area. Each bus is equipped with a bus dispatch system (BDS) which includes several stop-level data archiving capabilities (Strathman et al., 2003). There were over 1 million weekly boardings throughout 2014, and over 60 million trips by the transit buses in 2014. As shown in Figure 1 below, ridership has declined somewhat in recent years, likely due to service cuts during 2009-10, but needs to shift back up. 


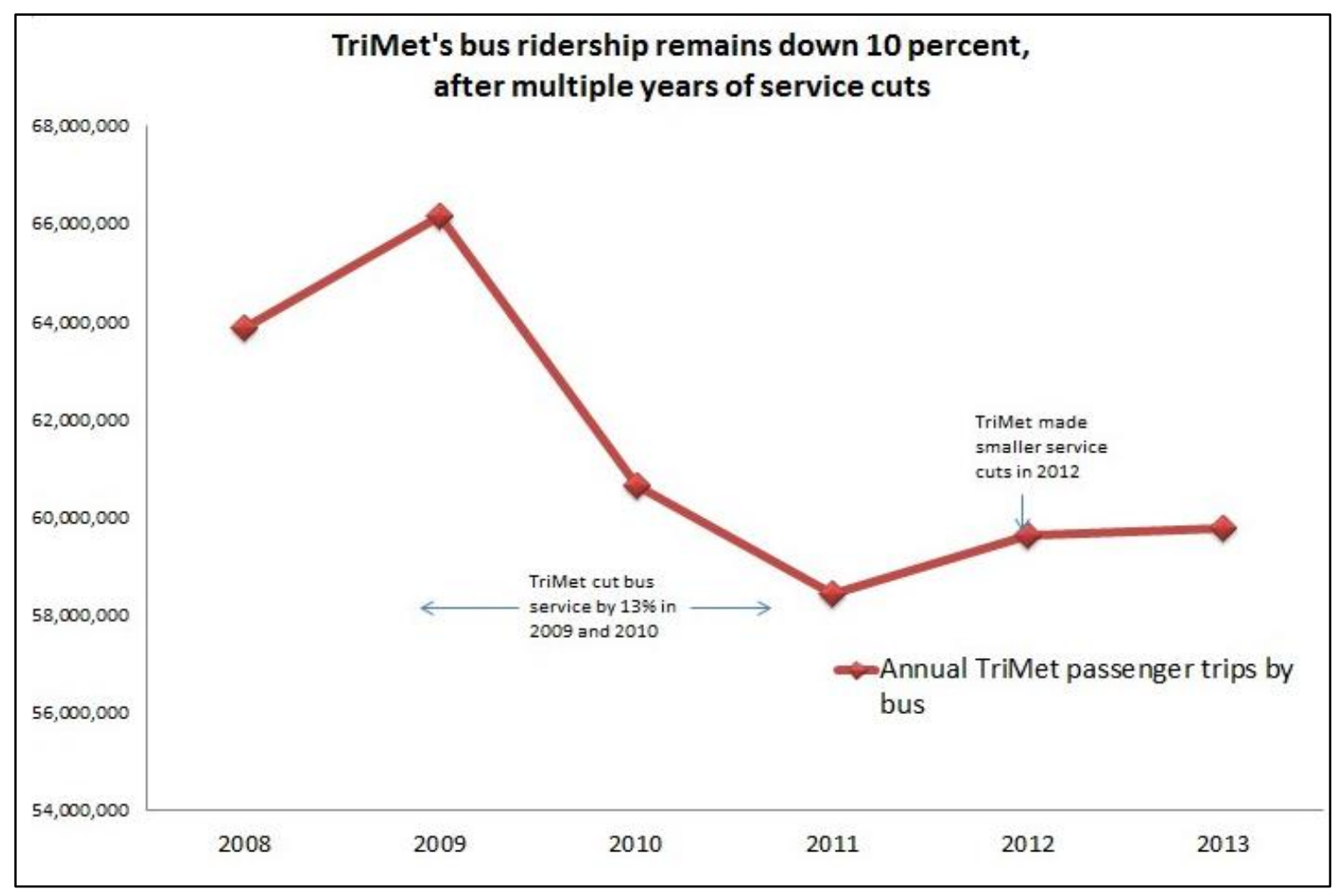

Figure 1: TriMet's Bus Ridership 2008-2013 (TriMet, 2016)

In order to curb congestion and continue to move forward, TriMet wants to invest in more and better transit service. The entire community in Portland benefits from good transit: less congestion on roadways, less pollution, less reliance on automobiles, and more accessibility/mobility downtown. "The history of TriMet is steeped in Oregon's fabled pioneering spirit. Founded 45 years ago from the ashes of the bankrupt Rose City Transit, TriMet has been profoundly influential in shaping the growth and character of the Portland region. Through innovations in policy development, system design and technological advancement, the agency continues to set benchmarks for the transit industry at home and abroad" (TriMet, 2016). 


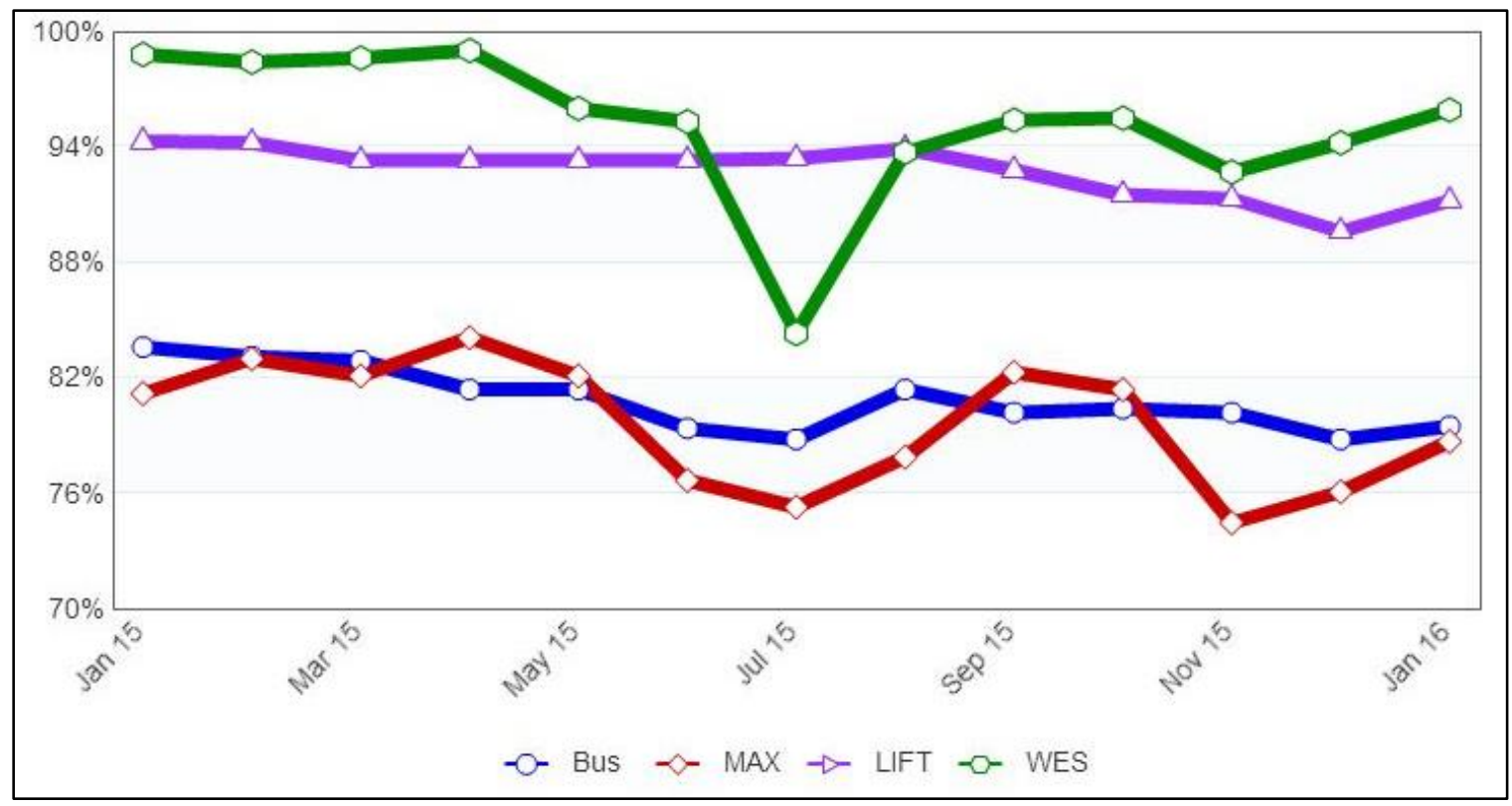

Figure 2: On-Time Performance Chart from TriMet, (TriMet, 2016)

As shown in Figure 2 the on-time performance is exceptional for TriMet buses. This figure includes a full years' worth of data and illustrates the on-time performances of TriMet's various public transportation services: buses, light rail (MAX), paratransit (LIFT) and commuter rail (WES). While $80 \%$ is an acceptable level for a transit service, this includes slack time. For buses and MAX lines, a vehicle is considered "on time" if it departs a scheduled time point no more than 1 minutes early and no more than 5 minutes late. Therefore, if this window becomes narrower the performance would decrease. However, during peak periods there is a higher frequency of buses which reduces the headway between buses and wait times for users. The higher frequency of buses would alleviate late buses.

In order to improve on-time performance, archived transit data is required for analysis. Initially, transit data had been collected using BDS at the stop-level. The 5 second resolution data system is global positioning satellite (GPS) based, transmitting data much more frequently than the previous fixed-route data system. This data and the 
BDS data are archived, providing rich sources of accurate time and location information supplemented by passenger information.

In addition to archived data, real-time information is imperative for transit agencies and passengers. Real-time information — whether provided through a display at a transit stop, by calling or texting an information service, or via a smartphone apphelps reassure passengers that their transit vehicle is on the way and can help them use their waiting time more efficiently. Studies in Seattle, the Netherlands and London found that on average, users with real-time information reported wait times that were $30 \%$, $20 \%$, and $65 \%$ lower, respectively. The majority of users also agreed that waiting time was more acceptable with the information (TCRP 165, 2013).

\subsection{Objective}

While many transit performance measures are only reported on an annual basis, performance measures can be analyzed for any timeframe and scale of transit operation. Motivated by the ongoing project, aimed at implementing the new higher resolution BDS in Portland, Oregon, the objective of this study is to improve transit travel time models and explore potential applications of higher resolution transit operations data. The Newell trip time model (Bertini \& El-Geneidy, 2004) is recreated and compared to a new interstop model, which incorporates the use of 5-second resolution data. This new, higher resolution data will also be used to identify bus bunching by observing the scheduled headways.

The study uses two key performance measures - travel time and speed-to compare the information available through the datasets. Chapter 2 contains a detailed description of the route and the two sources of transit data used in this study. 
Additionally, the route will be analyzed throughout the day to compare performance during different time periods. A decision tree analysis procedure will be used to explore the interaction between travel time and different variables. This process will provide a guided understanding of which variables affect travel time the most in order to construct and test statistical models based on those variables. All of the variables will be tested for statistical significance along with the trip time models.

\subsection{Literature Review}

To properly assess the potential of higher resolution transit data, a thorough understanding of the role of public transit within the realm of transportation is appropriate, along with assessing the functionality of automatic vehicle location systems in the context of public transit system operations. There has been a large amount of research on bus transit performance measures and improvements. Additionally, there are several studies that respond or build on the major works. This study focuses on the major work under AVL for transit services.

\subsubsection{Overview of AVL Systems}

The first widespread implementation of an automated passenger counter (APC) system in the United States began in 1982 at King County Metro. Ten years later in 1992, Metro acquired its first Automated Vehicle Location (AVL) and computer aided dispatch (CAD) system (Furth et al., 2006). TriMet has been archiving AVL data for all bus trips at the stop level since 1997. AVL describes the use of computers and global positioning systems (GPS) in dispatching and tracking transit vehicles. AVL systems have been increasingly utilized in the transit agencies as a means of tracking the locations of transit vehicles in real time (Casey et al., 1996). The potential use of AVL data can improve bus 
service planning and operations management (Furth et al., 2006). Although implementation of AVL slightly increases costs of operating and maintaining additional computer equipment, transit agencies benefit from improvements to customer service and real-time information (TCRP 73, 2008). Not only is the transit data available in real-time, but it is also archived for future research. Many operators have found that AVL has helped to improve service by increasing schedule adherence and enabling agencies to easily monitor bus driver performance. AVL also helps to reduce the response time to operational problems by improving communication between bus drivers and dispatchers. Passengers benefit from AVL systems with access to real-time bus arrival information. This real-time information means any information available to transit providers or users about the current status of vehicles, including approximate locations and predictive arrival times (NCTR, 2005).

AVL data helps researchers study the factors affecting bus travel time and service reliability at the route level, stop-to-stop segment level, and the time-point segment level (Strathman et al., 2001). Researchers have analyzed archived bus data statistically for travel-time delay, deviation and coefficient of variation (Bertini \& El-Geneidy, 2003). The transit industry is no exception from the trend toward increased use of technology, including wireless communication, automated vehicle location and sensors for counting passengers. As the trend continues, this paper will explore the potential of higher resolution data.

\subsubsection{Decision Tree Models}

Researchers examining public transportation systems generally agree on the key factors affecting travel time: trip distance, number of bus stops, road geometry, signalized 
intersections, time of day, passenger movements, fare payment method, vehicle characteristics, stop attributes and weather. More broadly, for multimodal transportation system performance measurement, important measures like average speed, travel time, and intersection delay can be used for performance monitoring of the transportation system (Bertini \& El-Geneidy, 2003). These measures are useful for system management, planning and for users. On freeways, these typical performance measures are often estimated directly using data from inductive loop detectors or other sensors (e.g., time mean speed, occupancy and vehicle counts). For arterials with numerous signalized intersections and access points, performance measures are more challenging due to more complicated traffic control and many origins and destinations. However, within signalized networks, travel time, speed, and other key performance measures can be obtained both directly and indirectly from sources such as AVL data (Bertini \& Tantiyanugulchai, 2004).

In order to distinguish which variables affect the travel time the most, archived transit data will be filtered and decision trees will be developed. Decision trees output the independent variables which influence the dependent variable. Classification and decision trees have been utilized in variety of transportation planning and engineering applications such as traffic safety, travel behavior, and red light violations (Pande \& Shaaban, 2015). Buses can become off-schedule due to surges in passenger arrivals leading to excessive dwell time, traffic conditions, and other random causes.

\subsubsection{Bus Bunching}

The stochastic nature of the transportation system environment exposes transit vehicles to incidents such as congestion, and varying passenger demand, which can have 
significant effects on headway regularity (Feng \& Figliozzi 2011). In the absence of system control strategies, it is common to observe bus bunching in transit operations. A general bus prediction framework was created to provide an accurate forecast of bus operations before the system becomes too disrupted to be restored to a stable condition (Hans et al., 2015). Reliability encompasses both on-time performance and the regularity of headways between successive transit vehicles. Uneven headways result in uneven passenger loadings. In the case of signaled rail operations, bunched trains often have to wait at track signals until the train ahead of them moves a safe distance forward. This is similar to bus bunching, when the preceding bus must wait for the leading bus to move ahead. The resulting unscheduled waits are not popular with passengers, particularly when no on board announcements are given explaining the delay (TCRP 165, 2013).

Models and concepts for transit operations by Newell and Potts (1964) are still relevant and applicable. Maintaining a regular schedule is important for satisfactory and efficient operation of a bus service. Frequency is a top factor in influencing overall trip satisfaction. For routes providing frequent service (headways of 10 minutes or less), the objective in schedule control is largely to ensure consistency in headways (time separation between vehicle arrivals or departures). Headways become shorter with more frequent service, consequently the arrivals become random. The most commonly used metric is the average passenger waiting time proposed by Newell and Potts (1964). Assuming uniform passenger arrival, average passenger waiting time is further derived as the sum of one half of the average headway and the ratio of headway variance to twice the average headway. Waiting times can be doubled as vehicles on frequent lines have a tendency to bunch. Headways on very frequent lines are inherently unstable: when a bus 
falls slightly behind schedule, it tends to pick up more passengers, causing it to slow further, until it eventually bunches with the trailing bus. The first effect is a slight delay at one stop, and then that offset amplifies. Alternate buses will continue to get delayed or ahead which creates off-scheduling and bus pairing as shown in Figure 3. This concept is illustrated using real BDS data in Chapter 2.2.1.

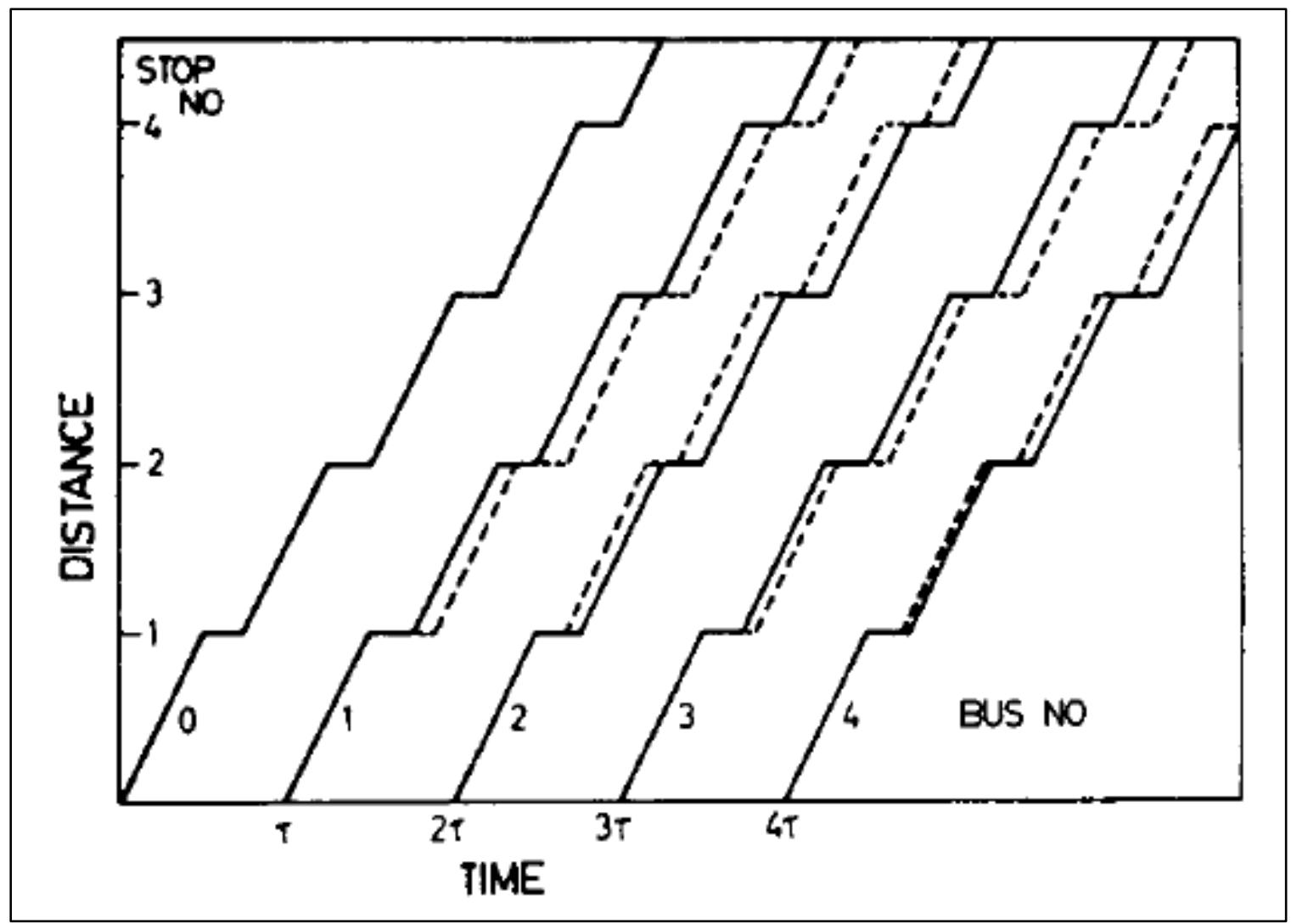

Figure 3: Maintaining a Bus Schedule (Newell, 1995)

Headway is a vital performance measure which needs to be monitored and maintained throughout the day for each trip and each route (Newell, 1995). Headways between buses are important because the average delay for wait time is approximately half of the headway (Newell \& Potts, 1964). There are headways between buses along the entire route, however, these headways are often only known via BDS data available at bus stops after the bus arrival. Extensive research has been done on arrival headways and 
bus bunching, but this may be improved with the knowledge of headways between the stops using 5-sceond high resolution data.

There are several compensating factors to offset bus bunching by bus operators seeing the bus ahead and staying at a stop for a bit longer, a bus running behind may fill up in the peak period and gain time, clocking in at a check point, and possibly bus passing. The theory also suggests that bus bunching can be minimized by keeping the mathematical model ratio, mentioned above, small, which translates to rapid loading. Experiments in Adelaide, Australia have shown that bus bunching does occur in this manner. While deterministic, Hans et al. (2015) developed several stochastic models for a more realistic representation of bus bunching. Alighting was not considered for this model. The models developed forecasted headways for the current bus and the surrounding buses as shown in Figure 4.

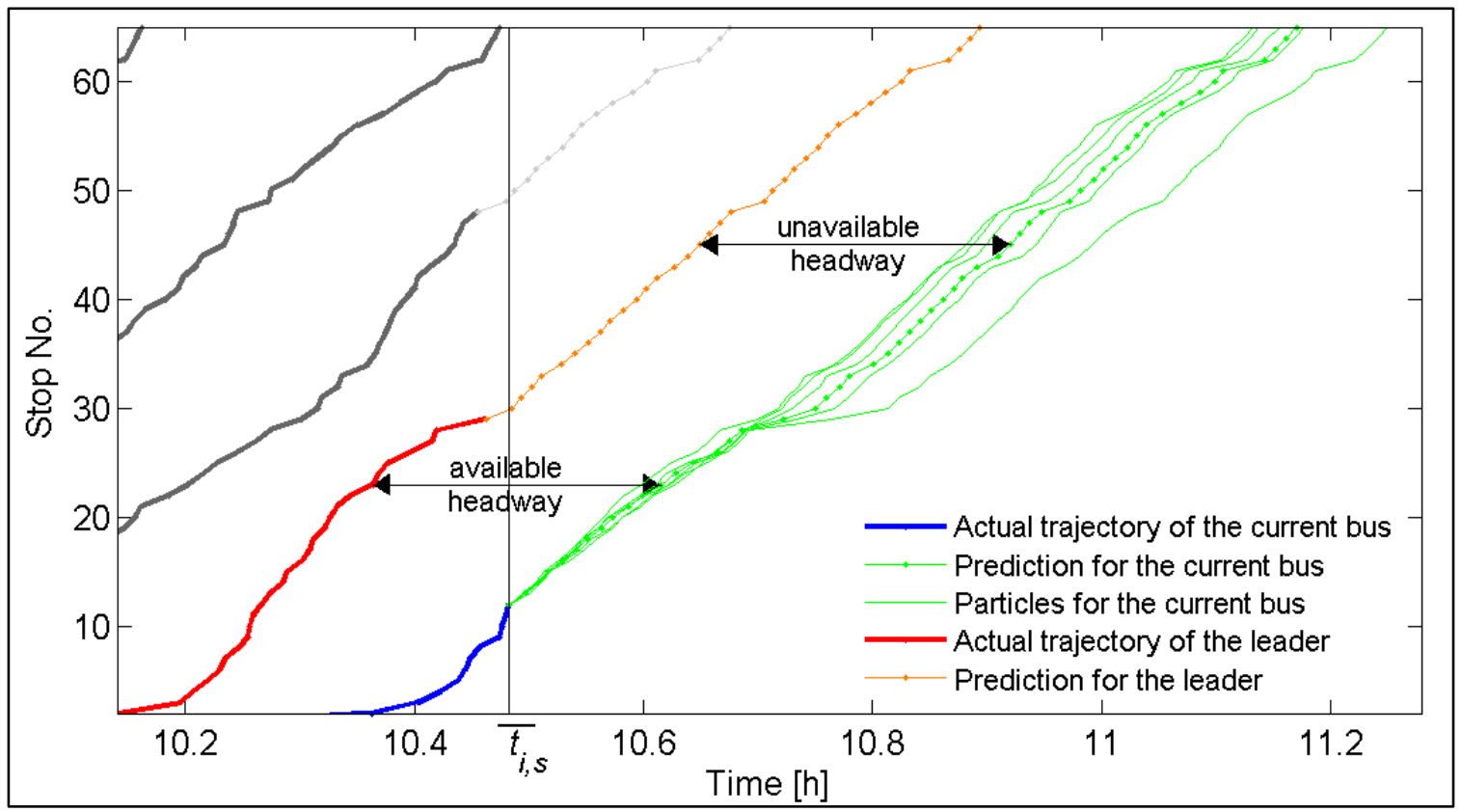

Figure 4: Forecasted Trajectories using Particle Filter (Hans et al., 2015)

Factors such as these can be controlled and with the knowledge of headways between buses it can possibly be prevented. With higher resolution data, headways can be 
predicted between stops and transit operators can be alerted before a bus bunching incident occurs.

Transit agencies have implemented strategies such as transit signal priority (TSP) to reduce transit travel time, reduce travel time and headway variability and improve service reliability. Several studies have investigated the impact of signalized intersections on bus travel time. All TriMet bus routes operate on corridors that utilize TSP. Regression analysis showed TSP to be a significant factor in determining the travel time of the corridor. However, the stop- and intersection-level analyses resulted in TSP effectiveness hidden or evened out at the route level. (Albright \& Figliozzi, 2012). Additionally the impact of adaptive traffic signal control was studied using Sydney Coordinated Adaptive Traffic System (SCATS) along with TSP. TSP was not affected by SCATS, which means there seems to be no additional benefit of TSP to transit vehicles by having SCATS implemented. This study determined the improvements available through SCATS varied at different time of day and in different travel directions (Slavin et al., 2013). Previous studies only estimated the average signal delay due to each additional intersection, and intersection signal timing characteristics were not considered. Stop level data from TriMet and SCATS signal phase log data and intersection vehicle count data were used to study the joint impact of these factors and improvement strategies on bus travel time at the stop to stop segment level. Data integration is important because it provides all the required information for the bus travel time modeling and TSP performance analyses. It is also a challenging step because the bus AVL/APC data and SCATS data are collected in different spatial and temporal dimensions. Bus AVL/APC data are collected at the bus stop level while SCATS data are collected at intersections. 
Results indicated some potential reliability problems of traffic signal controllers receiving and clearing TSP request calls. Furthermore, recommendations included adjusting the settings for several intersections to be more efficient and reducing unnecessary green extension phases (Feng, 2014).

\subsubsection{Dwell Time}

Dwell time is an important parameter that affects transit service quality (Levinson 1983). Dwell time is defined as "the time in seconds that a transit vehicles is stopped for the purpose of serving passengers. It includes the total passenger service time plus the time needed to open and close doors" (HCM, 1985). Dwell time is only recorded if the bus stops and opens its doors; in practice a bus does not stop unless a passenger wants to board or alight the bus (Robinson, 2013). Dwell time is determined mainly by passenger activity at each stop (Chien et al., 2000). The peak periods typically reveal an increase in total dwell time, and a decrease in total dwell time during the off-peak periods (Dueker et al., 2004).

Bertini and El-Geneidy (2004) modeled dwell time for a single inbound radial route in the morning period in their analysis of trip level running time. The results of the dwell time analysis were directly incorporated into the trip time model by estimating parameters for number of dwells and number of boarding and alighting passengers. The mean boarding and alighting times per passenger were found to be 4.2 and 2.1 seconds, respectively, as proposed by Koffman (1978). This theory was proven to be a solid estimate as shown for Route 14 from TriMet by Bertini and El-Geneidy. This paper provides firsthand evidence of the impact of the number of stops and the number of passengers in the estimate of total trip time. In addition to the impacts of dwell time on 
the total trip time, inter-stop time is also a major factor. Figure 5 represents the split between non dwell time (inter-stop) and dwell time (at the stop) (Newell, 1995).

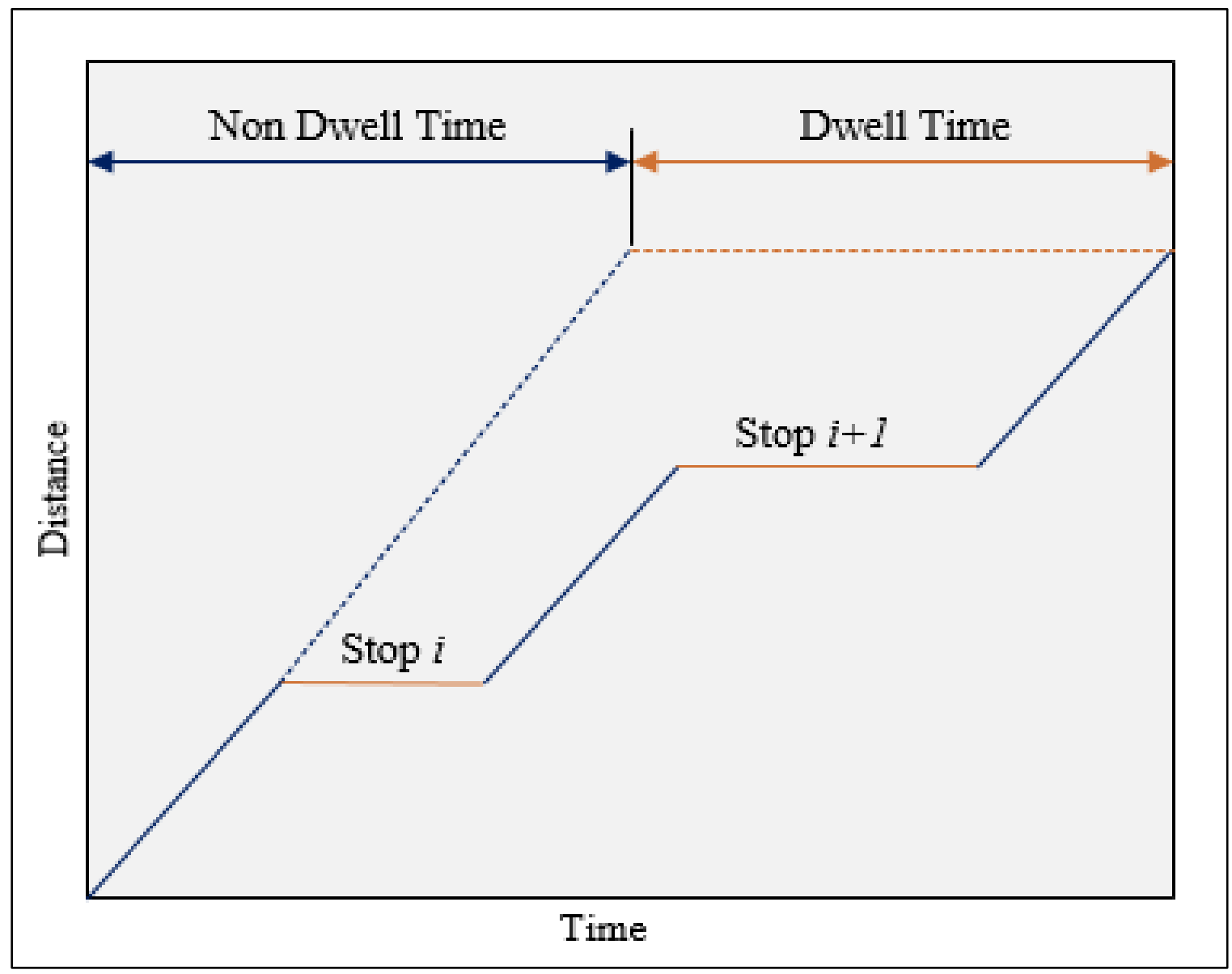

Figure 5: Diagram of Non Dwell Time and Dwell Time Split

The dwell times at all of the stops and the inter-stop times in between all of the stops were decomposed. The trip time model was created using both of these entities separately.

Dueker et al. (2004) modeled dwell time for different times of day, route types and various levels of passenger boardings and alightings. Archived transit data was used to better understand the determinants of dwells, including analysis of rare events, such as lift operations. The mean dwell time was 12.2 seconds with 13.5 seconds of standard deviation, along with an average of 1.2 boardings and 1.3 alightings per dwell. The sub- 
sample with lift operations had a significantly higher mean dwell time and standard deviation of 80.7 and 37.4 seconds, respectively. Boarding and alighting times were analyzed to examine the benefits of low floor buses, which provide savings to the trip time. Dwell time at bus bays possess a high degree of uncertainty originating from the merging behavior of the bus to the vehicles in the shoulder lane. Novel probabilistic methodologies can be used to estimate the dwell time and total trip time (Meng \& $\mathrm{Qu}$, 2013).

\subsubsection{Trip Time Models}

Trip time models can assist transit agencies and passengers. Trip time models can be used to improve reliability. As mentioned, Bertini and El-Geneidy developed a model to predict trip times (2004). Studies find that transit users prefer to reduce travel time variance more than they want reduced average travel times (Gayah et al., 2016). As long as users know that the window for travel time is minimized, then they can have consistencies in their travel on a daily basis. However, transit times vary because of inherent instabilities within bus transit systems that cause buses to naturally bunch or pair when traveling along a route.

In order to predict arrival times of buses a particle filter can be applied. This provides sets of possible bus trajectories which enables the anticipation of irregularities between buses (Hans et al., 2015). These bus models were proposed depending on dwell and inter-stop running time representations which will be replicated in this paper. This study was also performed on TriMet data. Figure 6 illustrates two of the four models developed using simplified approaches. 

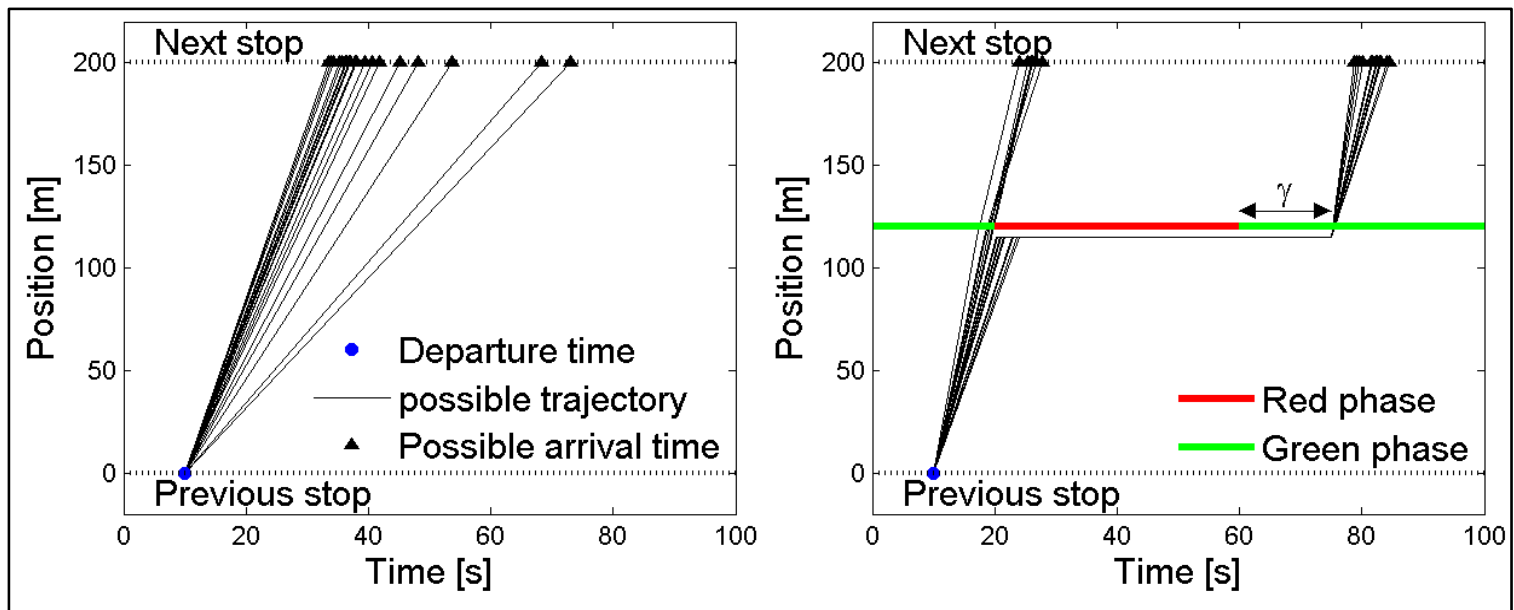

Figure 6: Trip Time Model 1 (Left) and 2 (Right)

The graph on the left predicts the travel time to the next stop using varying trajectories. The possible trajectories are based on 'random events' occurring between stops which range 0-60 seconds. The graph on the right represents trip time model 2 which integrates signal locations and phase settings between stops. However, a different bus model, which accounted for loading provided better forecasts, especially for headway variations. Traffic signal timings and actual traffic flows did not significantly improve the predictions. The lack of uniformity in headways leads to an increase in the mean passenger wait time in addition to longer and more uncertain travel times. Solutions to prevent bus bunching include skipping stops, boarding limits, adding slack time, holding buses at control points, controlling bus speeds, and implementing traffic signal priority. However, if all of these solutions were implemented the efficiency of the transit service could be reduced (Hans et al., 2015).

Similar to previous research, Hans et al. (2015) recommended the use of passenger movements as dependent variables for the dwell time model. Traffic signal settings and traffic flows were used for one of the trip time models, however, these did not show significant impact on the forecast duration compared to the probabilistic 
distributions of less data intensive trip time models. The methods described were very efficient for anticipating headway variations. The framework from this paper proposed smoother strategies to prevent bus bunching. Similar dwell and trip time models were considered for this thesis. Possible future studies could be done to improve the prediction particle filter by using higher resolution data in addition to automatic vehicle location data. There might be a point where higher resolution data can provide the more information and predict better trajectories.

\subsubsection{5-Second Resolution Data}

Dwell time, passenger movement, and total trip time analyses are achievable through the use of stop-level BDS data. However, this dataset is insufficient for segment, inter-stop, real speed and acceleration analyses. Stop-level AVL data provides valuable information at the stop, but everything that happens between the stop is unknown. In their earlier system, TriMet stored the maximum speed achieved between stops but nothing else. Applications of second generation archived transit data for estimating performance measures and arterial travel speeds were initially determined with similar 5 second resolution (5SR) data (Glick et al., 2015). Finer resolution bus travel speeds as a means to examine speed changes, queuing and delay at signalized intersections and other locations along the route were examined. Analyses of average speeds created using 5SR data at each intersection can indicate where buses are stopping and highlight whether those locations are intended to be slow moving or a stop as shown in Figure 7. 


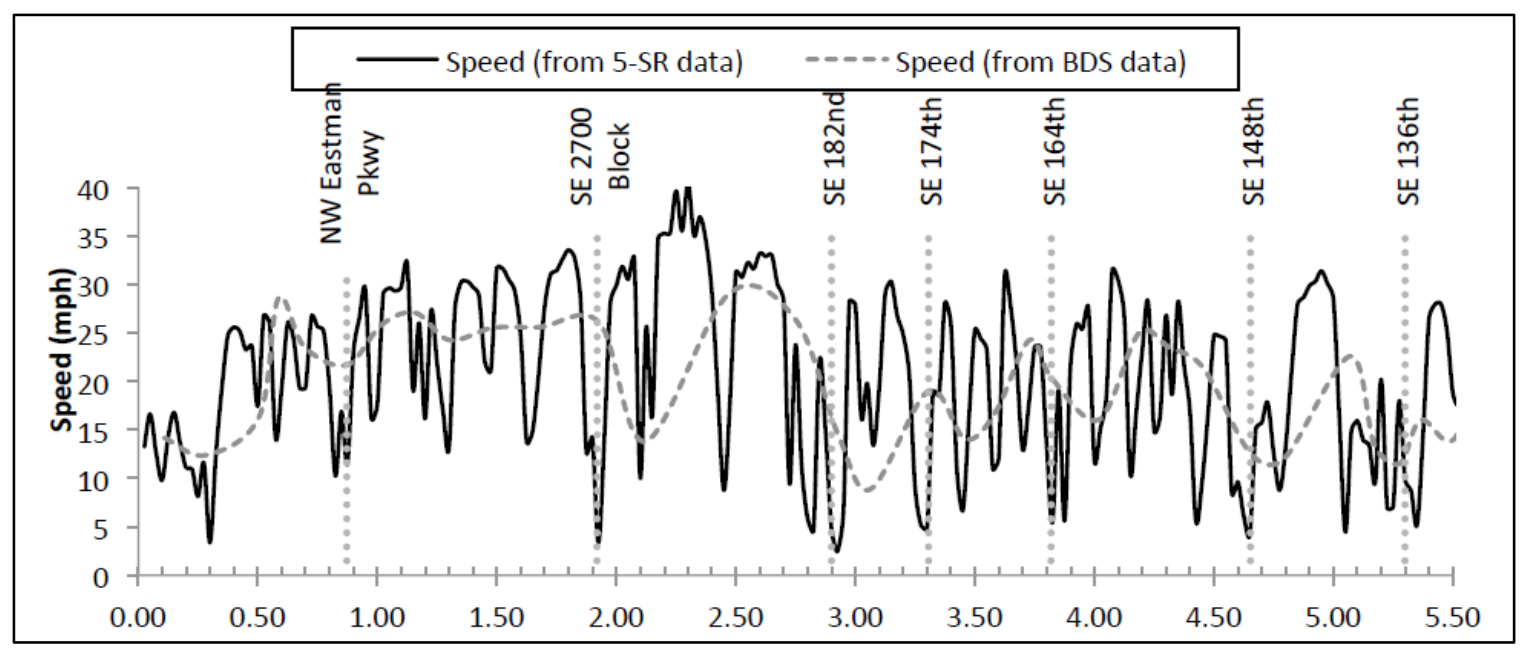

Figure 7: Speed Comparison of 5SR vs BDS (Glick et al., 2015)

This speed plot shown in Figure 7 was created for the AM peak period, 6-10 AM for one day. The speed breakdown from TriMet's Route 9 showed that approximately $46 \%$ of the time was spend moving below $5 \mathrm{mph}$. Additionally, similar 5 second data was used to discover trends and travel patterns along urban arterials with only a few days' worth of data. The purpose was to improve bus running times/reliability and develop arterial performance measures. Congestion and speed variation can be viewed by time of day and plots can help indicate delays caused by intersections, crosswalks or bus stops. Buses were used as travel probes, and contoured speed plots were created for the behaviors of roadways outside the zone of influence of bus stops, as shown in Figure 8 (Stoll et al., 2016). 


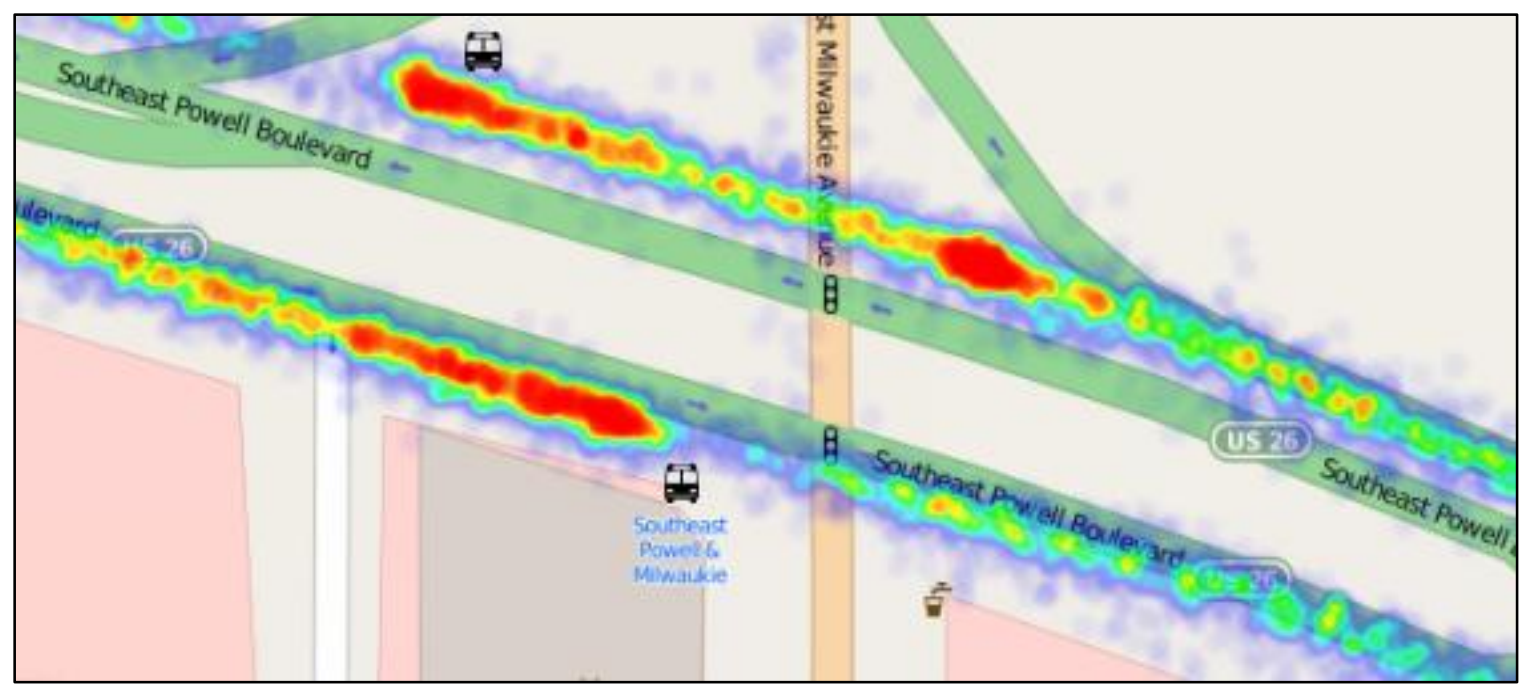

Figure 8: Heat Map of Speeds on Powell Blvd using 5SR (Stoll et al., 2016)

Results from these studies suggest that the new generation of higher resolution bus trajectory data can be successfully employed to observe and identify metrics in more detail than could previously be seen by using stop level AVL data. The high resolution data allowed researchers to determine bus travel speeds between bus stops, categorize speed breakdowns, and identify intersection signal/queuing delays. This higher resolution data removed the need for some educated guesswork when using buses as probes or determining performance metrics between stops. No conclusions were drawn about the intersection examined other than a statement that the data could be used for such an analysis; therefore, a gap in research still exists (Stoll et al., 2016).

Probe vehicles are often used as an effective means for obtaining arterial travel times (Bertini \& Tantiyanugulchai, 2004). Transit buses experience travel times that are inherently different from those experience by the private vehicles due to a number of factors associated with servicing passengers at bus stops and we refer to these factors as bus biases. The bus stop creates a bias at the bus stop location in three ways: the time spent at the bus stop, the time lost decelerating to and accelerating from the stop, 
additional waiting time at an adjacent traffic signal after door closure, and any merging delay while the bus is waiting for an acceptable gap to reenter the traffic stream, switching lanes, time point idling and different vehicle dynamics of cars and buses.

With the deployment of AVL systems to monitor transit vehicles (and other fleet vehicles), there have been several efforts in recent years that attempt to obtain arterial travel times for the private vehicle population. The fleet vehicle data capture valuable information, such as the traffic conditions, but they also capture servicing passengers at bus stops. There are several strategies used in conventional practice to eliminate the biases that occur in the vicinity of the bus stop.

The use of buses as probes to estimate travel times has been well studied in the past. However, these studies used stop-level data, which was all that was available at the time. Proxies have been used to estimate travel times and trajectories between stops. The estimated stop time, which does not account for bus accelerations or decelerations, was removed and the recorded/reported maximum speed in between the stops was used to estimate the travel time (Glick, 2015).

This research uncovered a fundamental issue impacting almost all systems that use buses as arterial travel time probes: even when the transit operation times are completely accounted for at the bus stops, large biases relative to the private vehicles remain. Even after removing the transit related delays of servicing passengers at a stop, the delay will knock the bus out of progression and thus, it will encounter different delays than the private vehicles when reaching signals downstream. The progression errors at subsequent traffic signals far downstream of the bus stop can be much larger than the local impacts at the bus stop. The net impact could be positive or negative. In short, using 
data from a single run, the transit buses as probes cannot correct for the progression errors that occur far away from the bus stop location. The work was limited to the fairly short corridor in the dataset and the paper simulated a single bus stop (Glick, 2015). In a corridor with many bus stops the impacts could be much larger as the different stops interact with the signalized intersections. Across successive intersections the combined transit operations and progression impacts could compound in to large biases or they may cancel out.

The interaction between transit vehicles and signalized intersections is an important factor that must be accounted for, one cannot determine how this factor will impact the travel time estimates without studying the interactions unique to the given corridor. This caution should also extend to the use of any fleet of vehicles as probes if the given fleet behavior differs from the private automobiles, such as, taxis and public service vehicles, may exhibit biases against the private vehicle population. Furthermore, other potential bus biases include travel time differences across lanes, time point idling, and the different vehicle dynamics of cars and buses (Thornton \& Coifman, 2014). Although not explored in this thesis, transit signal progression and the use of buses as probes may be improved with the higher resolution data and ability to remove loss time and dwell time due to bus stops.

\subsubsection{Summary of Literature Review}

Extensive research has been done on public transportation using stop-level BDS data. The AVL and APC systems provide valuable information that can be used retrospectively to analyze bus trajectories, impacted bus stops, bus bunching, benefits of transit signal priority and more. Newell split the trip time model into dwell time and 
inter-stop time (1964). The previous trip time model (Bertini \& El-Geneidy, 2004) focused more on the dwell time portion and used a simplified approach on the inter-stop portion of the model using BDS data. Results from (Glick et al., 2015) and (Stoll et al., 2016) suggest that the new generation of higher resolution bus trajectory data can be successfully employed to observe and identify metrics in more detail than could previously be seen by using stop level AVL data. This higher resolution data will be used alongside stop level data to improve the trip time model and explore useful applications of this data. 


\section{DATA}

The data used for this thesis was retrieved from the Tri-County Metropolitan District of Oregon (TriMet). TriMet is one of the first public agencies to try to tackle the problem of online transit trip planners though the use of open datasets shared with the general public. TriMet worked with Google to provide quality transit schedule, route and stop data in an electronic format that would constantly be up-to-date. This transit data format is now known as the Google Transit Feed Specification (GTFS). TriMet is revolutionary and one of the top public transit agencies in the nation.

TriMet provides a robust amount of real-time information in-house along with full developer support. The in-house services include Transit Tracker website, mobile website, SMS and automated phone response all provided by TriMet. Quick Response (QR) codes are provided at all stops. Developer coordination includes TriMet App Center, GTFS-real-time feed, Google Support page, and contacting an employee within the GIS/Location Services department at TriMet. The error monitoring includes posting to the TriMet Support page.

\subsection{Study Route}

The route chosen for this study was a 7.2 mile segment of TriMet's Route 14 inbound (west-bound), shown in Figure 9. Route 14 is listed as a "frequent service" bus line because it runs every 15 minutes or better most of the day, every day. This route is a heavily used inbound route that runs through southeast Portland toward the downtown center during the morning commute period. TriMet provides 64 scheduled trips per weekday on Route 14, with 45 stops for the inbound (towards downtown Portland) trip. Scheduled trip times range between 35 and $45 \mathrm{~min}$ (mean trip time of $40.2 \mathrm{~min}$ ) and 
scheduled headways range between 5 and 55 min (mean headway of $13.8 \mathrm{~min}$ ). The analyses here concentrate on the inbound service between SE 94th/Foster Ave. (location ID 1831) and the North Terminal, a layover and bus staging area at SW Madison \& 6th (location ID 3639).

\section{4-Hawthorne}

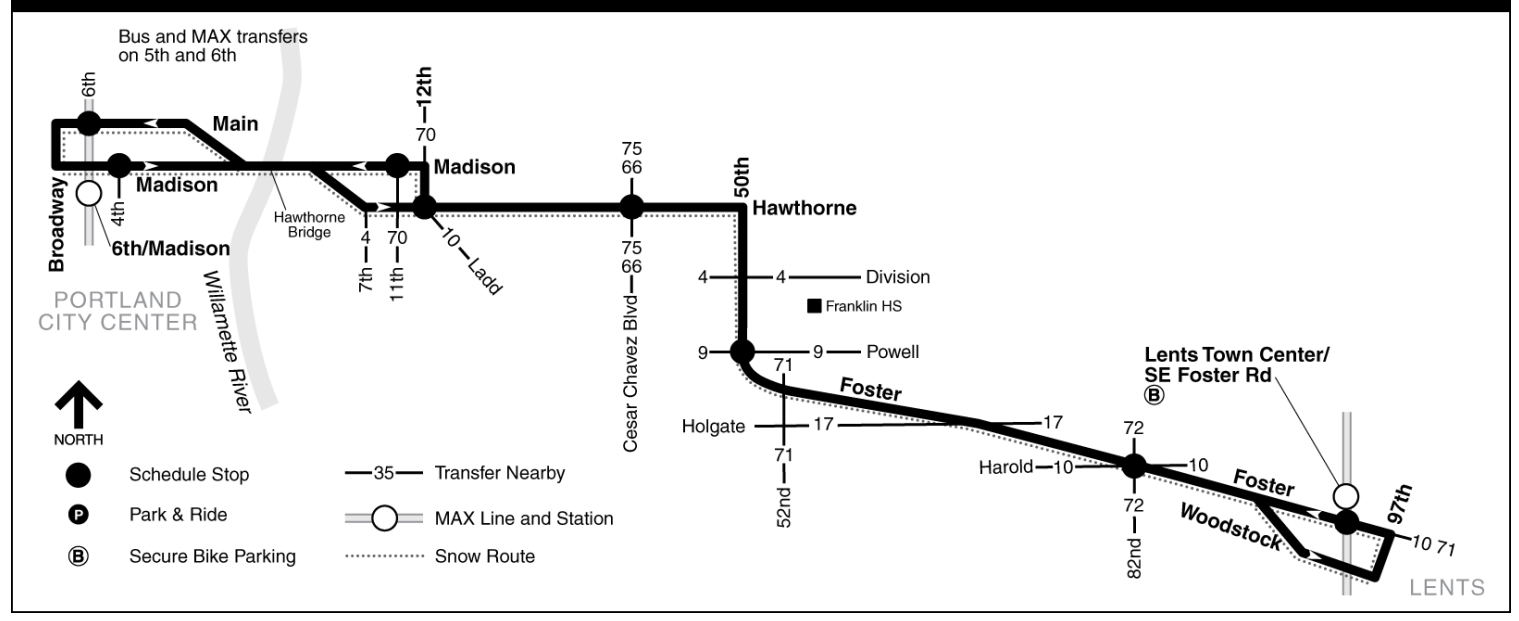

Figure 9: Study Route 14 (TriMet, 2016)

The first trip on Monday, October $6^{\text {th }}, 2014$, starts at 4:56 AM at SE Foster \& 94th and goes to the Portland City Center, ending at 5:26 AM at SW Main \& 6th. The last trip of a weekday starts at 1:05 AM and ends at 1:34 AM. TriMet changes the schedule times quarterly. The average trip time is higher than these two trips because there is more traffic congestion and ridership at peak periods. In order to demonstrate and compare the modeling approaches, 72 trips were used for the inbound route with 45 stops. Additionally, 14 inbound trips between $6 \mathrm{AM}$ and $10 \mathrm{AM}$, morning peak period, for October 7-9 and 14-16, 2014 were analyzed. The next section contains a description of the study corridor and the two sources of data used in this study.

\subsubsection{Study Corridor}

Route 14 begins at the Interstate 205 interchange onto SE Foster Road. The route 
continues on SE Foster Road at an angle with a posted speed limit of $35 \mathrm{mph}$ until SE Powell Blvd. Figure 10 illustrates the path of Route 14, southeast suburbs to the downtown center, with signalized intersections denoted with signal head symbols.

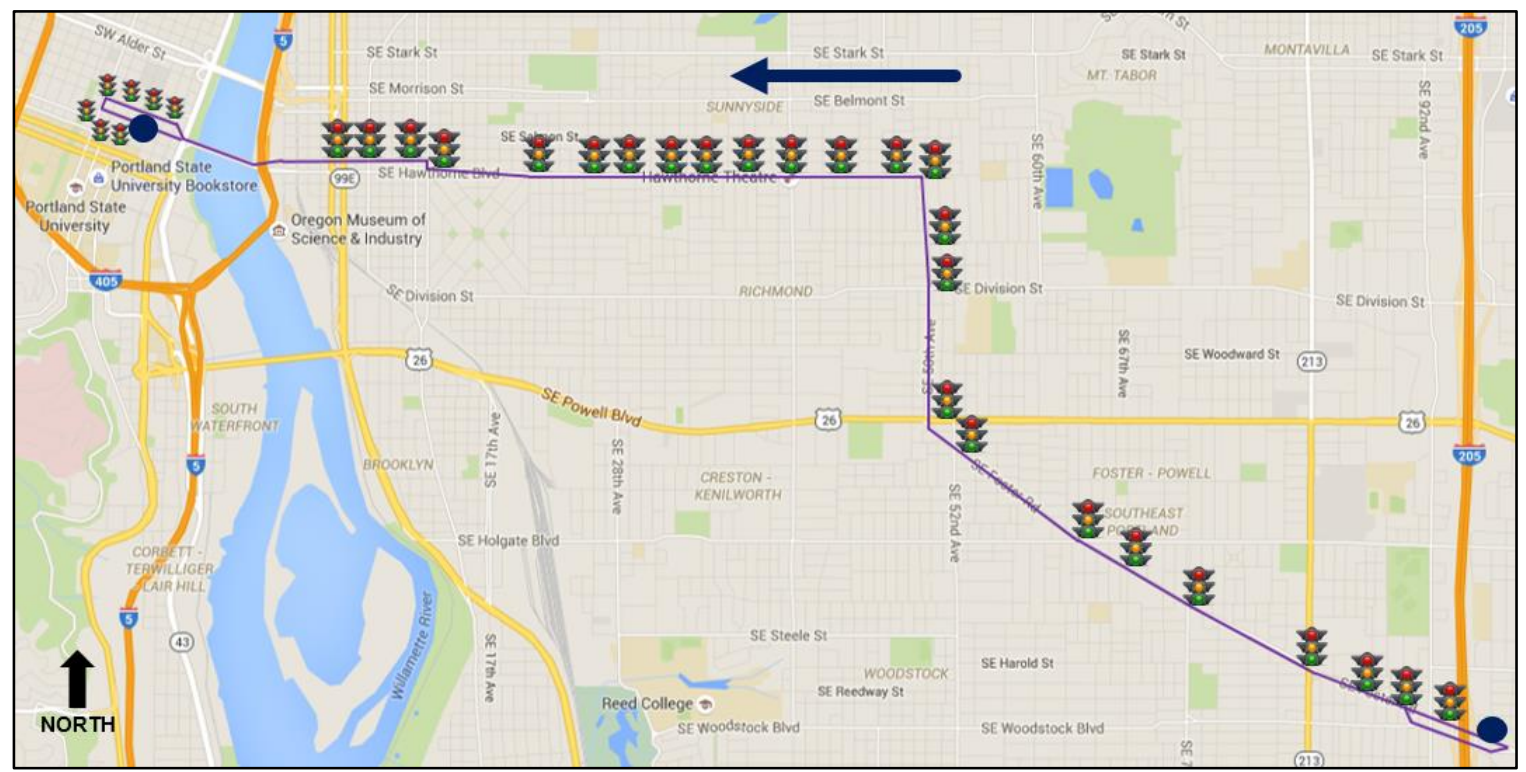

Figure 10: Google Maps Photo of Route 14 with Signalized Intersections

There are 32 signalized intersections, excluding pedestrian crossing signals. Once the bus is downtown, there is a signalized intersection every block. The route crosses SE 82nd Ave, also known as OR213, a state highway. Additionally, SE Powell Blvd is also known as US 26, a major arterial which causes delays for the 'minor' street at the signalized intersection. After crossing SE Powell Blvd onto SE 50th Ave, the route takes a left onto Hawthorne Blvd for the longest stretch, at a posted speed limit of $25 \mathrm{mph}$. The focus of this study will be the inbound trips, which correlate to the westbound direction on Hawthorne Blvd. The Portland Bureau of Transportation reported this arterial has an average daily traffic volume of 8,400 and an AM peak hour volume of 880 (2014).

There are several vehicles which traverse this route, several vehicles are on the cross streets as well. Each segment varies by distance and what is between them from 
stop to stop. Table 1 describes how many segments have no signals, pedestrian signals, and if signalized is the stop a near-side or far-side.

Table 1: Number of Signalized/Unsignalized Segments along Route 14

\begin{tabular}{|l|c|}
\hline \multicolumn{1}{|c|}{ Type of Segment } & Count \\
\hline \hline Pedestrian Signal & 3 \\
No Signal & 19 \\
Signal & 26 \\
Far Side Bus Stop & 7 \\
Near Side Bus Stop & 19 \\
\hline
\end{tabular}

After traveling on Hawthorne Blvd there is a short turn onto a one-way stretch of SE Madison Street in order to access the Hawthorne Bridge to enter the Portland city center. The Hawthorne Bridge crossing over the Willamette River is a truss bridge with a vertical lift that joins Hawthorne Blvd and Madison St; it is the oldest vertical lift bridge in operation in the United States. It is also the busiest bicycle and transit bridge in Oregon, with 800 TriMet buses carrying about 17,500 riders daily (TriMet, 2016). This bridge has one lane in each direction and since there is a constant flow over the bridge (except when the bridge is lifted), the analysis will be performed before it reaches this point. As mentioned in the literature review, all of the signalized intersections along the route have transit signal priority, but there are some difficulties receiving and granting the signal (Feng, 2014).

\subsection{Bus Dispatch System (BDS) Data}

Currently, TriMet operates 97 bus routes and a 38-mile light rail line within the tricounty Portland metropolitan region. The bus lines carry approximately 200,000 trips per day, serving a total population of 1.5 million persons within an area of 590 sq. miles (TriMet 2016). TriMet has implemented a unique Bus Dispatch System (BDS) that 
collects stop-level data as a part of their overall service control and management system (Strathman et al., 2001). The main components of the BDS include

- Automatic Vehicle Location (AVL) using a satellite-based Global Positioning System (GPS).

- Voice and data communications via cellular and radio

- On-board computer and control head displaying schedule adherence to operations, detection and reporting of schedule and route deviations to dispatchers, and twoway pre-programmed messaging between operator and dispatchers.

- Automatic Passenger Counters (APCs) on front and rear doors of all vehicles in the bus fleet

- Dispatch center with computer aided dispatch (CAD)

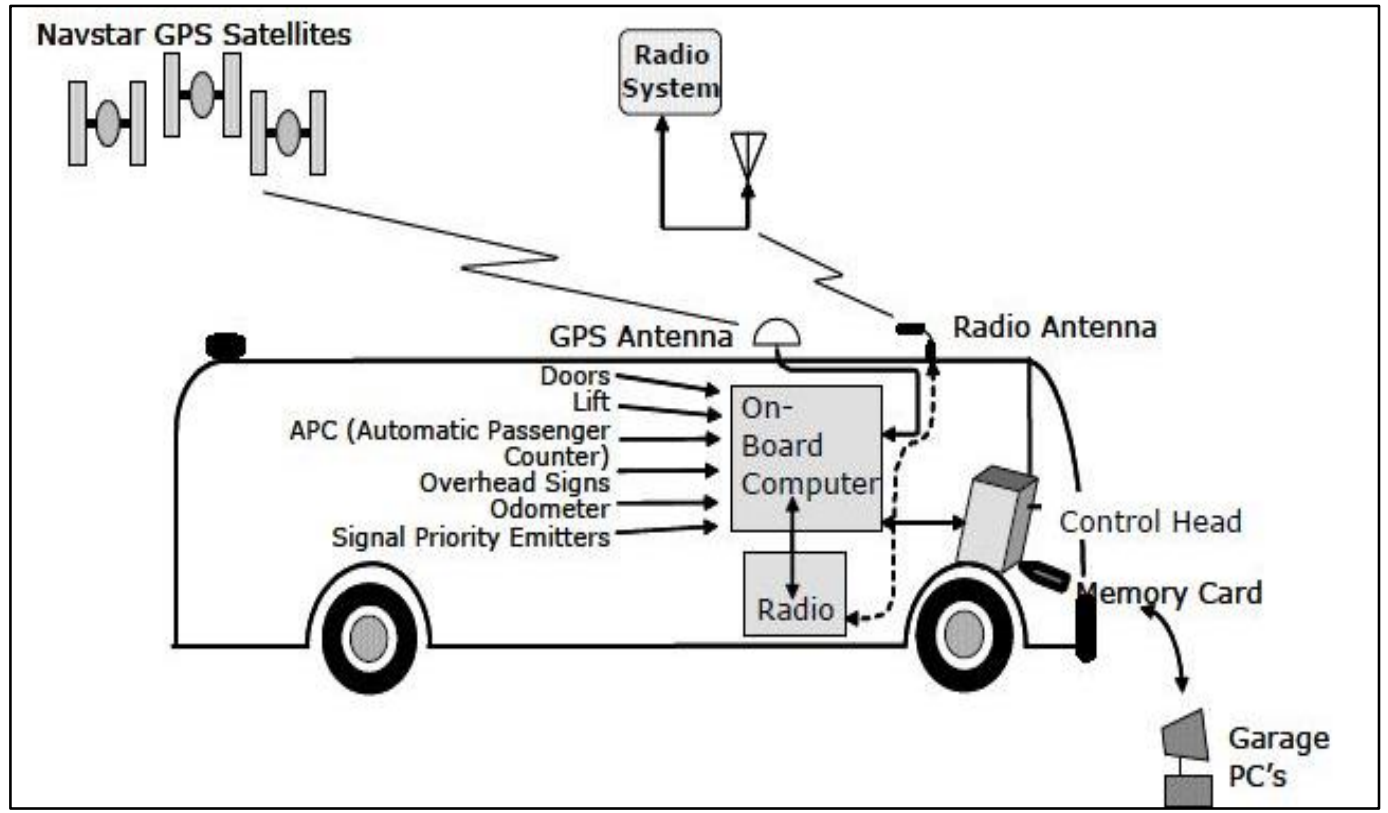

Figure 11: Components of BDS Data (Tantiyanugulchai, 2004)

The BDS system is comprised of three main components: the GPS satellite system, the real time information system and the data archive system. The GPS satellite system provides vehicle location information feeding into the AVL system in order to monitor 
vehicle locations in real time. The on-board computer determines the vehicle location and transmits the real time information to the transit dispatch center. The real time communication system also supports voice and data communication using a mobile radio system. The dispatch center receives the information from the vehicle at a regular interval, or in response to an event such as a detour, accident or vehicle breakdown. This system is used to ensure that the bus dispatch center is updated with at least the minimum amount of information for tracking and reporting purposes and to provide assistance to bus operators. The most important part of the BDS that is useful for this study is the archived component. As shown in Figure 11, information regarding bus operational characteristics such as distance traveled, passenger activities, vehicle location (GPS coordinates) and maximum speed achieved on every link traveled, are recorded into a storage memory card while the bus is in service. At the end of the day, the archived data is downloaded to the control system (Tantiyanugulchai, 2004).

The arrival time and departure time are collected in total seconds. The number of boardings, alightings and the estimated load are collected using the automatic passenger counter. Also, the location, in NAD83 state plate $x-y$ coordinates, is collected at each stop. Additionally, the system collects the maximum speed, in miles per hour, achieved between stops. As shown in Figure 12, the data is recorded at each stop, which is geocoded with a predefined 150 -foot stop circle surrounding the stop. 


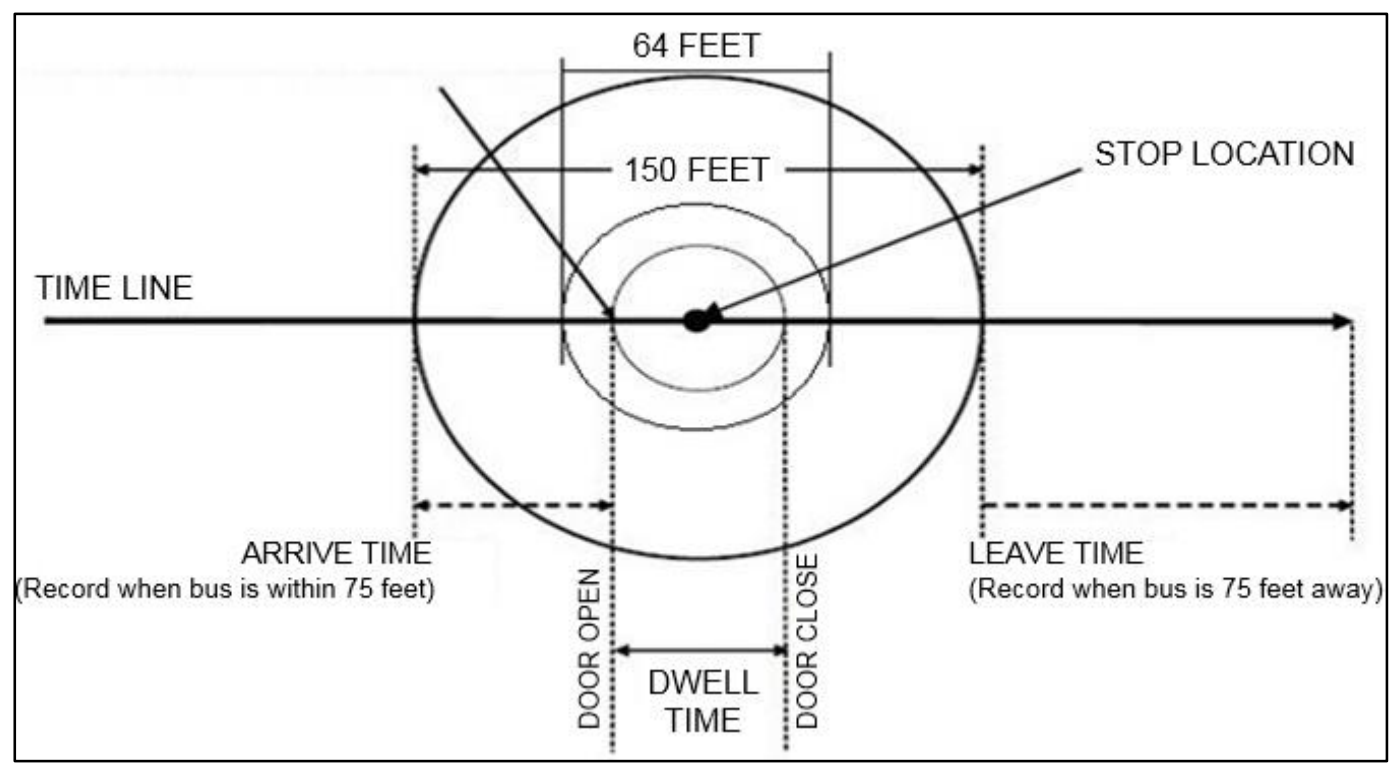

Figure 12: Stop Circle where the BDS Records Times and Locations

If the bus does not stop at the stop location, the BDS records the time that the bus is within 75 feet of the stop. The arrive time and leave time are the same if the bus does not stop at the bus stop. However, if the bus stops at the stop location, the BDS records the arrive time within 32 feet of the stop. Next, the dwell time is recorded when the doors open until the doors close. Once the bus is 32 feet outside of the stop, the leave time is recorded. The same process follows when the bus arrives at a layover location with a large bay size, typically 75 feet. The dwell time is provided by subtracting the arrive time by the previous leave time. Additionally, the scheduled time is also provided for each stop. Furthermore, the passenger movement is also provided at each stop. An example of the BDS output is presented in Figure 13. 


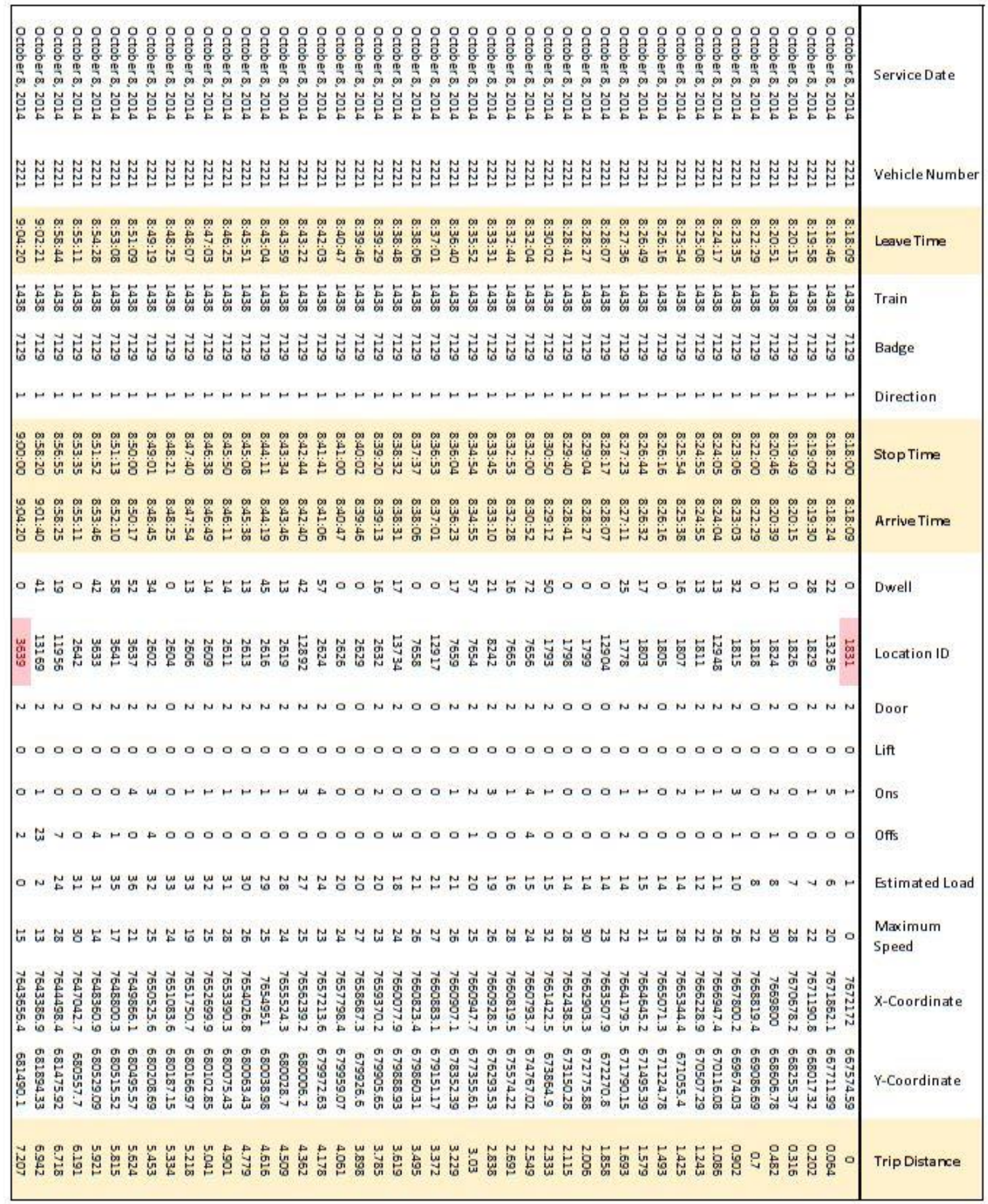

\section{Figure 13: Example of BDS Data}

This sample of data displays one inbound trip, the red cells identify the first and last stop location IDs. The tan colored columns represent calculated/edited values. The times are all recorded as seconds and must be converted into Hour:Minute:Second format. 
Additionally, the trip distance needed to be calculated from the $x-y$ coordinate location information which is described in the next section.

\subsubsection{Data Preparation}

The procedure required to convert the $x-y$ coordinates to trip distance is:

$$
y=\cos ^{-1}\left(\sin \left(\frac{l a t_{1} * \pi}{180^{\circ}}\right) \cdot \sin \left(\frac{l a t_{2} * \pi}{180^{\circ}}\right)+\cos \left(\frac{\text { lat }_{1} * \pi}{180^{\circ}}\right) \cdot \cos \left(\frac{\text { lat }_{2} * \pi}{180^{\circ}}\right) \cdot \cos \left(\frac{\operatorname{long}_{2} * \pi}{180^{\circ}}-\frac{\text { long }_{1} * \pi}{180^{\circ}}\right)\right) \cdot 3959
$$

The $x-y$ coordinates are input into the formula as longitude and latitude, respectively. The end result, as shown in the sample of data above, is cumulative trip distance in miles. Once each of the trip distances was calculated, the stop locations appeared to have inconsistencies. All of the stops are fixed locations and each trip should record the location at the same point. In order to compare the data collected by GPS for the route is accurate, the $x-y$ coordinates were be converted to be compared to the Northings/Eastings of each stop location from the Tri-Met Interactive Map (2016). This is necessary because the GPS typically loses signal in downtown. As shown in Appendix A, the GPS location of the bus is collected within 100 feet of the actual stop. Therefore, several stops are collected at slightly different locations. However, the stops are all located at fixed points and they need to be placed at the same distance across all of the trips for Route 14 . Google Maps was used through the TriMet's online interactive map to establish stop locations for all of the trips. Figure 14 illustrates the stop location comparison between Google Maps and the BDS data collection. This graph shows that both datasets overlap and the Google Maps points are sufficiently accurate to use in this analysis. 


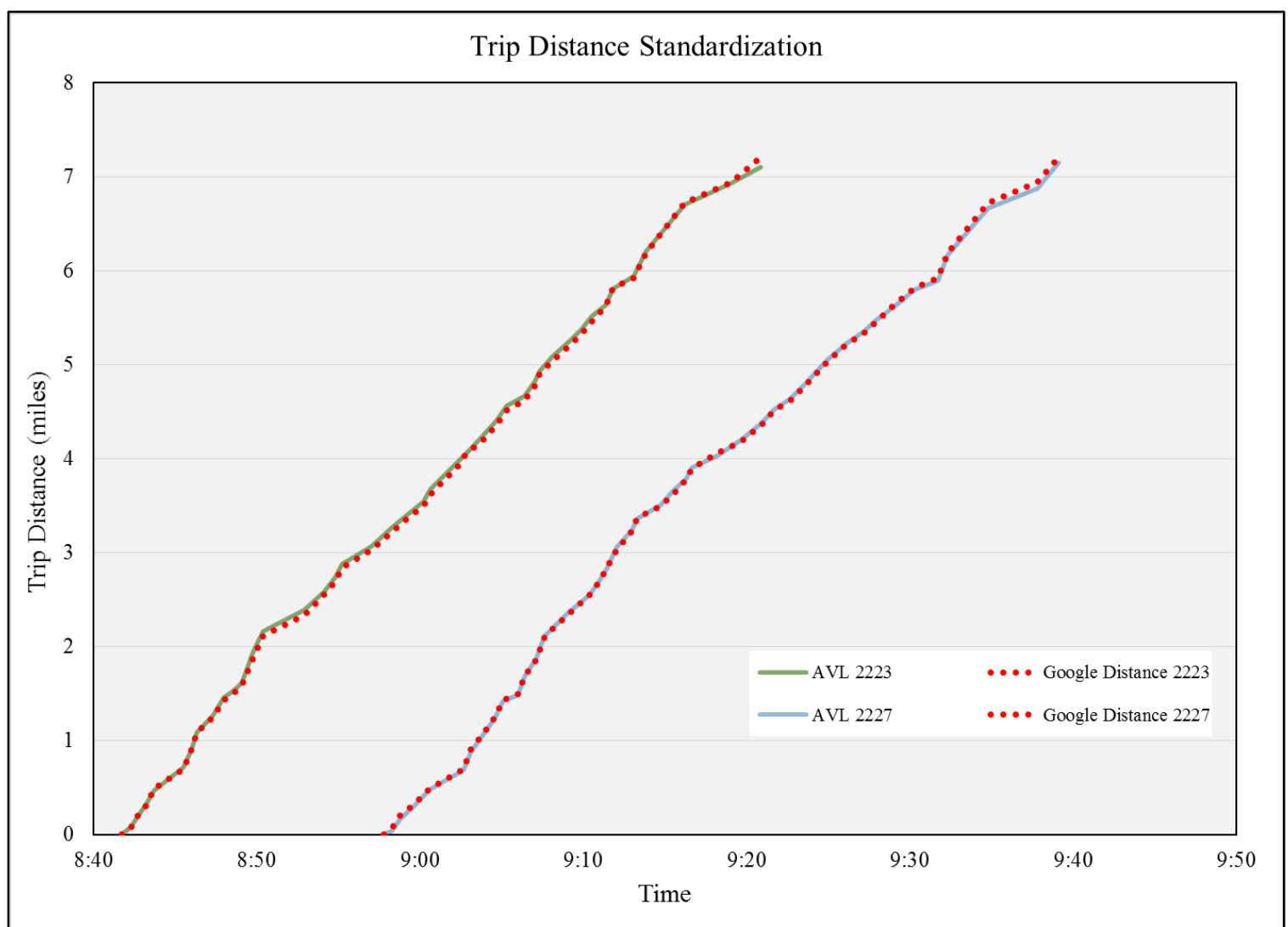

Figure 14: Trip Distance Comparison of BDS and Google Distance

These measured distances were used in place of the Route 14 distances from BDS. Using this method, it still allowed for the unscheduled stops to occur, they just had to be interpolated using the original trip distance by the data. Some routes had to be removed because of missing locations. A time-space diagram of all of the routes shows the trajectories of the trips in Figure 15. The BDS data for Tuesday, October 7, 2014, was used to create trajectories for each trip. Each row includes the leave and arrive time at the stop, this represents the time spent at the stop, dwell time. In order to illustrate dwell time in graphical form the 'arrive' times and 'leave' times are split with the corresponding distance of each stop, these are shown as horizontal lines (same location for a period of time). 


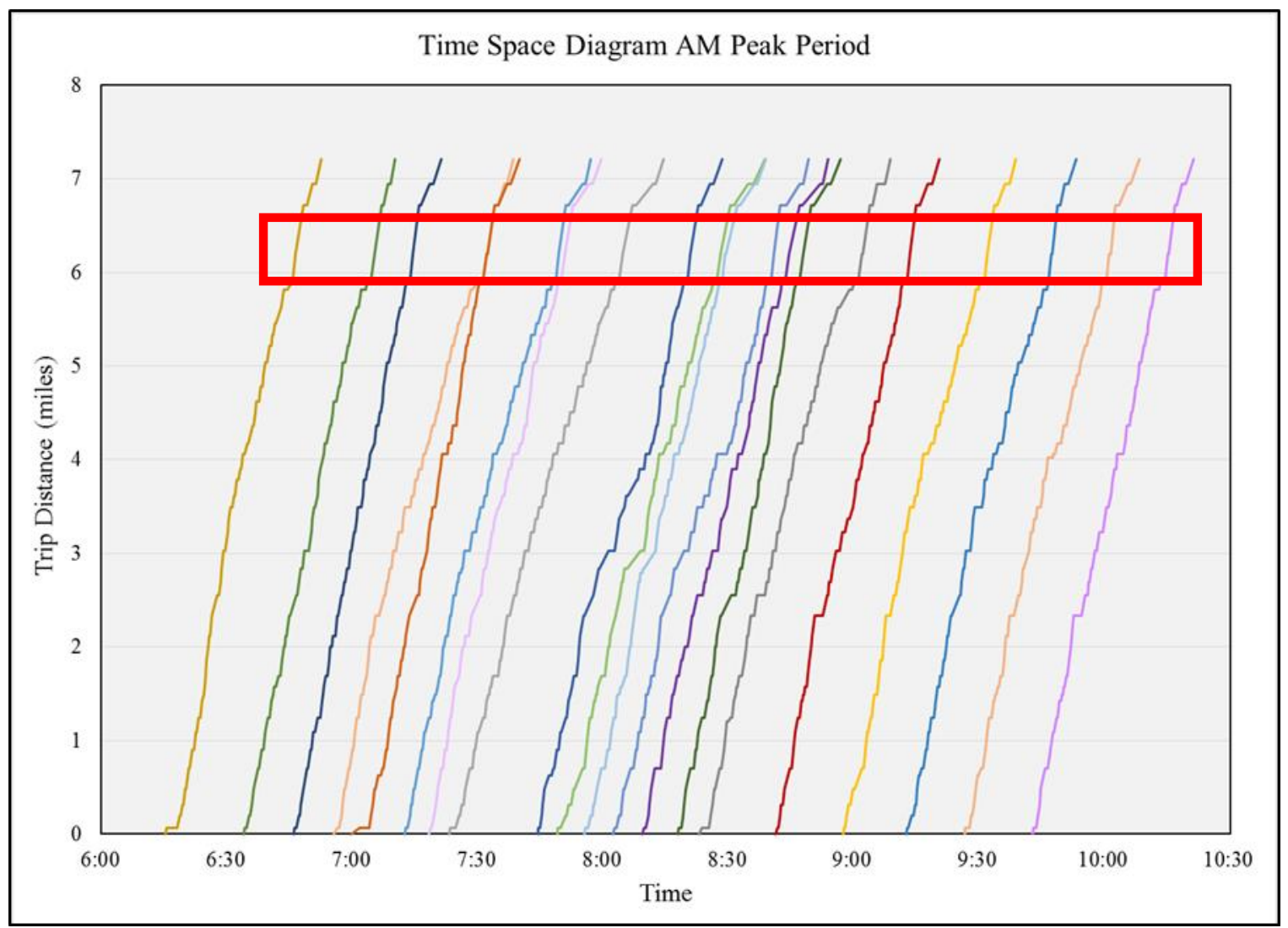

Figure 15: Example of Bus Trajectories on a Time-Space Diagram

The figure shows the constant slopes from mile 6 to 7, this represents the bus traveling the longest distance without any stops over the Hawthorne Bridge. The trajectories allow transit agencies to determine major bus stops (long horizontal lines: dwell time), congested segments (lower speeds) and the presence of bus bunching (lines near each other).

\subsubsection{Passenger Movement Data}

As shown in the sample of the BDS data, the infrared beam automatic passenger counters (APCs) are located on each door and provide detailed information about passenger movements on the bus. In addition to the number of passengers that board and alight at each stop, the estimated load is provided using algorithms. Approximately $0.9 \%$ of stops used lifts for assisting passengers with disabilities in an entire day, compared to 
$0.7 \%$ in 2003 (Bertini \& El-Geneidy, 2004). However, there are instances where this information is missing or inaccurate (outliers), which were removed from analysis. This information is valuable to determine which stops are most impacted and allows transit agencies to develop dwell time models for the entire trip. Figure 16 represents the average estimated loads of the bus for the AM peak period for October 7-9, 2014.

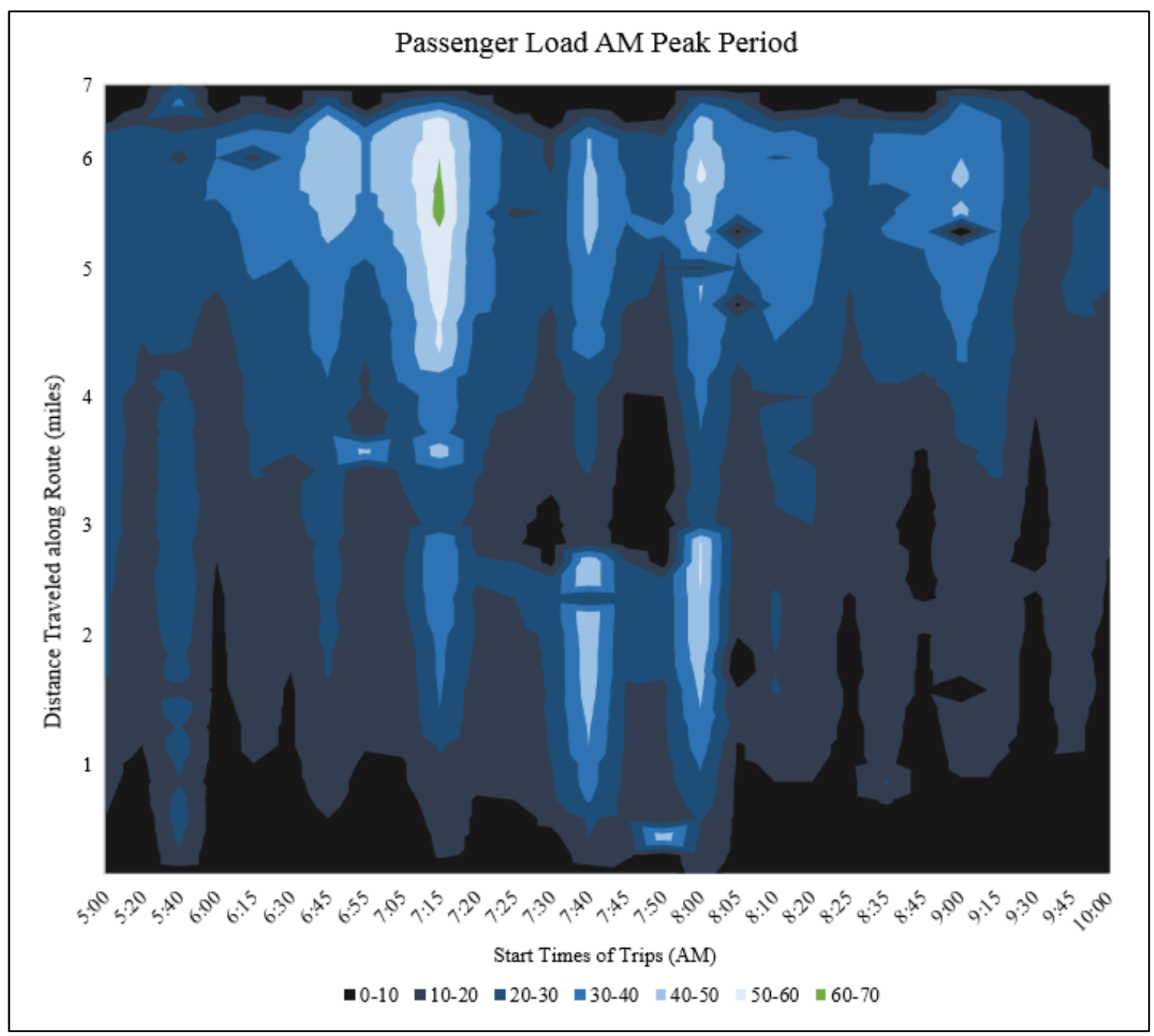

Figure 16: Passenger Load for AM Peak Period using BDS Data

The bus appears to reach full capacity around mile 6 of the trip at 7:15 AM, which is reasonable for the morning commute time. This contour map of the passenger load is developed using the estimated load data provided by BDS. 
Two trips from the AM peak period of Tuesday, October 7, 2014, shown previously in Figure 15, were analyzed further by incorporating the passenger movements (right hand axis) with the time space diagram (left hand axis) shown in Figure 17.

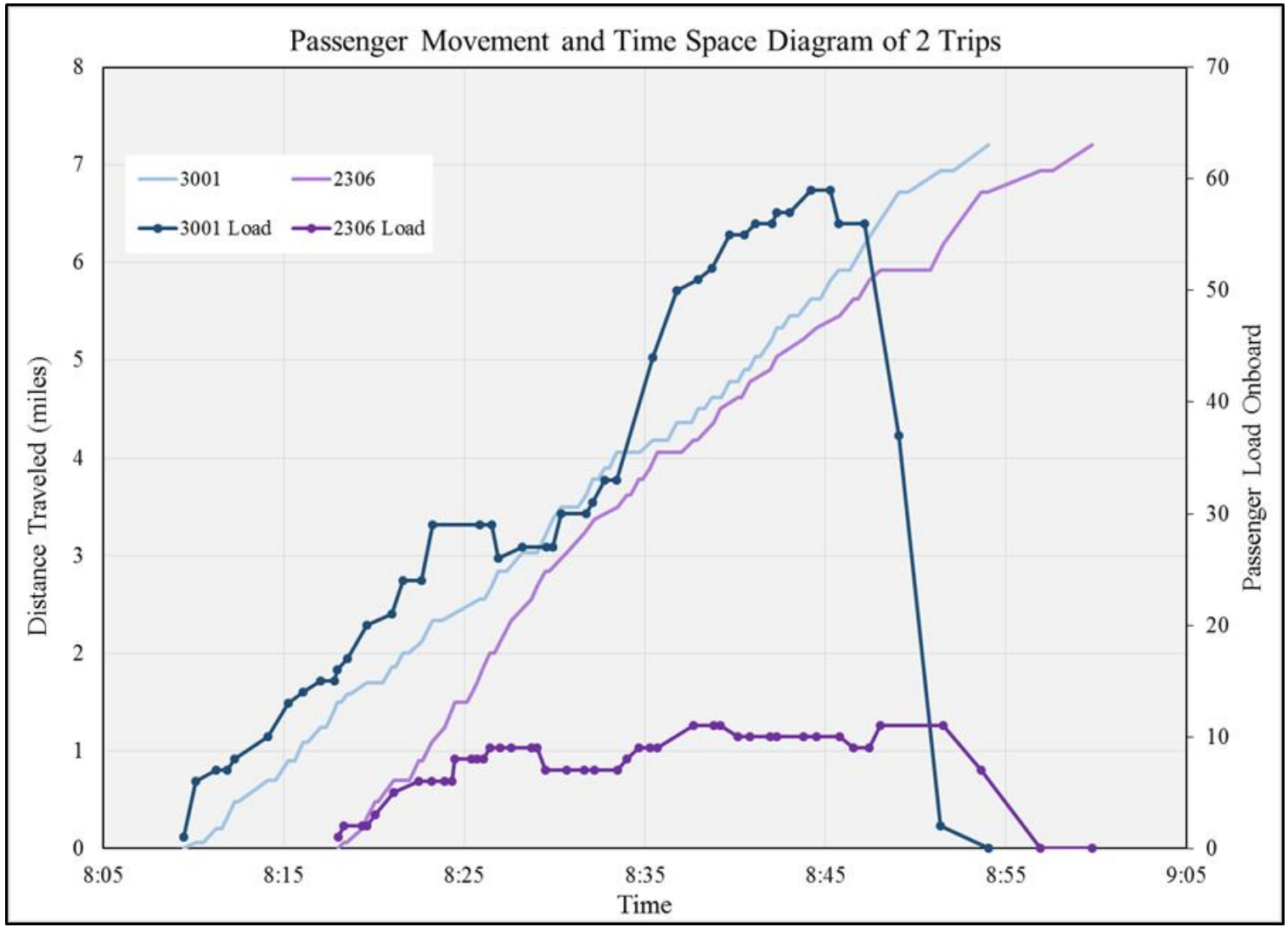

Figure 17: Passenger Movement and Time Space Diagram of 2 Trips

As shown in Figure 17, there is large disparity in the estimated passenger loads of the two buses. The added knowledge of passenger load with the time space diagram follows the same principles from Newell (1995) for bus bunching, illustrated previously in Figure 3. If the first bus becomes late, it picks up more passengers that have arrived later, this causes the bus to continue to get more late as it takes more time to pick up these extra passengers. Alternatively, the second bus will have less passengers to pick up and it will continue to get more early until "bus bunching" occurs. Once this phenomenon occurs, it is important to analyze the headway between buses. 


\subsubsection{Headway Data Preparation}

The BDS data does not include a column with headways between buses, this must be calculated. The arrival time of bus 2 is subtracted by the arrival time of bus 1 to determine the headway between buses at a particular stop. Using the filter command in Excel, the headways were calculated between consecutive buses for every inbound stop. Figure 18 provides a graphical representation of headway between two consecutive trips in the AM peak period.

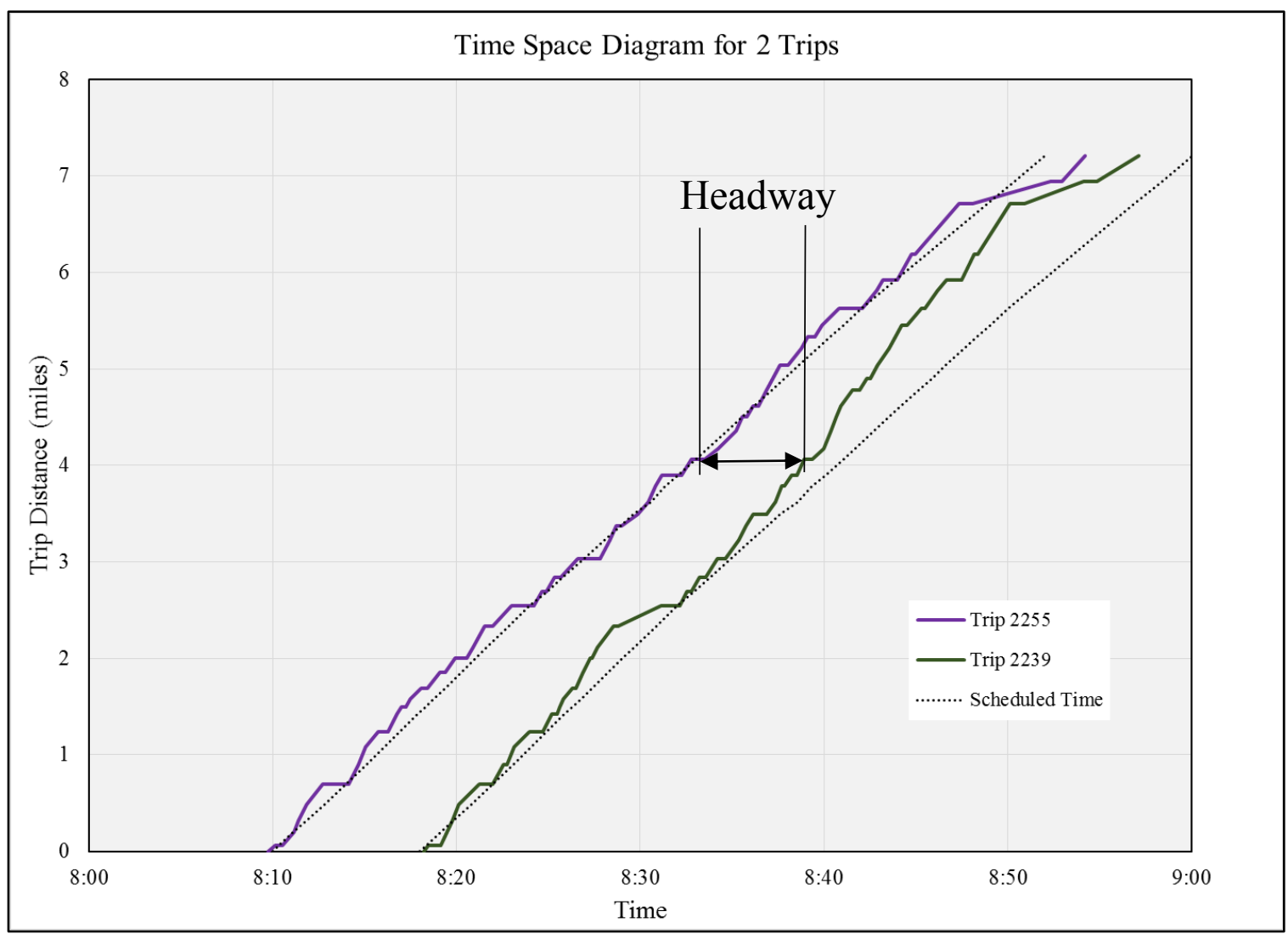

Figure 18: Headway Illustration with 2 Trips

Once the bus deviates from the schedule, the headway decreases between the buses ahead or behind it. The scheduled headways were also calculated to compare the on-time performance. Plots were created to illustrate the differences in headways during different peak periods. As expected, the AM peak period has the smallest headways (lower 
headways correspond with higher frequency of buses). The Late period, after 8 PM, had the largest headways because of the infrequent service. This Late period also has the most on-time performance because of less traffic congestion and passenger movement. The traffic congestion and passenger movement during the morning on the inbound trips is the most impacted; therefore, the AM peak period was analyzed.

In order to set up all of the headways, some points had to be removed. Unscheduled stops had to be adjusted to work with the rest of the data. The majority of unscheduled stops are within 30 seconds of the initial stop time and about 300 feet after the stop location. Approximately $10 \%$ of trips per day had an unscheduled stop along the route. Roughly half of the unscheduled stops may be from GPS miscalculation near the stop or the need for the bus to stop at a traffic signal at the stop location. These are valid assumptions because these points did not have any dwell time or passenger movement. The unscheduled stops without dwell time were removed, or the initial stops were removed if both recordings had dwell time.

Various plots were created to illustrate the actual, scheduled and difference headways for each peak period. The actual headways followed the same flow as the scheduled headways. However, once the difference between the two were plotted, it was evident that there was variation. The difference plot also represents when the buses have a longer or shorter headway. As described in the literature review, bus bunching occurs when one of the buses falls behind and the former gets ahead of schedule, or vice versa. 


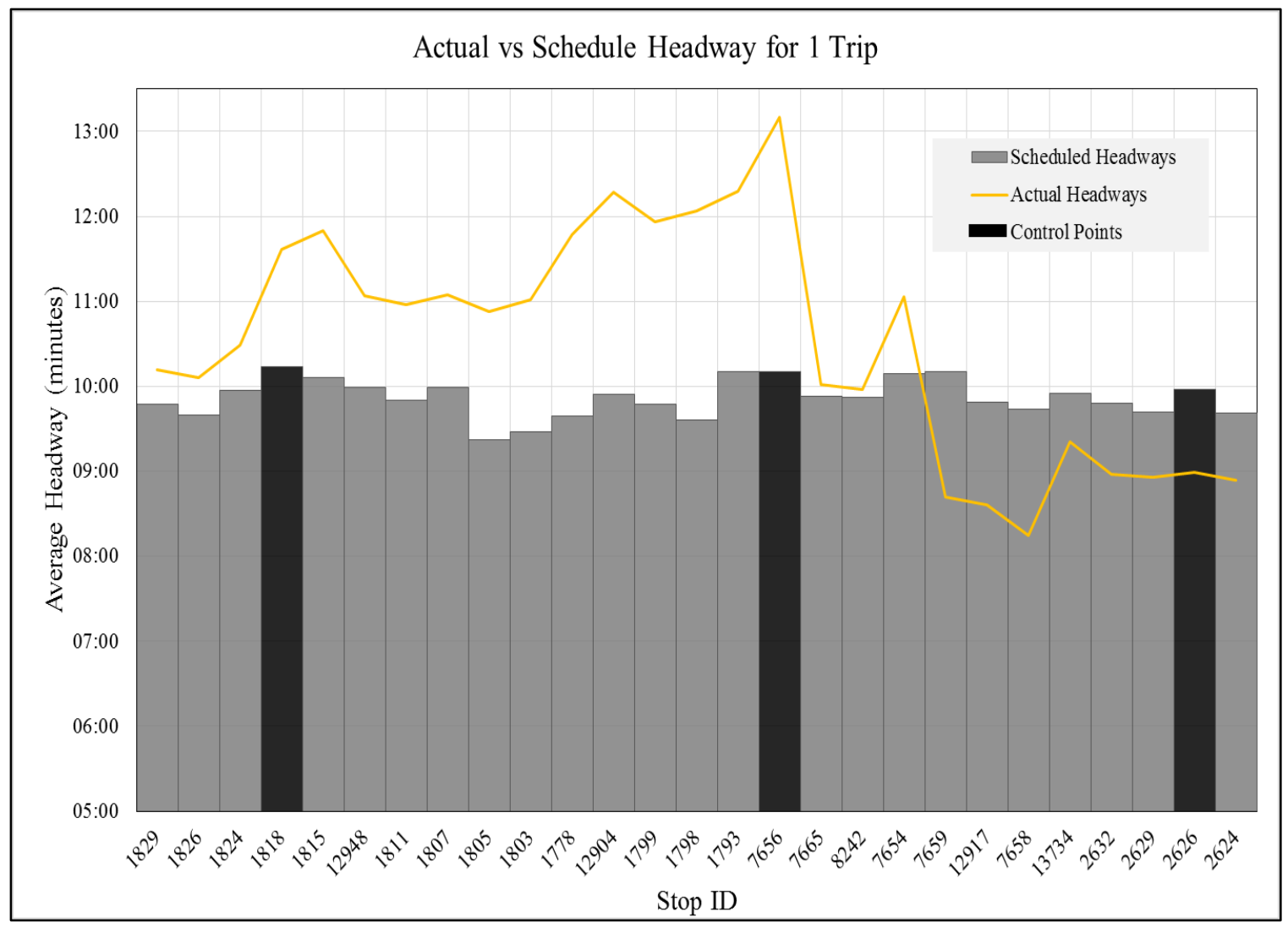

Figure 19: Actual vs Scheduled Headways for 1 Trip

Figure 19 illustrates the actual and scheduled headways between two trips in the AM peak period. The use of connected lines shows an approximate/average change in headway between stops. The scheduled headways are only listed for a few bus stops, the major bus stops, and the rest of the times are interpolated which explains the inconsistency in gray bars. The black bars represent the control points; these are the time points which allow for slack time so buses can get back on schedule. As shown in this plot, the average headway for the AM peak period becomes much higher than the scheduled headway. At the time point in the middle of the route, the actual headway decreases substantially until it matches the scheduled headway. This allowed the late bus to get back on track. The headways shown between stops are simply interpolated from the stop level data. Headway for 5 second data follows a much more complex procedure. 
This will be further explained in the Headway Variation in Chapter 2.3.4.

\subsubsection{Segment Analysis}

There are two segments which are analyzed in addition to the route as a whole. One of the segments is between two major control points and is used to determine the impacts of several independent variables on transit travel time, described in the next section, 2.2.5. As shown in Figure 20, the second segment analysis is performed on Hawthorne Blvd between 47th and 30th street which approximately 1 mile long, including 8 total stops in the inbound direction. BDS data for October 7-9, 2014, was used to estimate the average speed through the segment.

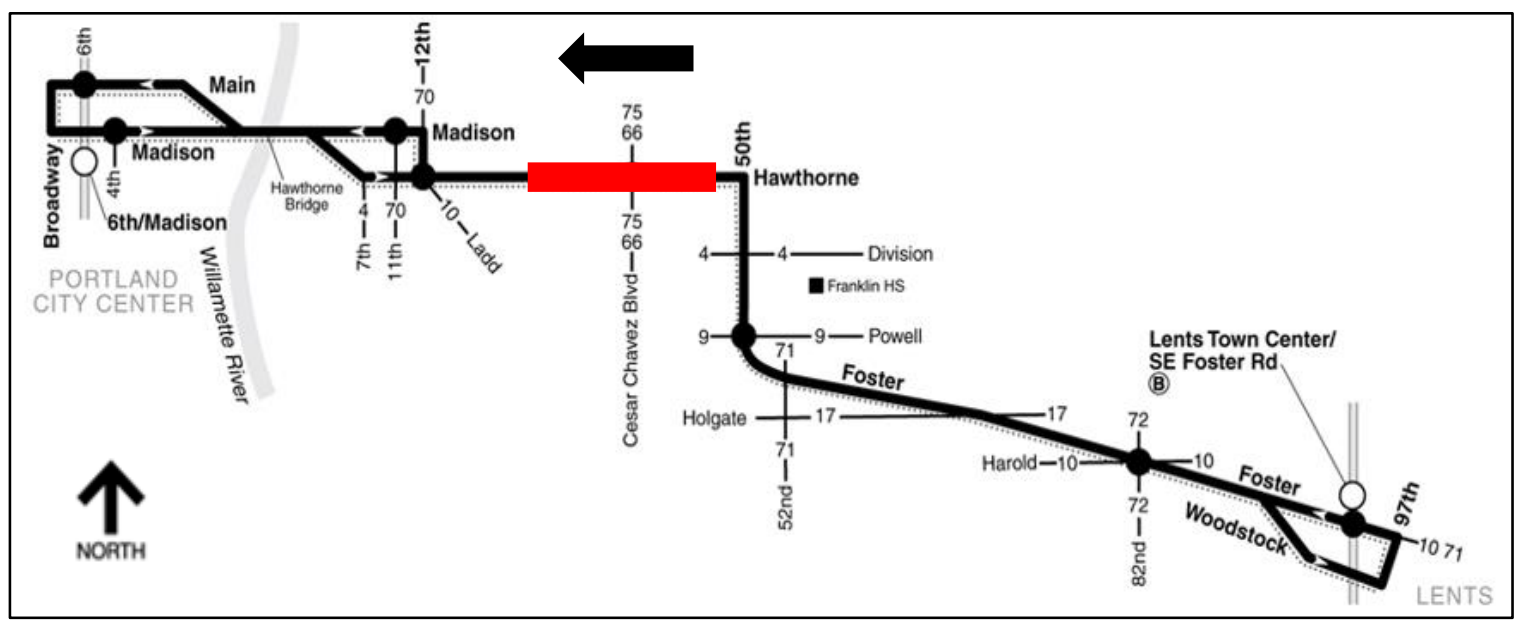

Figure 20: Inter-Stop Segment on Hawthorne Blvd

The segment was chosen based on dwell time, schedule adherence and passenger loading. This portion of the trip has the highest passenger activity as seen by the dwell plots and time-space diagram. This segment also includes the busiest stop which is at a major intersection Cesar Chavez Blvd \& Hawthorne Blvd. The Stop Analysis will be performed at this stop (2619) and it will involve headway analysis and dwell time.

This segment was chosen based on the high dwell time and variation from the schedule time. The first step was to calculate the distance between the stops and the inter- 
stop time for each trip. Next, any errors or trips which have missing information were removed. A box plot was created from the inter-stop times to analyze the variability of travel time between stops. Alternatively, the variability of dwell time is discussed in the next section.

\subsubsection{Travel Time Determinants Preparation}

Transit trip time depends on several factors which can cause early and late buses. Trip times can vary due to large or small passenger movements, traffic congestion, time of day, signal delay, schedule variation or random occurrences along the route. In order to solely focus on the input variables considered, a specific segment was strategically chosen between two critical time points. Transit routes typically include "time points" which are at certain bus stops to allow buses to wait if early, or leave promptly if late. The segment analyzed avoids the holding locations at SE 37th Avenue, after the Cesar Chavez Boulevard intersection, and SE 14th Avenue, before turning onto 12th Avenue. This particular 1.2 mile segment was selected because it provides a mixture of traffic signals, pedestrian crosswalks and travel along the busy Hawthorne Boulevard as shown in Figure 21.

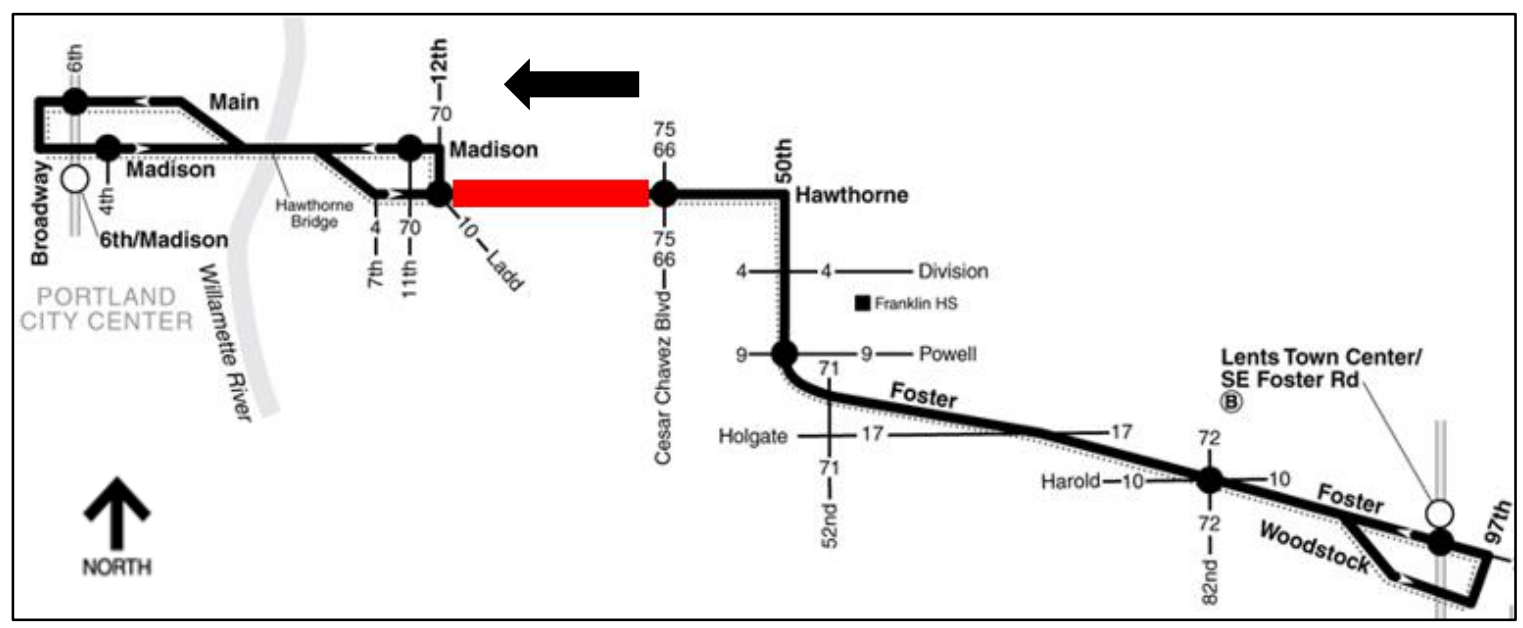

Figure 21: Segment between Critical Time Points on Hawthorne Blvd 
This segment allowed the analysis to capture the real effects of each variable on transit travel time. The data analysis goal is to define the proposed input variables for segment travel time based on readily available data for two work weeks: Tuesday, Wednesday and Thursday for October 7-9 and 14-16, 2014. Originally, only one full week was used for analysis, however, the work week captures more normal conditions compared to different behavior on Mondays and Fridays. Additionally, using work week reduced the number of observations, therefore, two work weeks of BDS data were prepared into a .csv file to estimate regression trees using SAS, statistical analysis software. SAS is a software suite developed by SAS Institute for advanced analytics, multivariate analyses, business intelligence, data management, and predictive analytics. The use of decision trees, developed with SAS, is just one of many tools to determine which factors influence travel time (Park et al., 2015).

Regression tree analysis is a statistical technique that attempts to predict values for a dependent variable based on various independent predictor variables. A tree represents segmentation of the data, created by applying a series of simple if-then rules, data partition as shown in Figure 22.

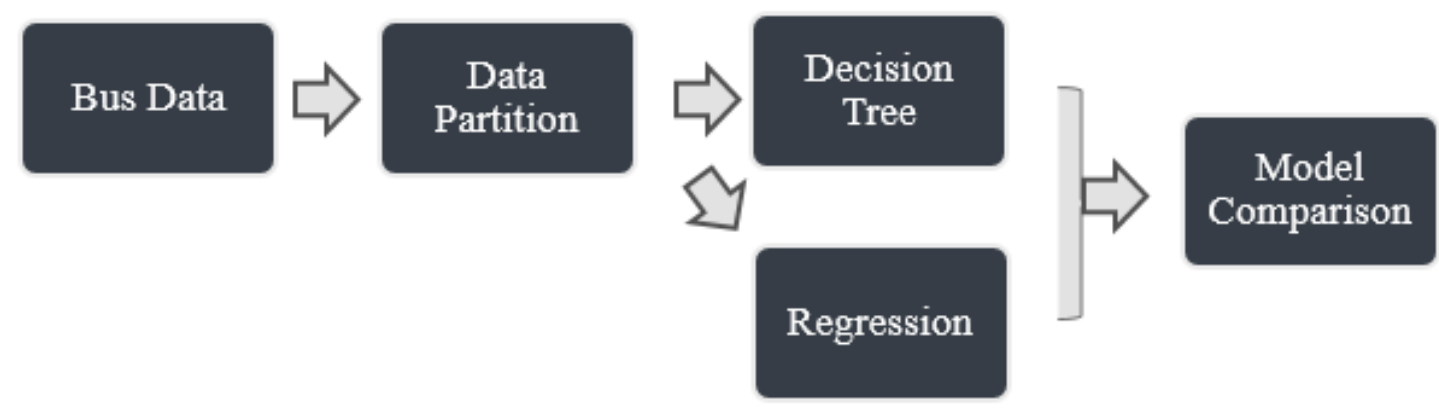

Figure 22: Methodology for Decision Tree Models

Each rule assigns an observation to a group based on the value of one input. One 
rule is applied after another, resulting in a hierarchy of groups within groups. The hierarchy is called a tree, and each group is called a node. The final or terminal nodes are called leaves. For each leaf, the average travel time of all observations in that leaf is the predicted value. Decision trees provide simple interpretable rules for predicting transit travel time based on various characteristics. This methodology does not require any assumptions on underlying distribution of the data and independence of all input variables (Shaaban \& Pande, 2015). Once the travel time determinants are known, it is possible to move forward to build the trip time model.

\subsubsection{Newell Trip Time Model Data Preparation}

Only BDS data is required to replicate the Newell Trip Time Model (Bertini \& ElGeneidy, 2004). The stop level data had to be split into dwell time (serving passengers at the bus stop) and inter-stop time (time spent between stops). Before creating the model, filters were to remove trips with lifts or any other errors such as missing estimated load, missing boardings, missing alightings or missing speeds. Separate data for only boardings and only alightings were filtered out in order to recreate the dwell time model portion of the trip time model. The arrival times of the bus were subtracted by the leave times at the previous stops for the inter-stop portion. The Newell trip time model is further explained and developed in Chapter 4.

\subsection{Second Resolution (5SR) Data}

In addition to the BDS data, the next dataset obtained was the new, 5 second resolution data, as shown in Figure 23. This dataset is complimentary to the original BDS, in that it does not include all of the vehicle identifiers, it solely contains a timestamp and GPS location of the bus up to every 5 seconds. However, when the bus 
travels below $5 \mathrm{mph}$, the data is not collected until the bus travels above $5 \mathrm{mph}$.

In order to work with this data, the time was converted into hours, minutes, and seconds. The gap time, time between each recording, was also calculated by subtracting consecutive data points recorded. Although this is considered 5 second resolution data, the cells in red in Table 3 represent either greater or less than 5 seconds. Also, the $x$ - and $y$-coordinates of the GPS locations had to be converted into trip distance using the same procedure as with the BDS data as described in section 2.2.1). BDS data output one trip in 45 rows, this dataset outputs approximately 300 rows for one trip (over 1.5 million rows of data per month).

\begin{tabular}{|c|c|c|c|c|c|c|c|}
\hline $\begin{array}{l}\frac{9}{\mathrm{U}} \\
\frac{\mathrm{U}}{\mathrm{I}} \\
\mathrm{g}\end{array}$ & 㻤 & 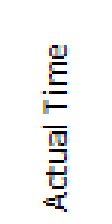 & 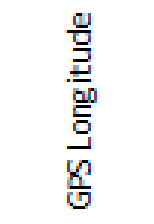 & 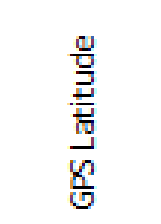 & 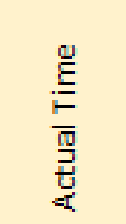 & $\frac{\stackrel{g}{g}}{\stackrel{g}{E}}$ & 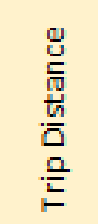 \\
\hline 2005 & Oct 6,2014 & 28957 & -122.566 & 45.47918 & $8: 02: 37$ & 0 & 0 \\
\hline 2005 & Oct 6,2014 & 28962 & -122.567 & 45.47924 & $8: 02: 42$ & 5 & 0.011 \\
\hline 2005 & Oct 6,2014 & 28965 & -122.567 & 45.47925 & $8: 02: 45$ & 3 & 0.012 \\
\hline 2005 & Oct 6,2014 & 28967 & -122.567 & 45.47925 & 8:02:47 & 2 & 0.013 \\
\hline 2005 & Oct 6,2014 & 28972 & -122.567 & 45.47933 & 8:02:52 & 5 & 0.026 \\
\hline 2005 & Oct 6,2014 & 28977 & -122.567 & 45.47947 & 8:02:57 & 5 & 0.048 \\
\hline 2005 & Oct 6,2014 & 28982 & -122.567 & 45.47956 & 8:03:02 & 5 & 0.061 \\
\hline 2005 & Oct 6,2014 & 29007 & -122.568 & 45.47959 & 8:03:27 & 25 & 0.067 \\
\hline 2005 & Oct 6,2014 & 29012 & -122.568 & 45.47963 & 8:03:32 & 5 & 0.079 \\
\hline 2005 & Oct 6,2014 & 29017 & -122.568 & 45.47968 & 8:03:37 & 5 & 0.088 \\
\hline 2005 & Oct 6,2014 & 29022 & -122.568 & 45.47969 & 8:03:42 & 5 & 0.090 \\
\hline 2005 & Oct 6,2014 & 29027 & -122.568 & 45.47977 & 8:03:47 & 5 & 0.103 \\
\hline 2005 & Oct 6,2014 & 29032 & -122.569 & 45.47993 & 8:03:52 & 5 & 0.130 \\
\hline 2005 & Oct 6,2014 & 29037 & -122.569 & 45.48013 & 8:03:57 & 5 & 0.165 \\
\hline 2005 & Oct 6,2014 & 29047 & -122.571 & 45.48057 & 8:04:07 & 10 & 0.240 \\
\hline 2005 & Oct 6,2014 & 29052 & -122.572 & 45.48078 & 8:04:12 & 5 & 0.277 \\
\hline 2005 & Oct 6,2014 & 29057 & -122.572 & 45.48095 & $8: 04: 17$ & 5 & 0.309 \\
\hline 2005 & Oct 6,2014 & 29077 & -122.572 & 45.48105 & 8:04:37 & 20 & 0.325 \\
\hline 2005 & Oct 6,2014 & 29082 & -122.573 & 45.48116 & $8: 04: 42$ & 5 & 0.346 \\
\hline 2005 & Oct 6,2014 & 29087 & -122.574 & 45.48134 & 8:04:47 & 5 & 0.380 \\
\hline 2005 & Oct 6,2014 & 29092 & -122.574 & 45.48152 & 8:04:52 & 5 & 0.419 \\
\hline 2005 & Oct 6,2014 & 29097 & -122.575 & 45.4817 & $8: 04: 57$ & 5 & 0.458 \\
\hline
\end{tabular}

Figure 23: Example of 5SR Data 


\subsubsection{Second Data Preparation}

Using the BDS data as a guide, the 5 second resolution data was extracted by comparing the times recorded on both datasets. The 5 second resolution data does not start and stop collecting data at the beginning and end of a trip; the BDS gives the start and stop times for each bus and allows the 5 second resolution data to be aligned for a complete trip. Although this was prepared manually in order to ensure minimal errors, a macro can be set up in Excel to match the start and end times of the stop level data to clean up and align with the 5 second data. Most rows of data which contained zero for the distance traveled over 5 seconds were removed. The majority of runs were consistent with 7.2 miles traveled. All of the trips for October 7-9 and 14-16, 2014, in the 5 SR data averaged 7.20 miles and 39 minutes 43 seconds for the total trip time (total $n=382$ ). In order to determine whether the 5 second data represented the same trip as the stop level data, both datasets were plotted on the same graph as shown in Figure 24. 


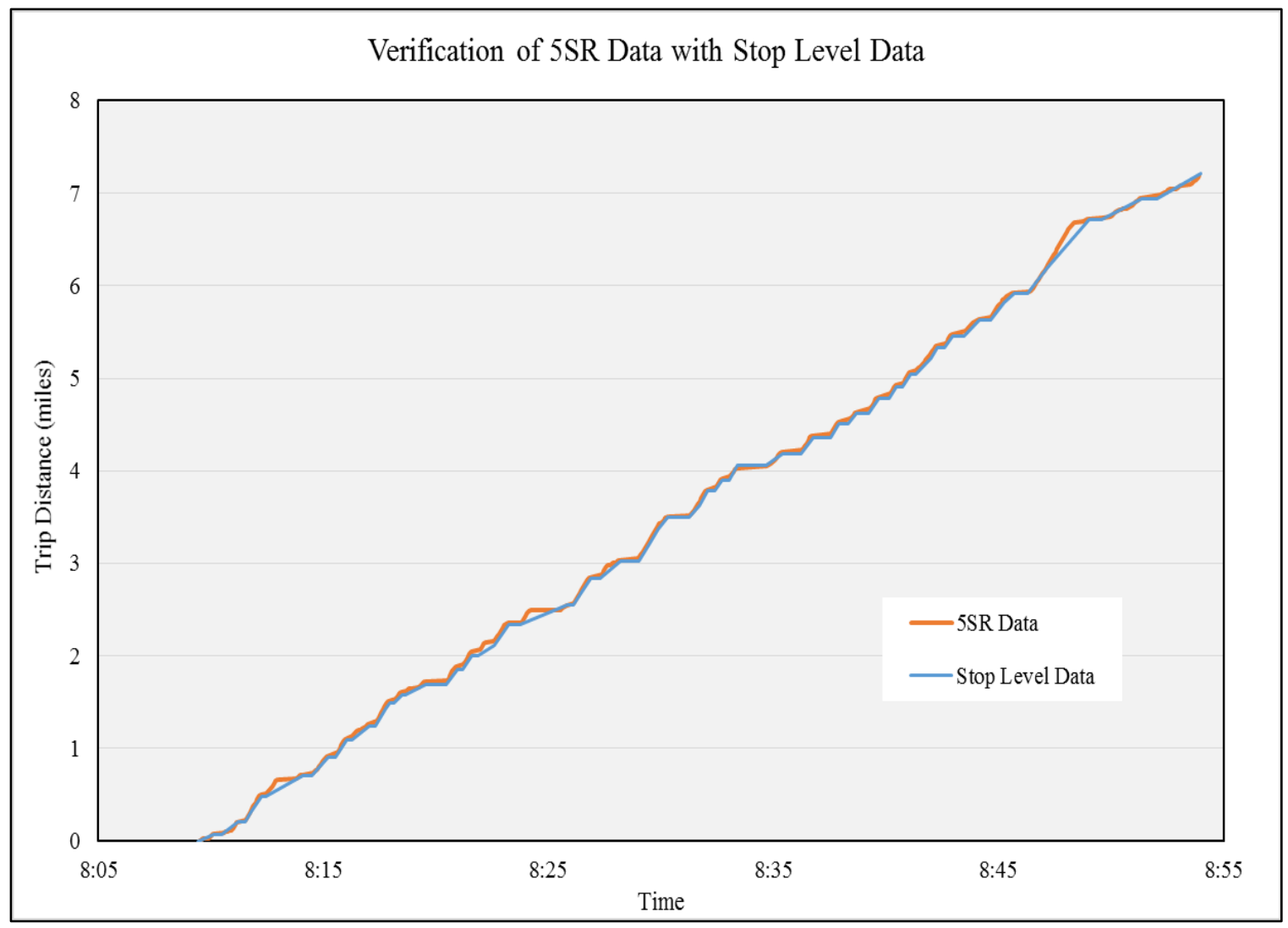

Figure 24: Verification of 5SR Data with Stop Level Data

This figure illustrates the higher resolution of the 5 second data by showing its fluctuations above and below the stop level data. As mentioned in the previous section, the stop level data collects data at each stop and assumes an average between the two points. This data provides further insight as to what occurs between the stops; however, it does not display this information using the raw 5 second resolution dataset as shown in Figure 25 . 

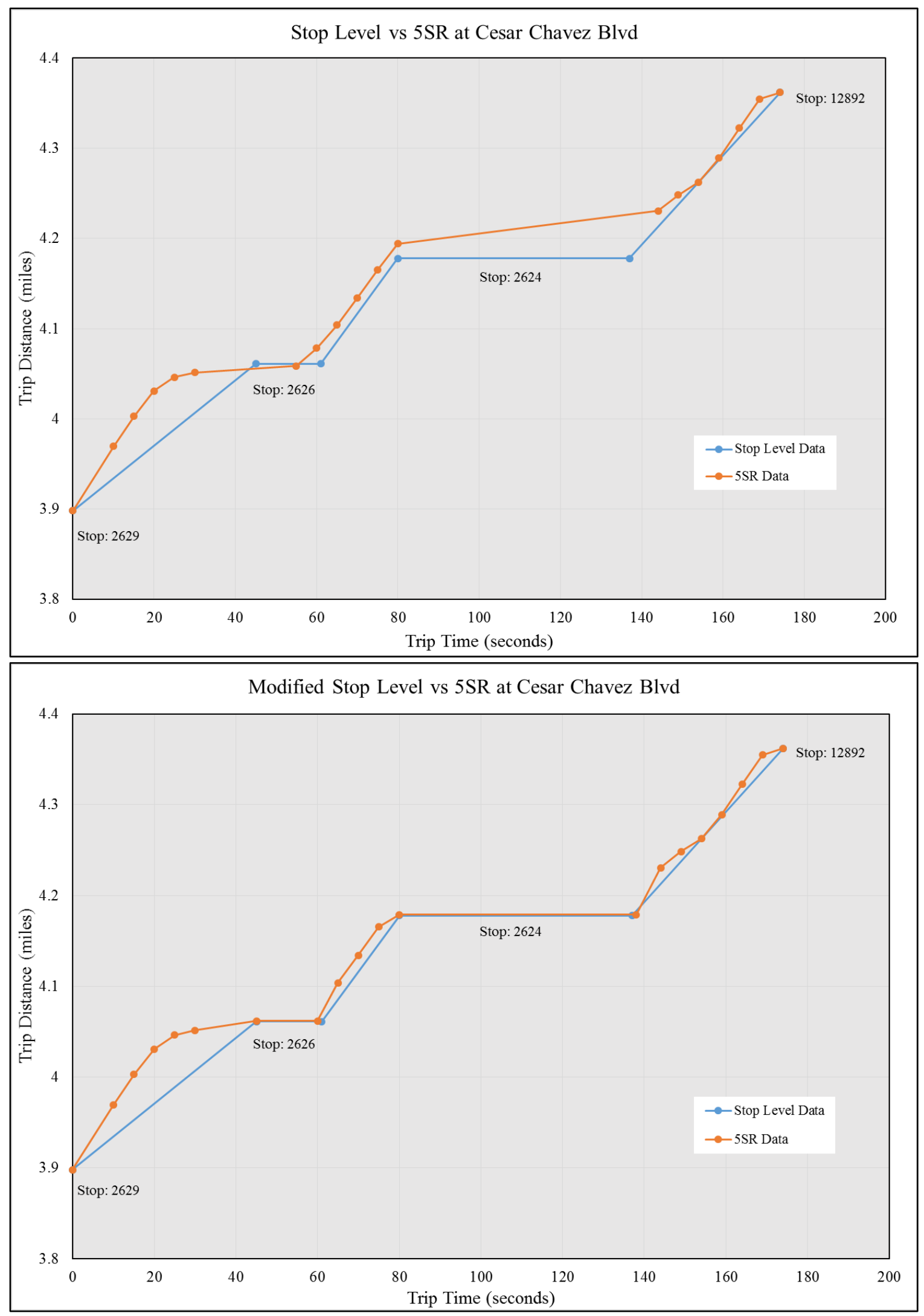

Figure 25: Raw Stop Level Data vs 5SR Data (Top) Modified 5SR Data (Bottom) 
The raw 5 second resolution data does not depict realistic conditions as shown in the top of Figure 25. Once each trip is verified from start to end using the stop level data, it must be aligned in order to shift the original 5 second resolution points to the actual stop locations. As mentioned in the previous sections, stop level data is exceptional for time and location at the stop. Therefore, it is necessary to match the 5SR data points with the BDS data time and location at the bus stop locations to use it effectively. After the stop locations are aligned, the 5SR data represents realistic conditions as shown in the bottom of Figure 25. Between the two stops, the bus appears to travel faster than the average speed, as depicted by the stop-level data, and then the bus slows down to the bus stop at 2626, which portrays realistic conditions.

\subsubsection{Travel Speed Analysis}

Speed is an integral component of total trip time (TCRP 88, 2003). The average speed between each stop and the overall average speed can impact the total trip time. Using the BDS data, the average speed is calculated based on the arrival and departure times at the stop locations. Although the BDS data includes an approximate maximum speed traversed by the bus between stops, this may only be achieved for an instant before slowing down for traffic conditions, a signal or a crosswalk.

The AM trip distances were extracted from the data in order to analyze the morning peak periods of $6 \mathrm{AM}$ to $10 \mathrm{PM}$. The inbound route typically has the highest usage, and lowest reliability because of the congestion from commuters. Trips which included errors were removed, such as trips with reported 13 lifts used and trips with missing passenger movement information. The morning period will be analyzed using BDS data and compared to the 5-second resolution data. The speed plots were created to 
compare the average and maximum speeds for an entire day and the AM peak period. The maximum and average speed for an entire day were 46.1 and $19.4 \mathrm{mph}$, respectively. The maximum and average speed for the AM peak period were 46.1 and $19.0 \mathrm{mph}$, respectively. Figure 26 illustrates the breakdown of average speeds for the AM peak period for 3 days: October 7-9, 2014.

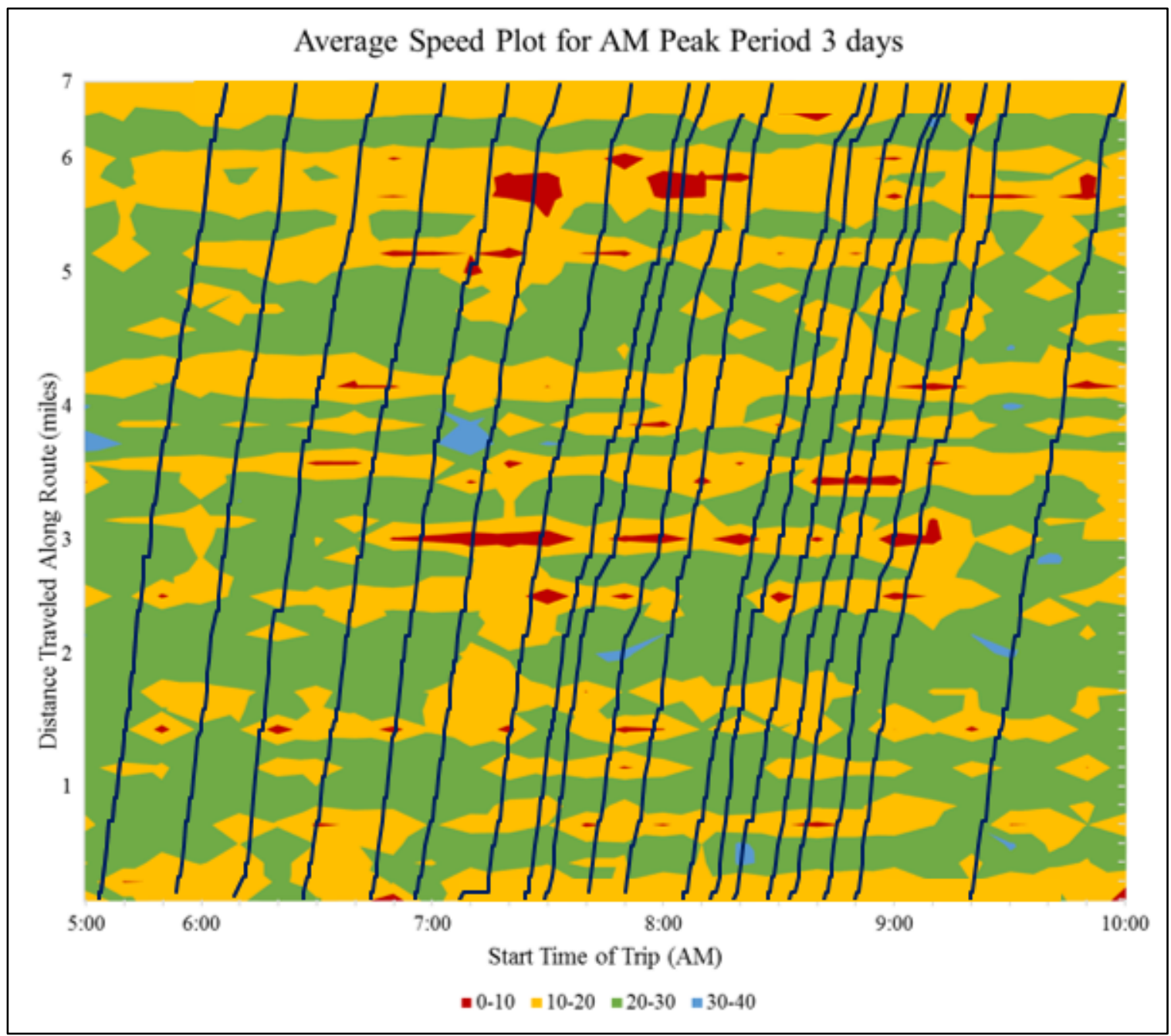

Figure 26: Average Speed Plot for AM Peak Period

The heat map represents speeds using stop level data overlaid with bus trajectories. The speeds between 0-10 mph are in red, 10-20 mph are in yellow, 20-30 mph are in green and 30+ mph are in blue. The speeds around mile 3, bus stop 7654 at SE 
50th and SE Division Street, are typically below $10 \mathrm{mph}$. These speeds are low from 6:55 AM to 7:40 AM most likely due to the traffic conditions during the morning commute. The average speeds were used for three days of data, but these speeds were simply averages between stops.

In order to understand the bus behavior between stops, the 5 second data is required to analyze the speeds. Figure 27 compares average speeds for the work week of October 7-9, 2014 using BDS and 5SR data.

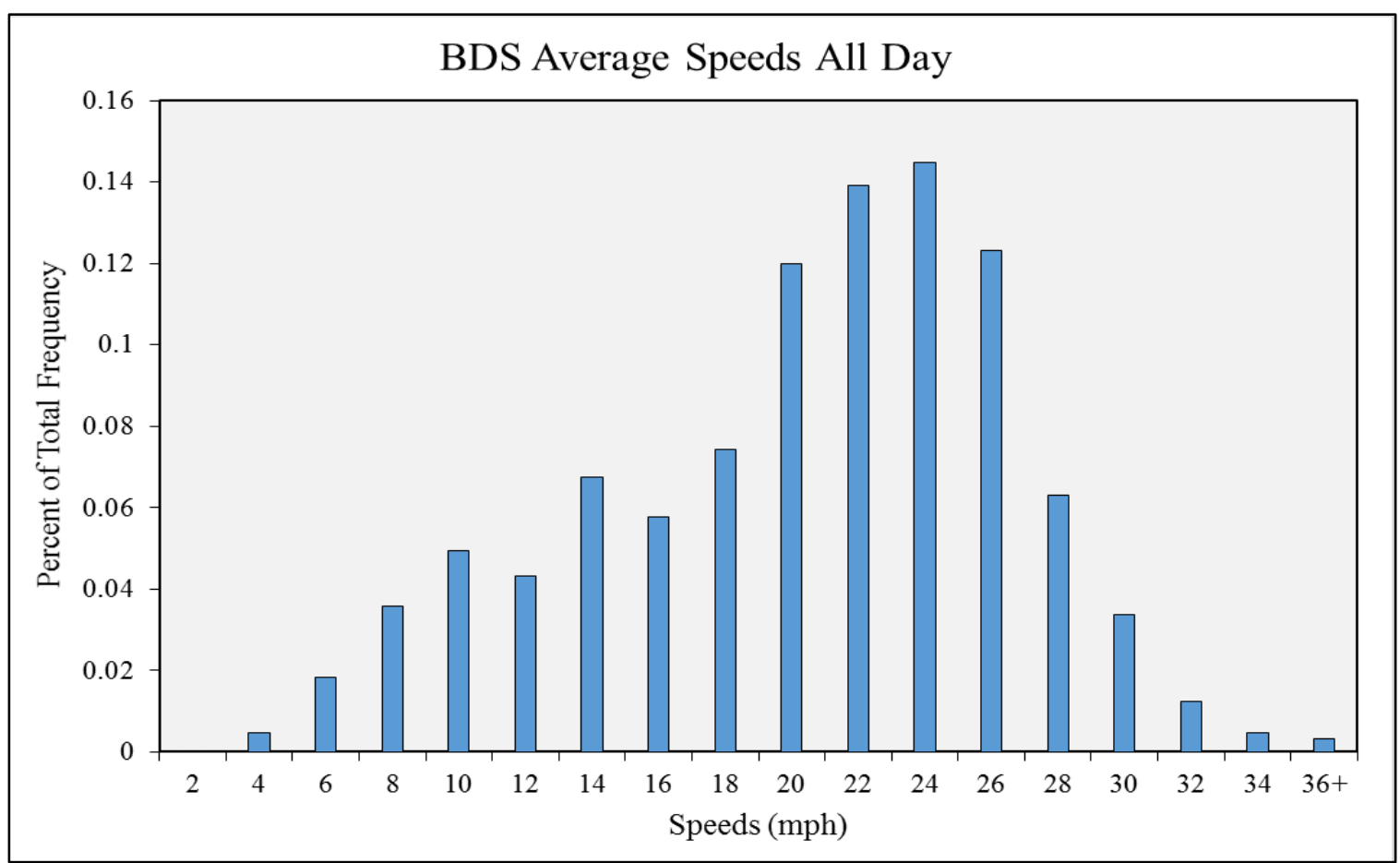

Figure 27: Average Speed Histogram using BDS Data 


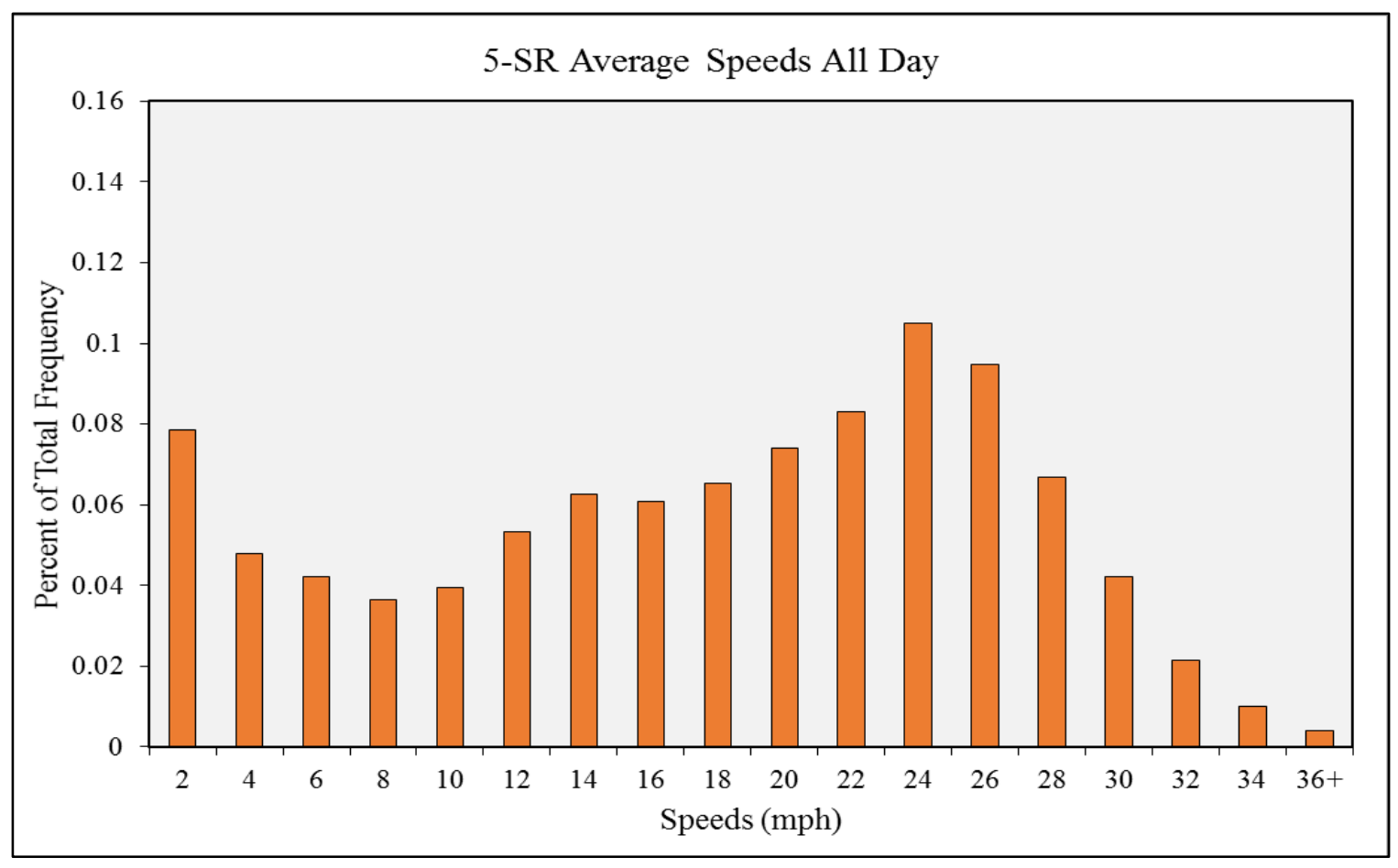

Figure 28: Average Speed Histogram using 5SR Data

The average speeds using BDS data displayed higher average speeds compared to the 5second resolution data. The $5 \mathrm{SR}$ speeds are averaged over 5 seconds compared to the BDS data which averaged the speeds over 30-90 seconds. The average distance between each stop is 0.16 miles compared to 0.025 miles difference between 5 -second resolution data points. There is an opportunity to analyze actual speeds between the segments instead of relying on the average speed between stops from the stop level data. Increased detail of speeds between stops allows transit authorities to discover trends and travel patterns throughout the route. Speed variation is further described in Chapter 4 for transit trip time models.

The following steps were performed in order to estimate the average speed over three days, October 7-9, 2014. The speed was analyzed between stops, in between the segment: 47th and 30th Avenue on Hawthorne Boulevard as shown previously, in Figure 20. The analysis is during the AM peak period, from 8 AM to 9 AM. One trip was chosen 
to average and compare over three days. The 5-second resolution data was matched up to the stop level data. The stop level data on October 7 , vehicle 2270 started at 8:17:35, on October 8, vehicle 2305 started at 8:17:55, and on October 9, vehicle 2527 started at 8:14:10 AM. In conjunction, the 5 second resolution trips started at 8:17:37, 8:18:00 and 8:14:11 AM, respectively. The average gap was calculated over the three days for 5second resolution. Any gap that was over 5 seconds was removed because any gap greater than 5 seconds typically represents when the bus is at a stop (dwell time). Next, the average trip distance was calculated for the three days. The stop level data required using the arrival times and dwell times. Subtracting the subsequent arrival time with the current arrival time includes the time spent at the stop, therefore, the dwell time was also subtracted to end with the gap times between stops. Similarly the gap times and trip distances were averaged over the three days as shown in Figure 29. 


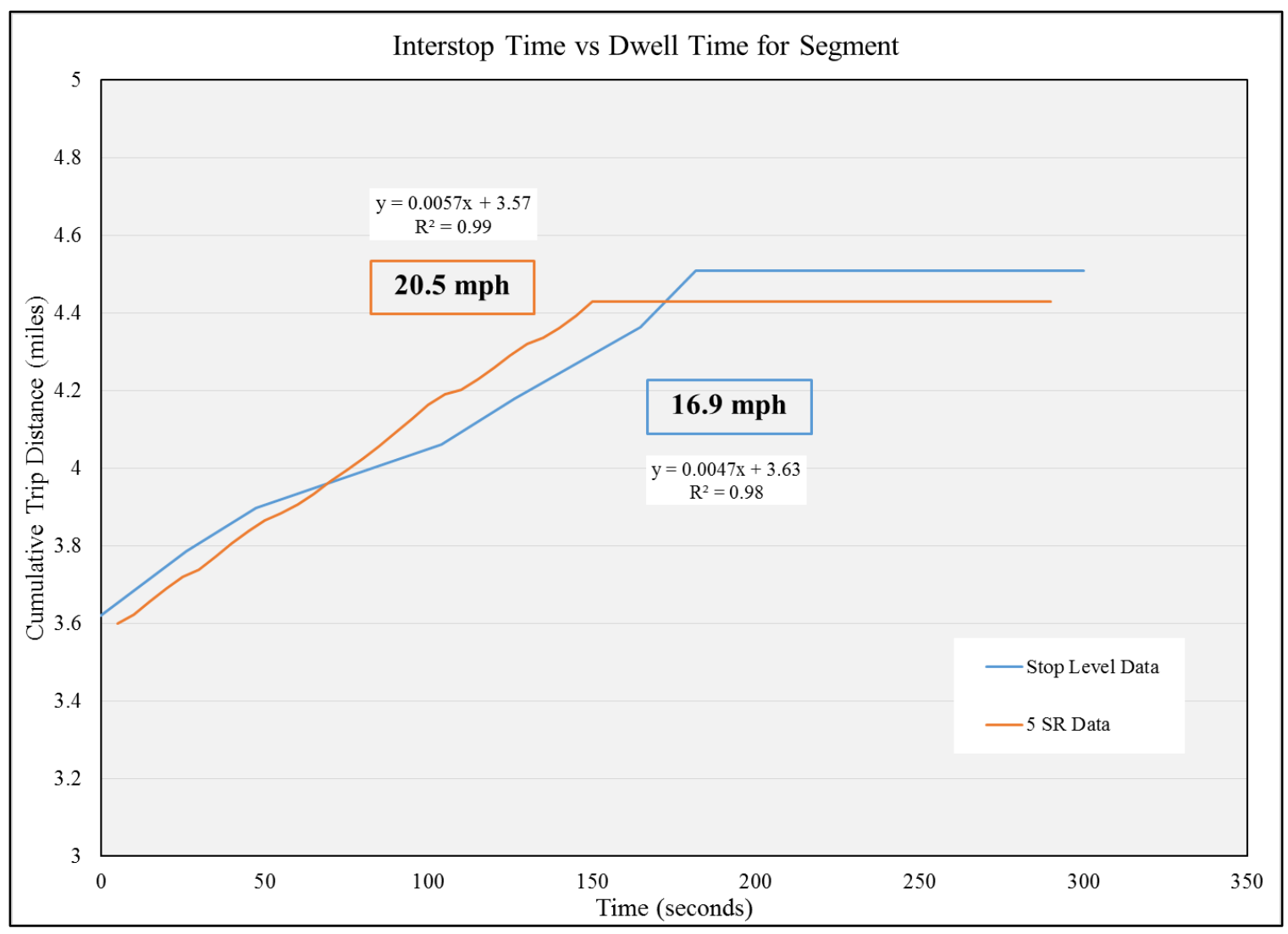

Figure 29: Inter-Stop Time vs Dwell Time for Segment

The inter-stop speed plot represents the gap time between stops vs cumulative trip distance for the stop level data and the 5-second resolution data. The average speed is determined by multiplying the slope of each line by 3600 seconds/hour because the plot is in miles per second. Linear regression was used to determine the equation of each line and the results were $20.5 \mathrm{mph}$ for 5 -second resolution and $16.9 \mathrm{mph}$ for stop level data.

\subsubsection{Acceleration and Deceleration}

Each time a bus stops, it must accelerate and get back into the flow of traffic. Contrarily, the bus must decelerate in order to stop at the bus stop. Stop level data does not provide this information; it simply takes the first stop timestamp and the next stop timestamp to determine the average speed. Figure 29 illustrates the accelerations and decelerations using 5 second resolution data for an entire day, Tuesday October 7, 2014. 
The majority of the accelerations are between -1 to +1 , which is reasonable because the bus does not typically accelerate or brake drastically unless there is an emergency.

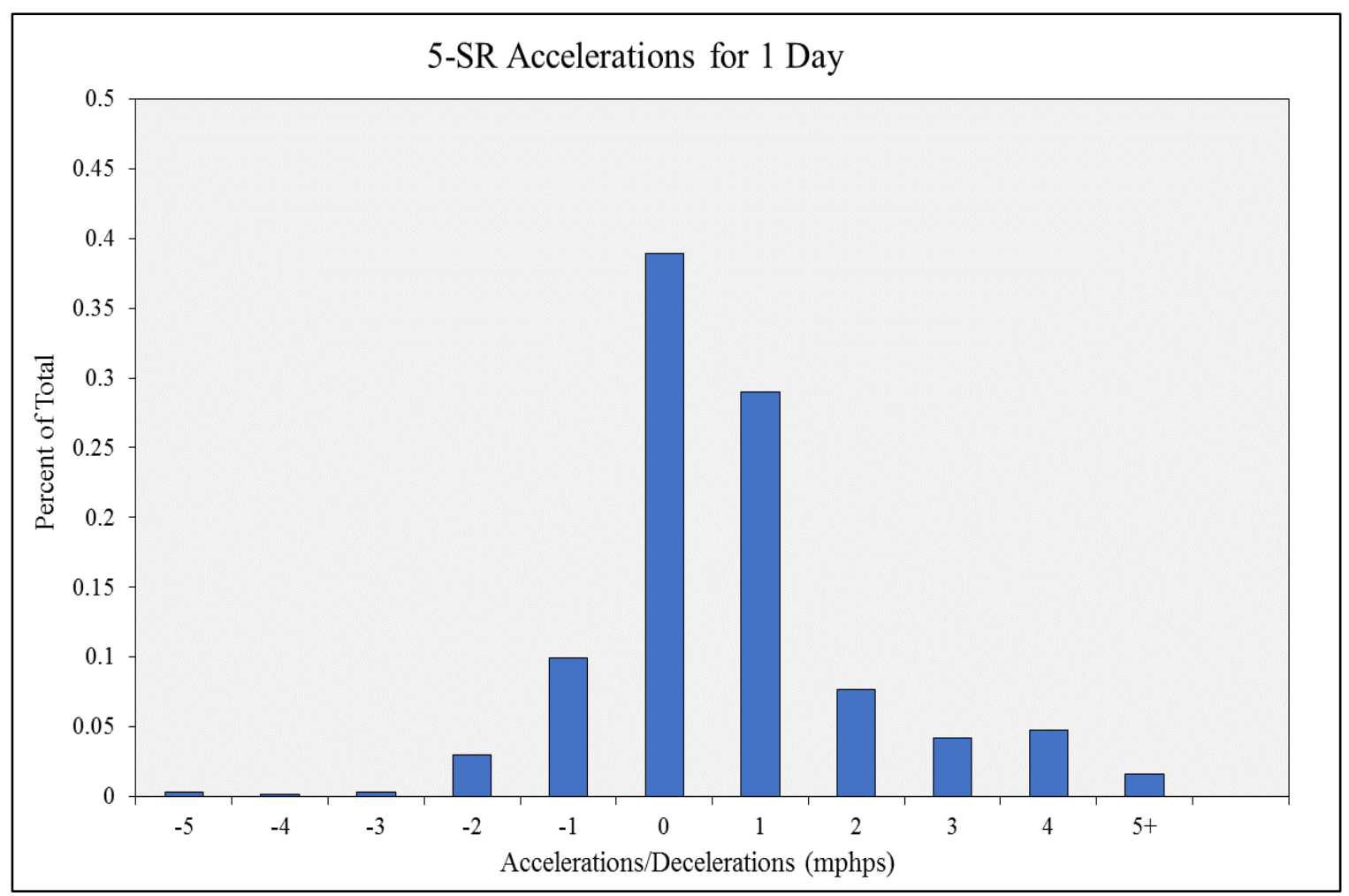

Figure 30: Histogram of Accelerations using 5SR Data

The time for the bus to each cruise speed can be calculated by using the 5SR data after each stop. This is determined from the time the bus starts to leave the stop to once the acceleration between the data points is below 2 mphps, to establish and maintain a steady speed.

\subsubsection{Headway Variation}

Higher resolution data should be able to deliver higher resolution information on headways between buses. Stop level data simply uses the stop information to determine the headways between buses, however, it is unknown how the headways vary between stops. Using the raw 5SR to determine the headways between stops does not match with the stop level data. As mentioned previously, stop level data provides accurate 
information at the stops; therefore, if the 5 second data does not match up to the stop locations, it is erroneous as shown in Figure 31.

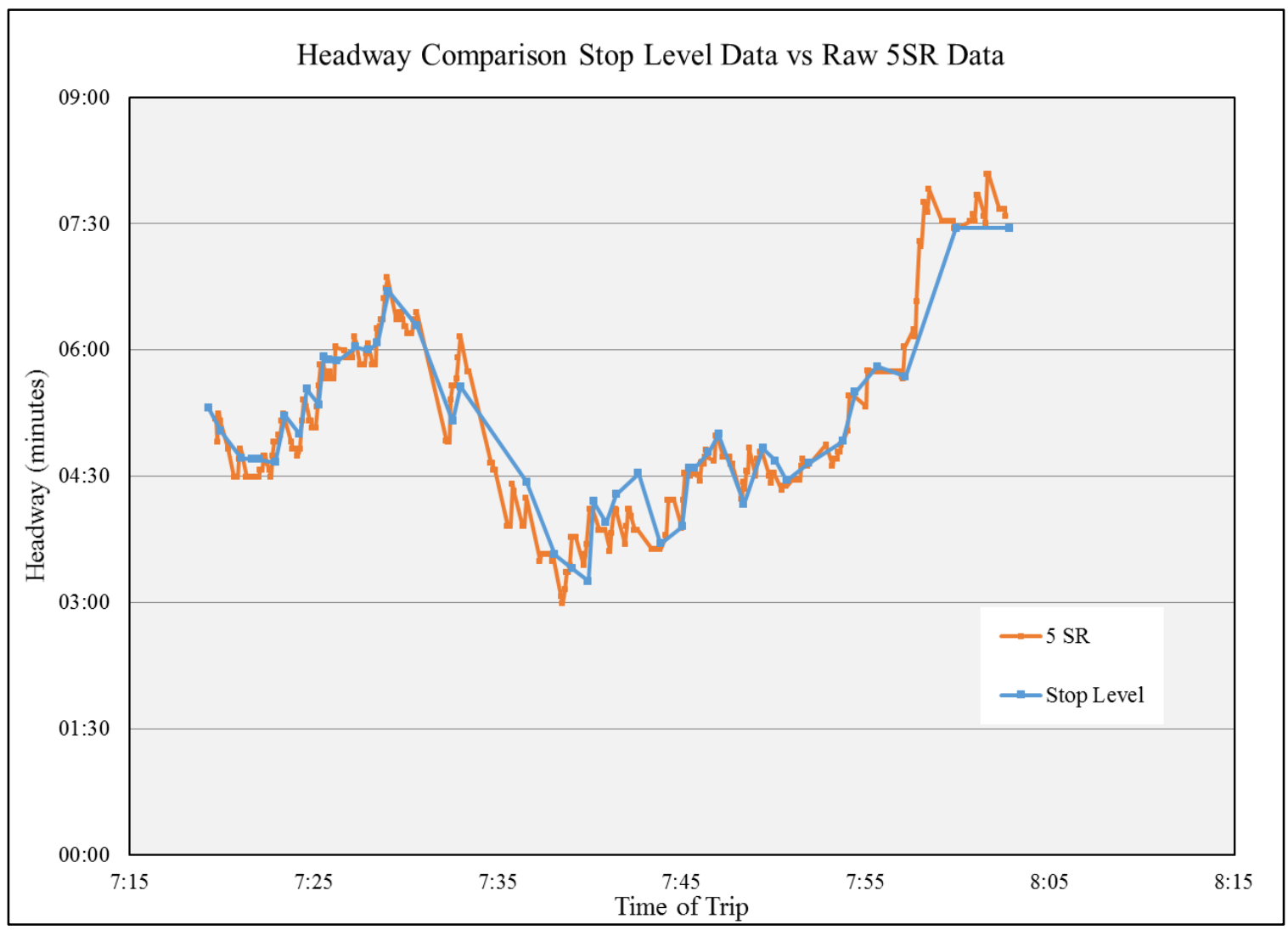

Figure 31: Headway Comparison of Stop Level Data vs Raw 5SR Data

The raw 5 second data is very close to the stop level data, but it appears to miss some stops. The aggregate data had to be modified in order to portray realistic conditions. The 5 second data was split into 1 second increments using a 1 second interpolation method as shown in Appendix E. The 5 second resolution data points were aligned with all stops, and each 5 second interval was split into 1 second and the average was taken between them. Figure 32 uses this 1 second interpolated data to illustrate the higher resolution for headway variation. 

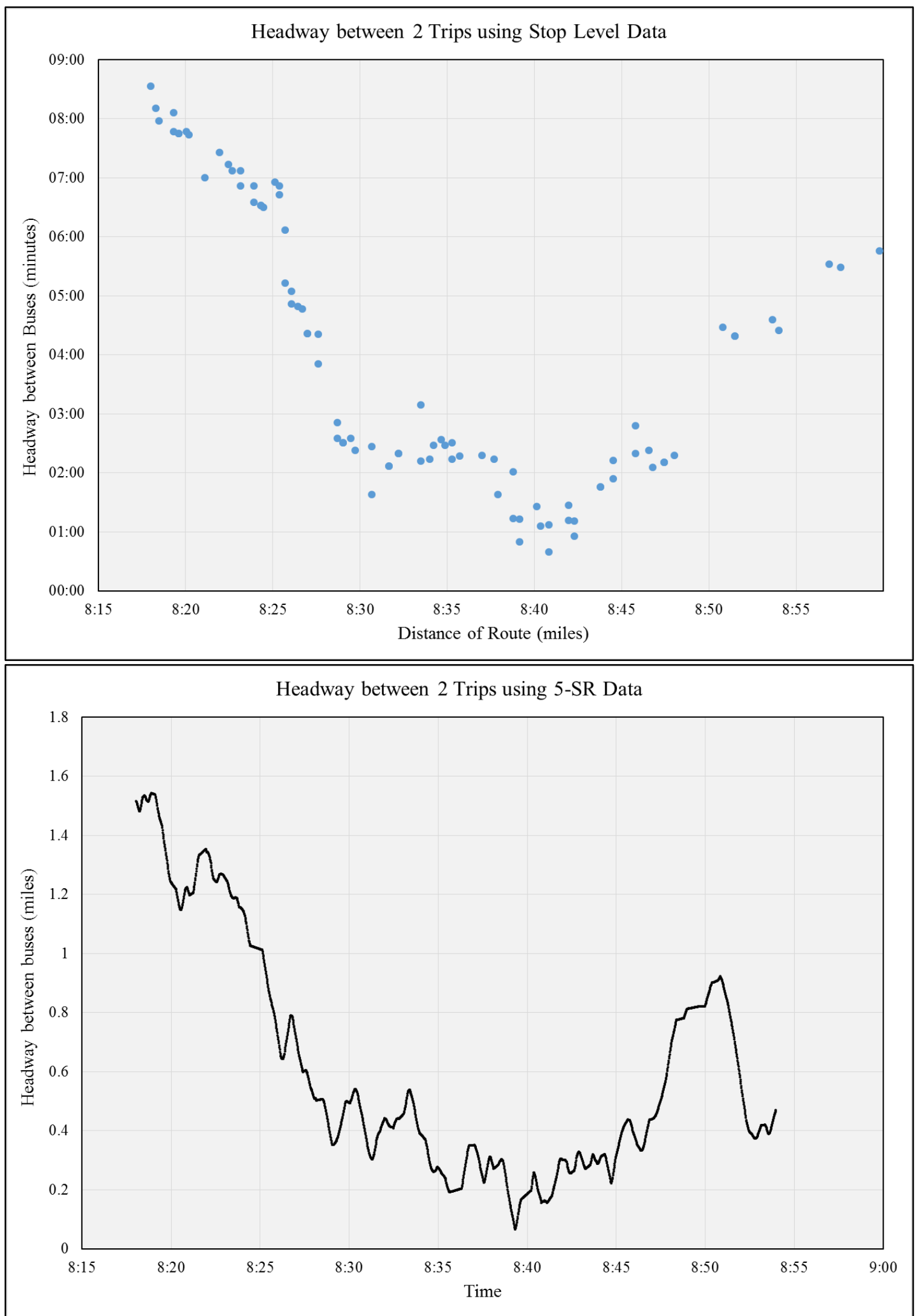

Figure 32: Headway for 2 Trips using Stop Level Data (Top) 5SR Data (Bottom) 
It may appear that the bottom of Figure 32 has connected lines and the top has scattered points, however, both plots have scattered points format. The bottom of Figure 32 uses higher resolution data points to determine the headway at every second of the trip. This allows further insight as to what occurs between stops: the headway drops below 0.1 miles. Similar to speeds between stops, the headway between stops provides greater detail between stops. The next section compares the actual trajectory of the bus to the average trajectory from the stop level data.

\subsubsection{Average vs Actual Trajectory}

5 second resolution allows deeper understanding of what occurs between stops. Stop level data is exceptional for passenger movements and stop information, however, there is ambiguity of what occurs between the stops. Rather than relying on the average speed between stops, the higher resolution data helps identify what happens between stops. Figure 33 illustrates stop level data and 5 second data for one trip segment. This trip segment is for the first 2.5 miles of the trip with seconds as the dependent variable. 


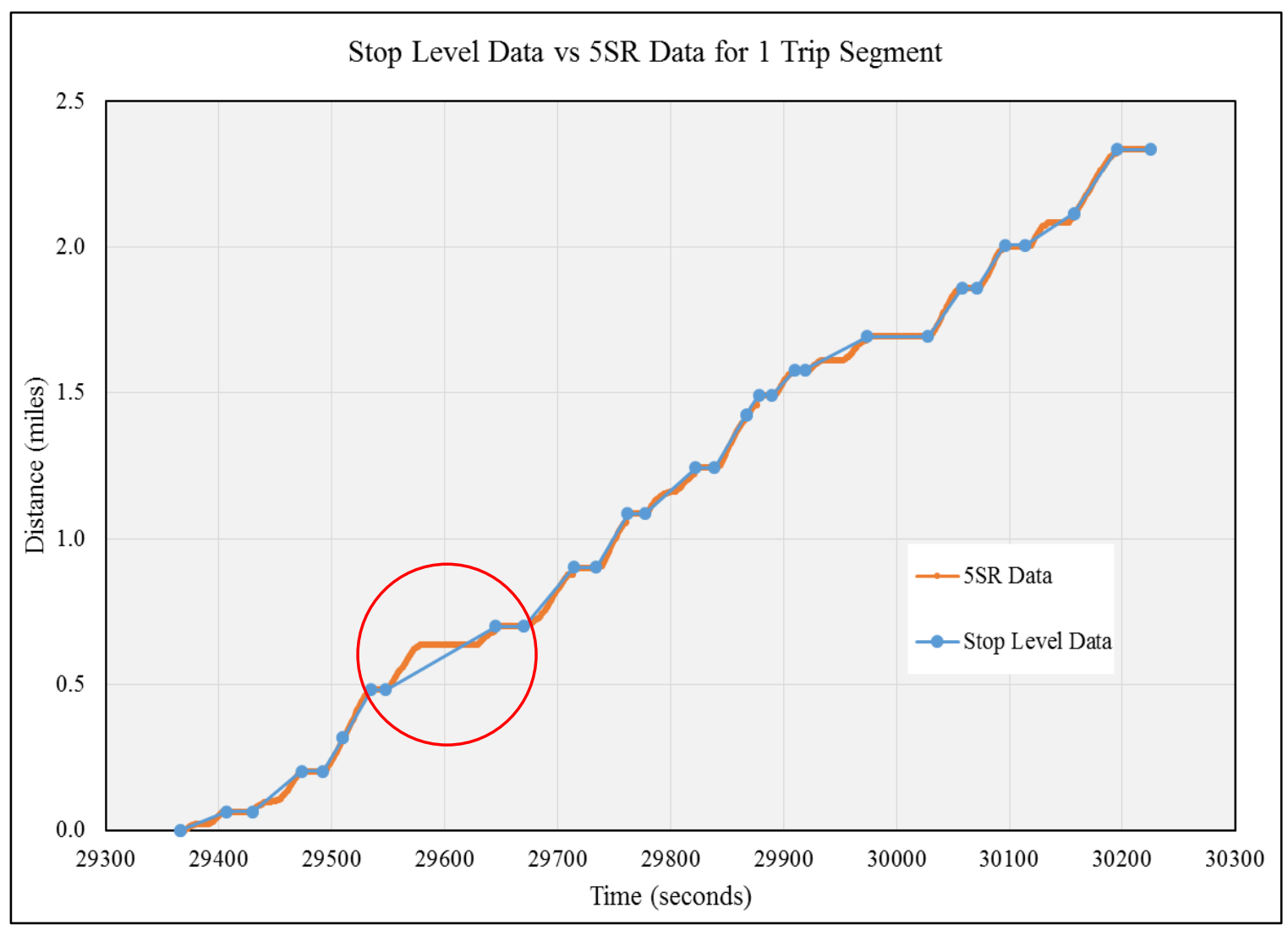

Figure 33: Stop Level Data vs Modified 5SR Data for 1 Trip

The red circle identifies the location of a large deviation from the stop level data and the 5 second data. This segment between stops presumably had signal delay, which would not be apparent simply using the stop level data. Larger variations from the average trajectories arise near signalized intersections as shown in Figure 34. 


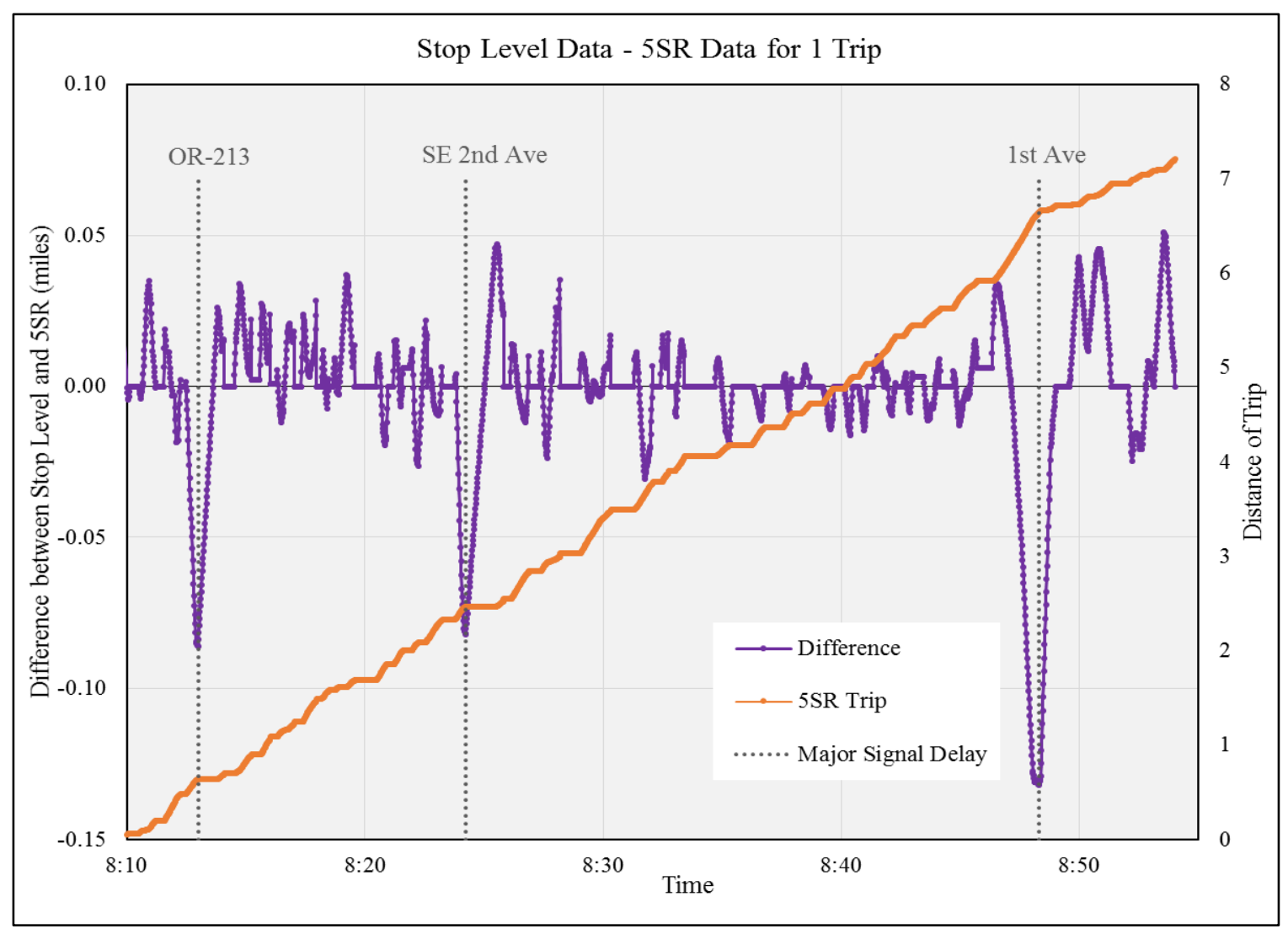

Figure 34: Stop Level Data-Modified 5SR Data for 1 Trip

Figure 34 illustrates the difference between the stop level data and the 5 second resolution data for an entire trip on Tuesday, October 7, 2014. As the bus approaches intersections, the difference increases, especially at signalized intersections. The three major intersection which caused delays for this particular trip were OR213, SE 2nd Ave and 1st Ave. All of the smaller deviations from the zero difference line indicate segments where the bus may have started off quickly and slowed down to the next stop, or vice versa. As the bus reaches a bus stop, the difference becomes zero, which represents the dwell time. The variables that affect the transit travel time are determined in the next chapter. 


\section{FACTORS AFFECTING TRANSIT TRAVEL TIME}

The framework developed here should be of particular interest to transit agencies that are interested in identifying the factors that affect travel time during different times of the day. The data analysis goal is to define the proposed input variables for segment travel time based on readily available data for two work weeks: Tuesday, Wednesday and Thursday for October 7-9 and 14-16, 2014 as described in Chapter 2.2.5. This dataset included 384 total inbound trips with approximately 18,000 boardings and 16,000 alightings over the six day period.

In order to solely focus on the input variables considered, a specific segment was strategically chosen between two critical time points as mentioned in Chapter 2.2.5. As shown previously in Figure 21, this segment allowed the analysis to capture the real effects of each variable on transit travel time.

\subsection{Variable Inputs}

The nine initial variables taken into account included early/late (binary), time period (nominal), start time of trip, schedule deviation, total boardings, total alightings, maximum onboard, total dwell time and average speed, presented in Table 2.

Table 2: Segment Data Summary

\begin{tabular}{|l|c|c|c|c|}
\hline \multicolumn{1}{|c|}{ Variable } & Type & Unit & Mean (seconds) & Standard Deviation \\
\hline \hline Segment Travel Time & Continuous & Seconds & 380.5 & 53.6 \\
\hline Early/Late & Binary & $0-1$ & - & - \\
\hline Time Period & Nominal & $1-5$ & - & - \\
\hline Start Time of Trip & Continuous & Time & - & - \\
\hline Schedule Deviation & Continuous & Seconds & 13.6 & 110.0 \\
\hline Boardings & Continuous & Passengers & 9.7 & 4.5 \\
\hline Alightings & Continuous & Passengers & 4.0 & 2.8 \\
\hline Onboard Load & Continuous & Passengers & 20.7 & 7.1 \\
\hline Dwell Time & Continuous & Seconds & 143.2 & 50.7 \\
\hline Average Speed & Continuous & Mph & 24.0 & 1.6 \\
\hline
\end{tabular}

The early/late variable is binary: 0 meaning early or on-time and 1 meaning late arrival to 
the "time point" at the start of the segment. If the buses arrive early, they are typically held at time points in order to adhere back to the schedule; however, if it arrives late, then it has extra slack time to simply move on to the next stop and get back on schedule. The time period variable is nominal representing the start time of each trip grouped together, 1: 4-6 AM, 2: 6-10 AM, 3: 10-4 PM, 4: 4-8 PM, 5: 8 PM-1 AM. This grouping allows a more focused analysis on different periods throughout the day. The start time variable is continuous, which is simply the actual start time of each trip. The total number of passengers boarding and alighting in between the segment are also input variables. The maximum number of passengers onboard during the segment is another variable. Maximum speeds are collected between each stop; the average inter-stop speed through the segment is used as a variable. Lastly, and possibly most importantly, the total dwell time through the segment (sum of the dwell times at the stops) is a variable as well also included in the analysis.

\subsubsection{Means Procedure}

Preliminary exploration of the data included analyzing the average travel time for the segment by time period as shown in Table 5 and Figure 35. Table 5 displays the number of observations for each time period. The table also includes the mean, standard deviation, minimum and maximum of segment travel time for each time period. Also, the average speed, in miles per hour, was calculated using the mean travel time for the 1.2 mile segment. This average speed may appear to be low, but it includes the dwell time as well. 
Table 3: Segment Travel Time Data Summary

\begin{tabular}{|c|ccccccc|}
\hline \multicolumn{7}{|c|}{ Analysis Variable : Segment Travel Time (seconds) } \\
\hline Period & N Obs & Time & Mean & St. Dev. & Minimum & Maximum & Avg. Speed \\
\hline 1 & 19 & $4-6$ & 349 & 40 & 285 & 402 & 12.4 \\
2 & 161 & $6-10$ & $\mathbf{4 1 5}$ & 50 & 285 & 534 & $\mathbf{1 0 . 4}$ \\
3 & 122 & $10-16$ & 405 & 54 & 280 & 553 & 10.7 \\
4 & 124 & $16-20$ & 403 & 61 & 255 & $\mathbf{5 6 6}$ & 10.7 \\
5 & 58 & $20-1$ & 328 & $\mathbf{6 6}$ & $\mathbf{2 0 5}$ & 514 & 13.2 \\
\hline
\end{tabular}

The AM peak period from 6-10 AM had the highest average segment travel time. Consequently, this peak period also had the lowest average speed. The late night period from 8 PM to $1 \mathrm{AM}$ had the lowest average segment travel time with the highest standard deviation. The variation of this time period may be explained by the higher traffic volumes and passenger movements from 8-10 PM compared to lower volumes and less passengers from $11 \mathrm{PM}$ to $1 \mathrm{AM}$. This data is presented in graphical form in Figure 35.

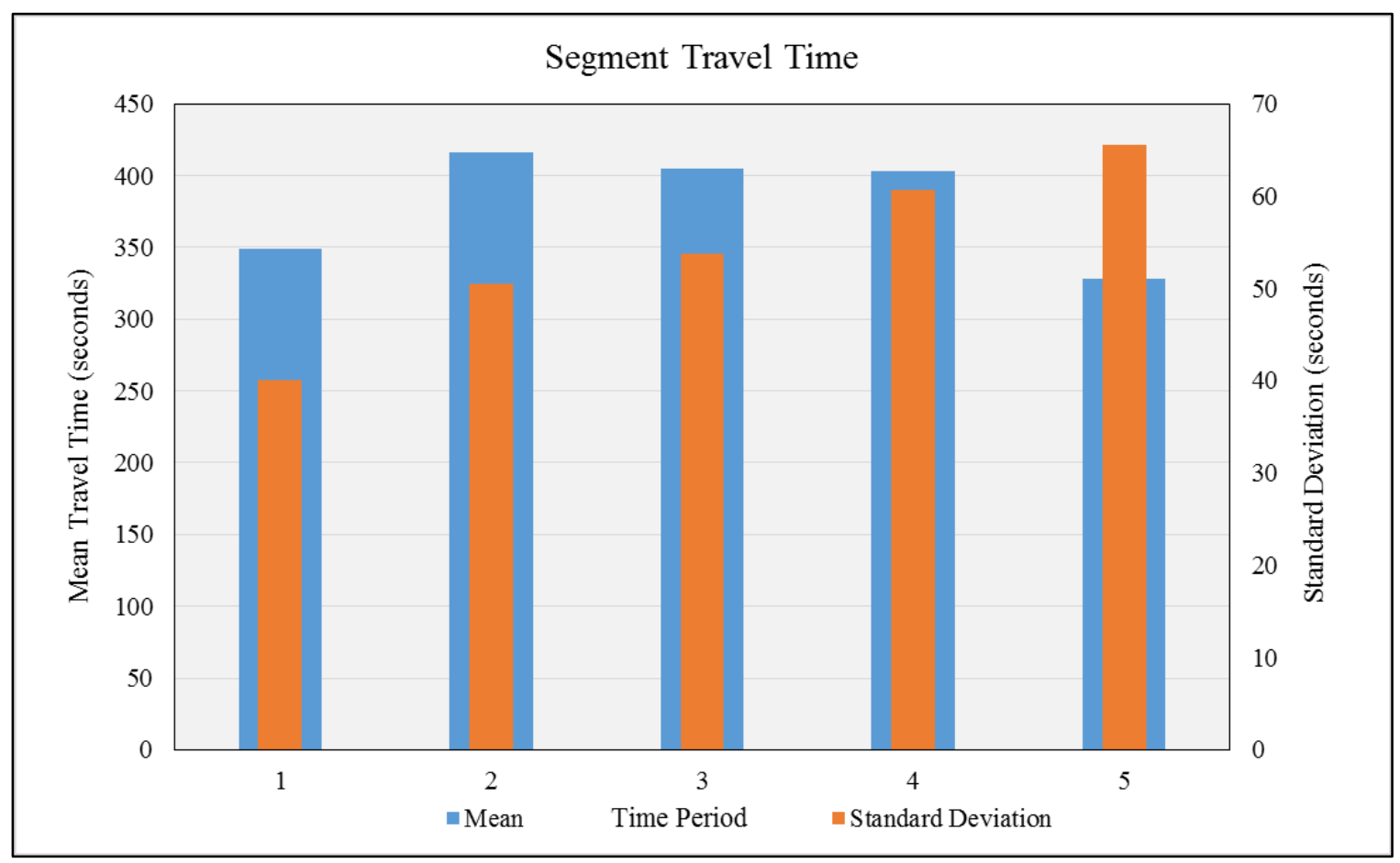

Figure 35: Segment Travel Time for each Time Period

The mean travel time is highest during the AM peak period 2, however it is fairly stable 
among periods 2, 3, and 4 (AM peak, mid-day and PM peak). The average travel times for periods 1 and 5 (early morning and late night) are much lower than the other periods. A reasonable assumption would be lower traffic volumes and passenger movements at those uncommon hours. The standard deviation is the lowest for the early morning period and highest for the late night period. The standard deviations are fairly stable for periods 2, 3 and 4 but the PM peak has slightly higher variability. A possible explanation for the highest standard deviation at the late night period would be the irregularity of traffic conditions and passenger movement: from 8-10 PM there are more passengers and higher traffic conditions, and from 10-1 AM there are much lower passenger movements and traffic conditions.

\subsubsection{Variable Correlations}

Another method to determine the effect of each variable included analyzing the Pearson Correlation Coefficients for all paired variable combinations. This measures the strength and direction of the linear relationship between two variables that is defined as the sample covariance of the variables divided by the product of their sample standard deviations. The closer the value is to +1 or -1 , the more closely the two variables are related. If the value is close to 0 , it means there is likely no relationship between the variables (random or nonlinear relationship). 


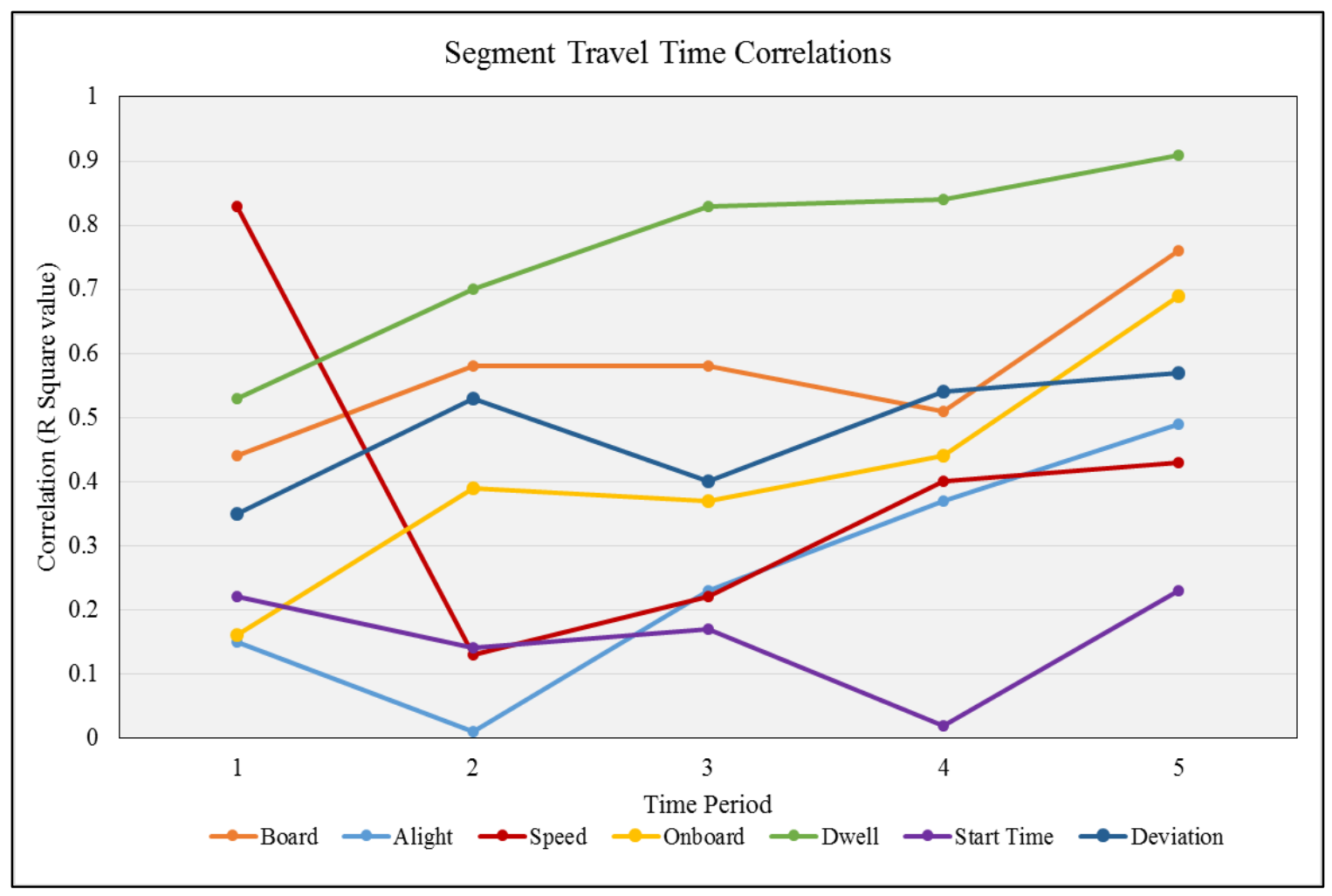

Figure 36: Segment Travel Time Correlations (Lines)

In order to compare how the variables are correlated based on the time period of the day, all correlations were taken as positive values for the line plot in Figure 36. However, the speed, alighting and start time variables with travel time were negatively correlated, which is presented in Figure 37. Figure 36 illustrates the changes in correlation of travel time with each variable across the five time periods. Although there are some weak relationships between a few of the variables, there are strong correlations with dwell time and average speed. Since this study is focused on the inbound trips, it is apparent that the alighting passengers have an extremely low correlation with travel time. Another explanation would be the time to alight is much lower than the time required to board the bus. It is surprising to see that all variables increase in correlation from period 4 to period 5, except for speed. From 8 pm to $1 \mathrm{am}$, there are fewer passengers and less traffic on the road so it would be expected that speed should be the main factor in travel 
time dependence. This may be explained by the time progression: around $8-9 \mathrm{pm}$ there is still traffic congestion on the roadways for those getting off work late, exploring the town, or grabbing a late dinner, but around midnight to 1 am there are two trips which probably have a couple of passengers and no traffic congestion at all.

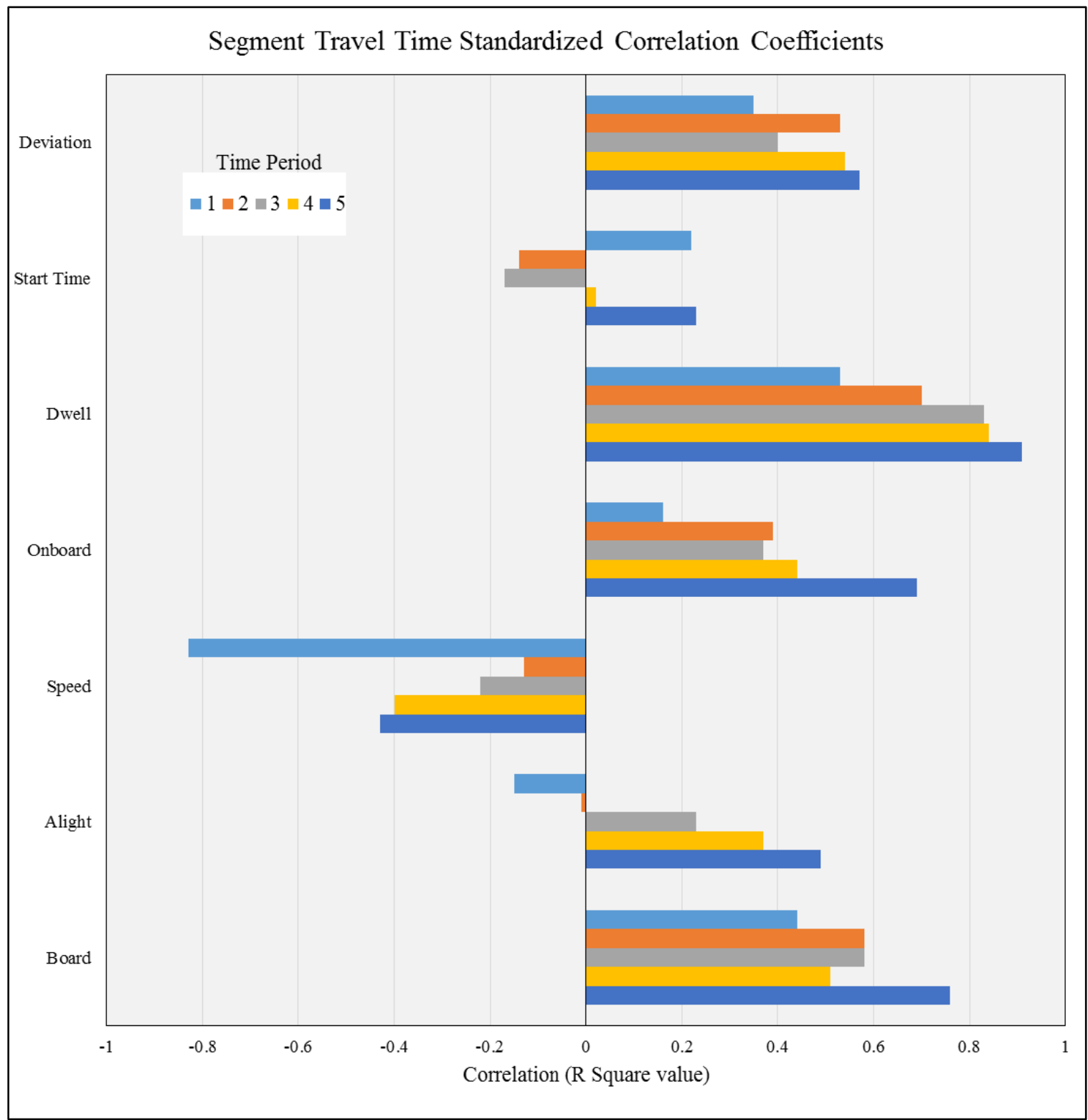

Figure 37: Segment Travel Time True Correlations (Bars)

Figure 37 illustrates how each time period is affected by the independent variables including negative correlations with speed, alightings and start times. Schedule deviation 
is a slight factor during periods 2,4 , and 5. Dwell time has a strong correlation with travel time regardless of time period, the weakest correlation is morning peak when there isn't much dwell time unless a bus arrives early. Start time has a weak relationship at all time periods. Onboard passenger load has the strongest correlation at the late night period. As mentioned previously, late nights include average size loads or no loads at all, which correspond with higher and lower travel times, respectively. Speed is highly correlated for early morning and late night. The negative correlation is expected because as the speed increases the travel time is expected to decrease. The early morning period is governed by speed, which is reasonable because there aren't many passengers or traffic conditions. Alighting is not correlated well at any time period, especially in the morning peak (all inbound boardings at that time). Boarding is most correlated with period 5, this is true because of lower boarding counts and lower travel times. The morning peak period is most correlated with boardings and dwell time, which go hand-in-hand. This trend continues for the mid-day and afternoon peak period. The late period is affected by several variables, which may need to be split into separate times. The regression and correlation results are presented in Appendix B. In order to determine the trends for the entire route, the outbound route would also need to be analyzed. The outbound route (from downtown center to southeast suburbs of Portland) would provide better correlations for alighting passengers.

\subsection{Decision Trees}

There are several ways to validate how well a model generalizes for observations not part of the data used to estimate the model, known as the training set. Decision trees were used for this purpose and were estimated with $70 \%$ (randomly drawn) data for 
training, and the remaining $30 \%$ used for validation. This decision tree model is used to attempt to explain the characteristics associated with the travel time of the segment. The variables explained above will be input, and the decision tree will help identify which variables are significantly affecting the travel time.

The first decision tree run includes all variables. The minimum leaf was set to 5 , which means that the tree will be created and split up until the last node has at least 5 data entries to validate. The classification tree resulting from these variables is provided in Figure 38 using 384 observations, 269 for training and for 115 validation. Each terminal node (last leaf of group) of the tree shown depicts the average of the travel time through the segment for that part of the leaf. It also shows the percentage of the data contained within that leaf in parentheses. The information provided in the root (initial) node represents the complete dataset. 


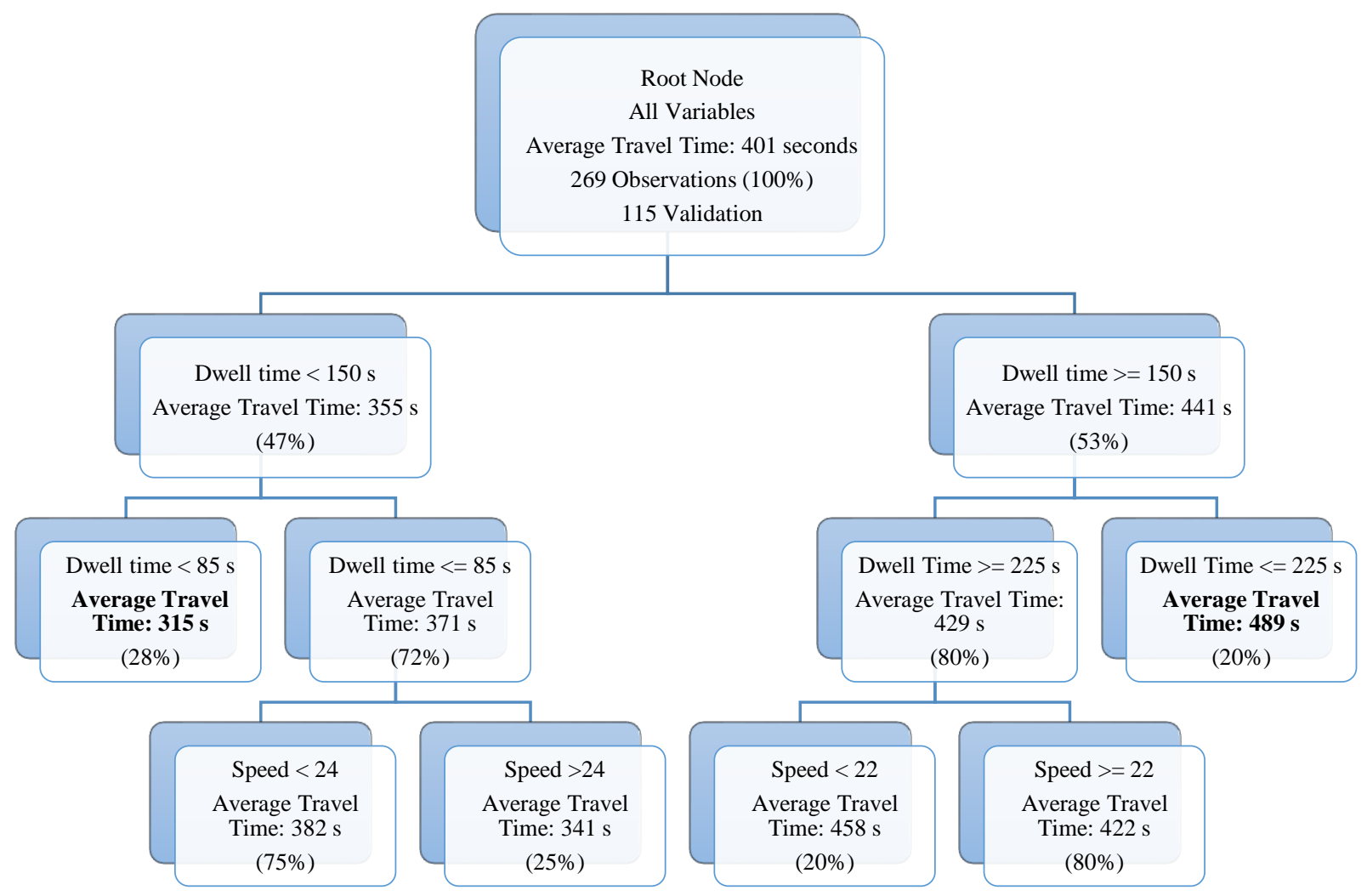

Figure 38: Decision Tree for Average Travel Time

When all of the variables are taken into consideration, dwell time and average speed were output as the most important independent variables. The average segment travel time for the initial root is $\mathbf{4 0 1}$ seconds, the validation from the dataset yields a lower average of 386 seconds. The first split of the data is based on the dwell time, whether it is greater than or less than 150 seconds. If the dwell time is greater than 150 seconds and also greater than 225, then the segment travel time is $\mathbf{4 8 9}$ seconds. If the dwell time is less than 150 and also less than 85 then the segment travel time is $\mathbf{3 1 5}$ seconds. As expected, the travel times decrease with lower dwell times and increase with higher dwell times. Additionally, if the dwell time is between 85 and 150 seconds and the speed is above 24 mph, the travel time is $\mathbf{3 4 1}$ seconds. Therefore, if there is a moderate level of dwell time 
and higher speeds, then the travel time will be much lower than the average. To examine other variables affecting travel time, dwell time was excluded in the next run.

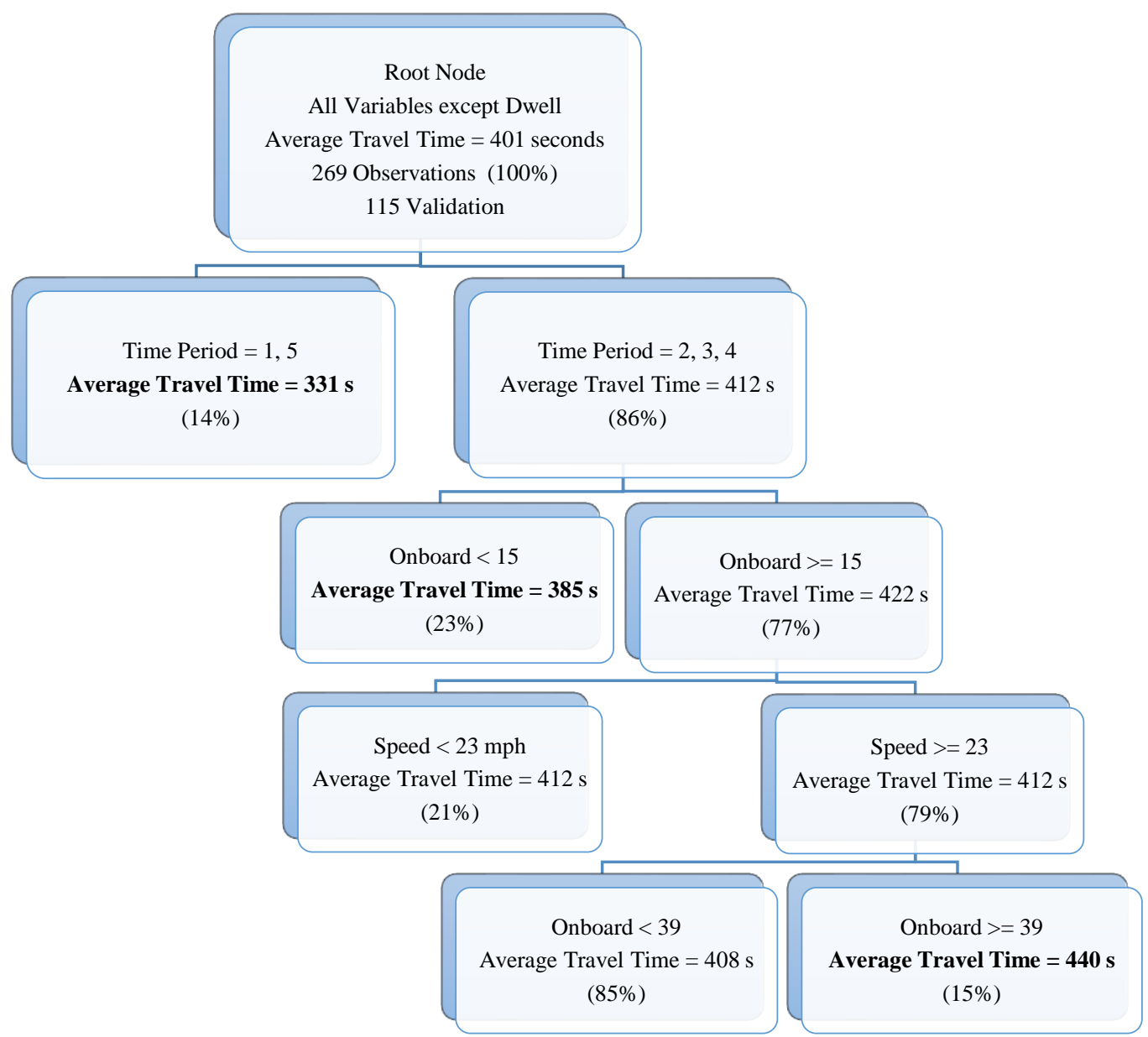

Figure 39: Decision Tree for Travel Time Excluding Dwell Time

The classification tree resulting from the remaining variables is provided in Figure 39, using the same 384 observations, 269 for training and 115 for validation. Removing the dwell time variable from consideration for the regression tree, the time period, onboard load and speed were the most important factors for travel time. If the period is 1 or 5 , then the segment travel time is only $\mathbf{3 3 1}$ seconds. This is understandable because of the lower passenger loads and traffic conditions in the early morning and late nights. However, at period 2, 3, or 4 and the onboard load is greater than 15 and the 
average speed is less than $22 \mathrm{mph}$, then the travel time is $\mathbf{4 5 4}$ seconds. This is also expected because at the morning, mid-day and afternoon peak periods, there are much higher passenger loads and worse traffic conditions causing delays and lower speeds along the route.

The third set of tree models excluded the dwell time and speed variables from consideration. Consequently, the decision tree resulted in the time period and onboard load as the most important variables remaining. However, these variables were not sufficient for the tree to make a reliable assessment on the travel time. The decision tree outputs are presented in Appendix C. The variables that significantly impact the trip time need to be incorporated into the trip time models. The dwell time was the most influential variable for travel time, which corresponds to the dwell time portion (at the stop) for the trip. Additionally, the average speed was the second most influential variable for travel time, which corresponds to the inter-stop portion (between stops) for the trip. 


\section{TRANSIT TRIP TIME MODEL}

Travel time is the duration of a passenger trip from the origin to the destination of the transit trip over a specified route. The travel time was calculated from layover stop at the beginning of the route to layover stop at the end of the route for the inbound direction, westbound. Travel time, reported as a time value, is closely tied to travel speed, reported as a travel rate. The conversion between travel time and travel rate is the distance between stops along the route. Travel time is of interest to the public, decision-makers, transit managers and transportation planners, as it is a performance measure understood by all. It is used to monitor service and measure passenger comfort (TCRP 88, 2003). Travel time is one of the key performance measures that needs to be monitored and maintained. With an entire month's worth of data it is possible to create and verify trip time models. The following sections will dissect the total trip time into dwell time and inter-stop time as described in the Literature Review and Newell Trip Time Model Data Preparation in Chapter 1.4.5 and Chapter 2.2.6, respectively. 


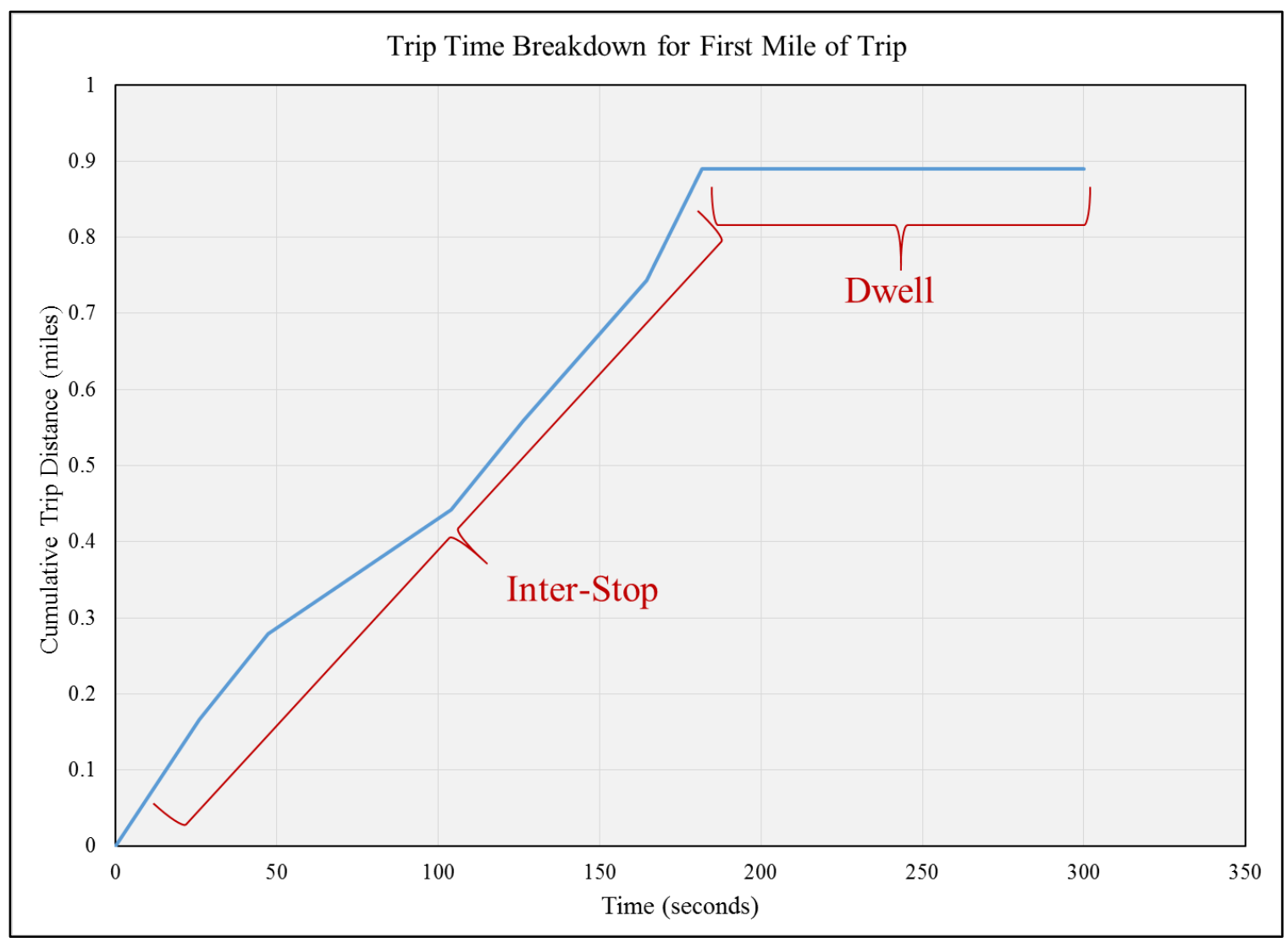

Figure 40: Inter-Stop Time vs Dwell Time for First Mile of Trip

Newell split inter-stop time and dwell time to analyze both of them separately (1995) as shown in previously in Figure 5. Figure 40, replicates the illustration from Newell using real data from the first mile segment (8 stops) of Route 14. The inter-stop portion varies in slope (different speeds) for each of the segments traveled. Additionally, the dwell time adds to the horizontal line, but each stop varies in passenger movements. Therefore, in order to create a transit trip time model, a model needed to be developed for dwell time and for inter-stop time separately.

\subsection{Dwell Time}

Dwell time is an important parameter that affects transit service quality (Levinson, 1983). Dwell time is the time the doors of the bus are open to allow passengers to board and or alight, which is a vital part of the trip time model. Originally 
the trip time model combined all of the passenger movements (boarding and alighting) for a simple linear regression model.

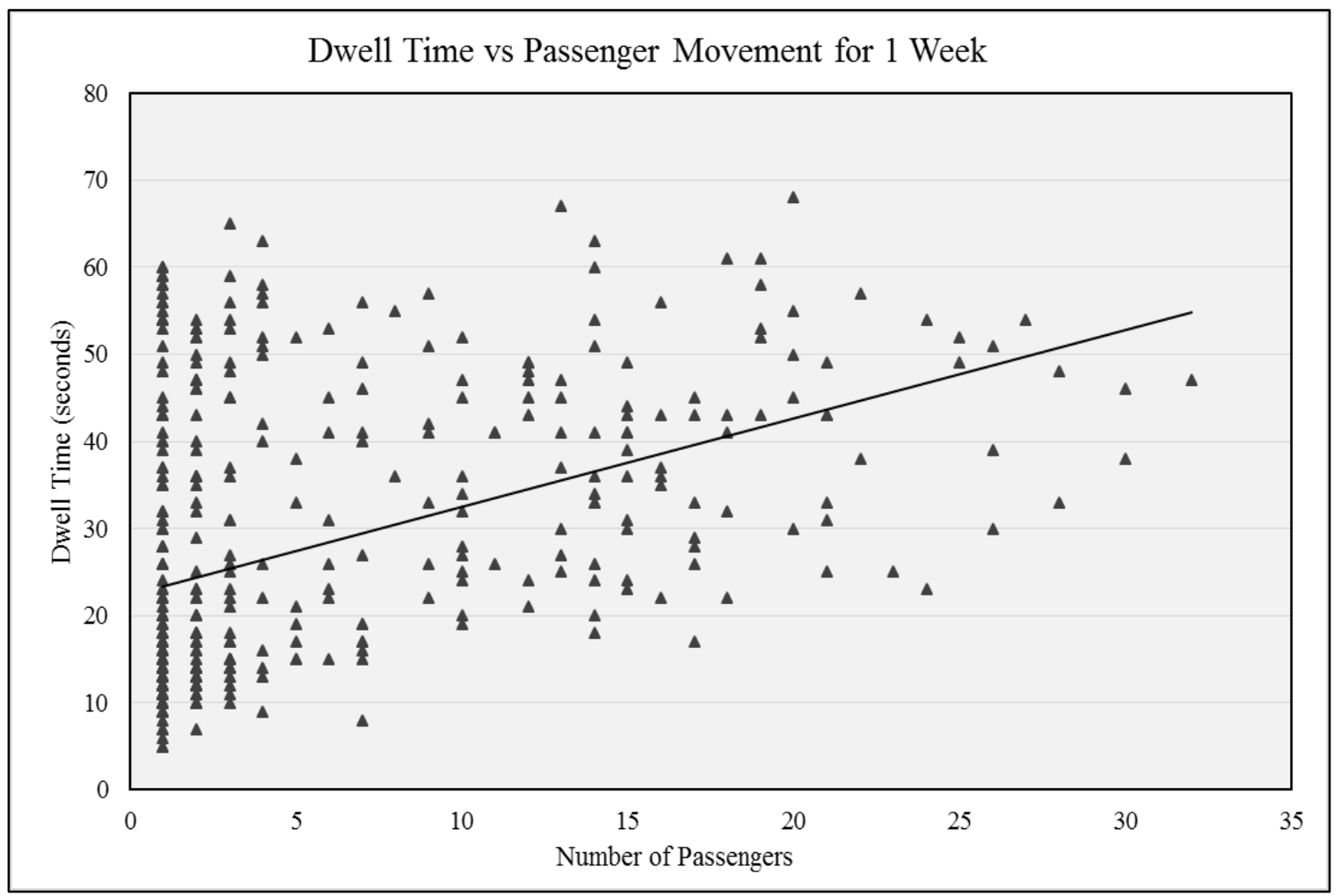

Figure 41: Dwell Time vs Passenger Movement for 1 Week

Figure 41 illustrates the original model: dwell time and passenger movement for all trips spanning from October 7-9, 2014. However, when alightings and boardings were combined, the regression output revealed a poor fit, R-squared value of 0.21 . Therefore, that model was discarded in favor of another model estimated using separate coefficients for the number of boarding and alighting passengers. Instead of using simple linear regression, a bivariate regression is used to measure the dwell for only alighting, only boarding, and for both, see Appendix D. This improved model incorporated how many times the bus actually stopped at a bus stop, the number of passengers that boarded and the number of passengers that alighted. 


\subsection{Inter-Stop Time}

Similar to dwell time, inter-stop time (travel time subtracted by dwell time) is also critical for the trip time. The next step was to investigate the relationship between interstop trip time and distance. The inter-stop time is the difference between the time the door was closed at the previous stop and the time the door was opened at the next stop. The inter-stop time is not a simple linear model, the slope (speed) varies between each stop. Figure 42 represents the inter-stop distances and times for the first mile of the trip.

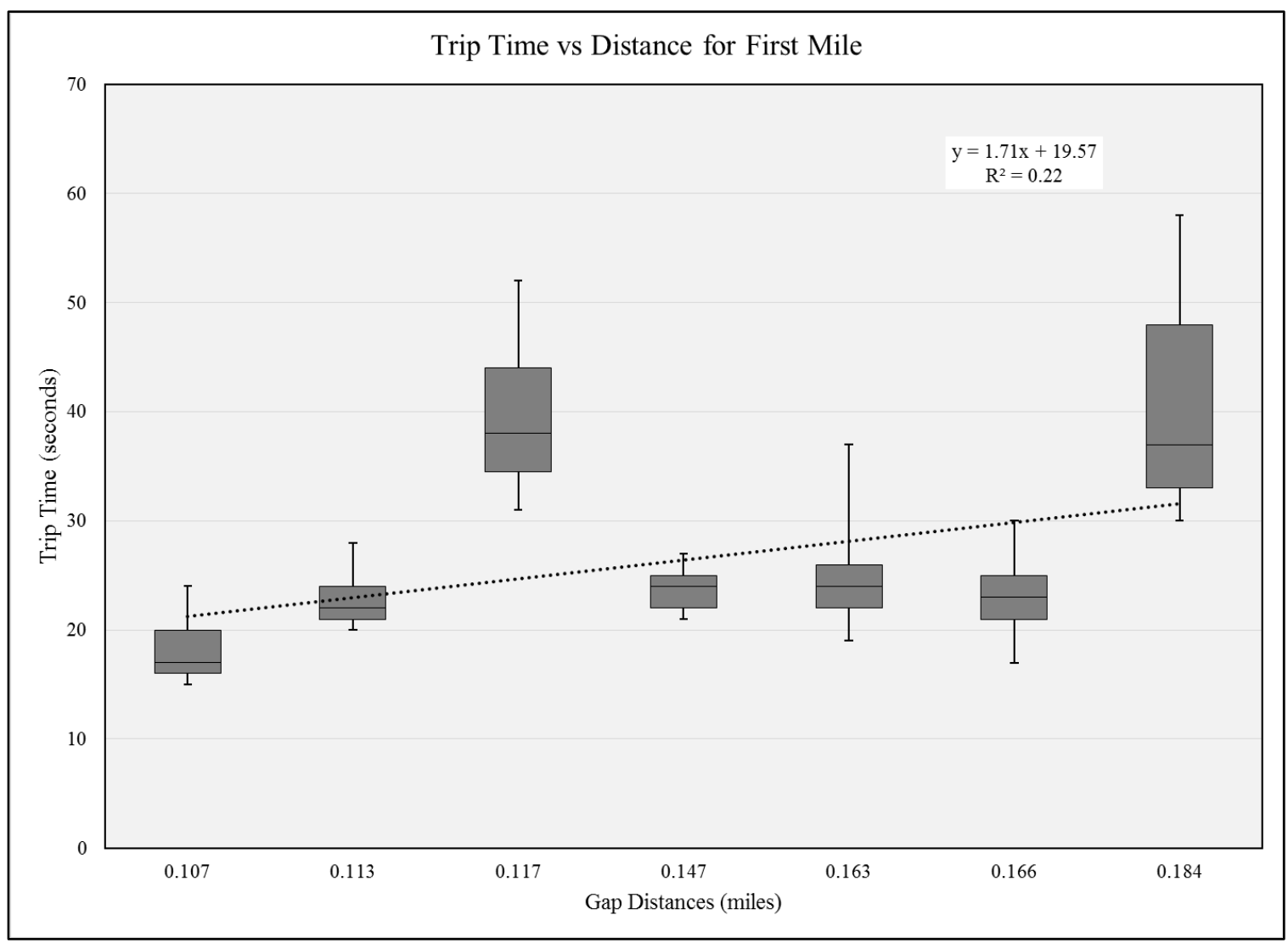

Figure 42: Boxplot of Trip Time vs Distance for First Mile of Trip

The boxplot emphasizes that the inter-stop time is not a linear average; the times are variable for each distance between consecutive stops. The segments with 0.117 and 0.184 miles difference had the highest variability; and the segment with 0.117 miles had the higher average inter-stop travel time even though it is nearly half of the distance. 
Although the inter-stop time varies between each stop, a simplified approach was taken by Newell (1995) for the trip time model.

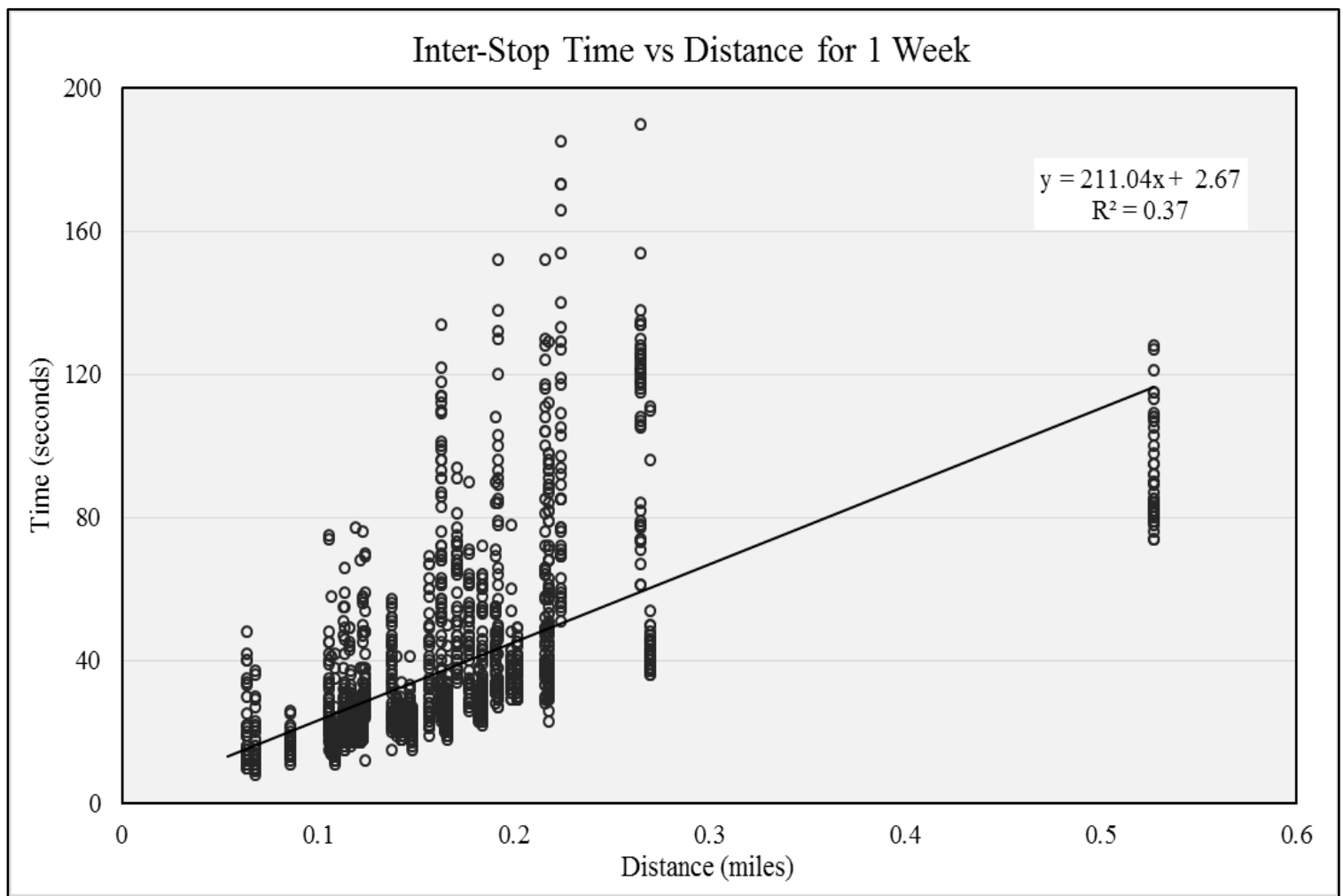

Figure 43: Inter-Stop Time vs Distance

Figure 43 represents the inter-stop trip times between consecutive stops. A simple linear regression was used to estimate a linear relationship between trip time in seconds and distance in miles. The reason that the majority of the points are vertical at various locations is because the location of the bus stops do not change. Most of the stops are spaced between 0.1 and 0.25 miles apart, except for two stops which are spaced about half a mile apart. This larger distance is from the bus crossing the Hawthorne Bridge. The model output confirms the graphical representation as:

$$
\text { Inter-Stop Time }(\text { seconds })=211.04 *(\text { distance })+2.67
$$

These results indicate that approximately 3 seconds of time lost is attributable to the deceleration and acceleration required for stopping. This value appears to be extremely 
low for the entire trip, but the amount of travel associated with each mile at 211 seconds appears to be reasonable compared to the 118.5 seconds for each kilometer, which corresponds to approximately 191 seconds per mile, from the Newell trip time model (Bertini \& El-Geneidy, 2004). The regression output revealed an R-squared value of 0.37 with parameters significant at the $95 \%$ level. Although the parameters were significant, the regression output does not have a strong correlation, this may be improved with the use of higher resolution data.

\subsection{Newell Trip Time Model}

There have been several methods to build a trip time model, splitting the run time and the dwell time were considered to be the best procedure. The dwell time and interstop time models are combined for a trip time model, defined below, built upon the methodology from Newell (1964), and further developed by Bertini and El-Geneidy (2004).

$$
T=T_{0}+a N_{d}+b N_{a}+c N_{b}
$$

$$
\begin{aligned}
& \text { Where } \quad T_{0}=\text { average inter-stop trip time } \\
& N_{d} \quad=\quad \text { number of dwells (stops) } \\
& N_{a}=\text { number of passengers alighting } \\
& N_{b} \quad=\quad \text { number of passengers boarding }
\end{aligned}
$$

This trip time model had statistically valid parameters that described the transit trip in a reasonable way, further illustrated in Appendix D. The next step was to further validate and test this model using 2014 archived data from the same Route 14 from TriMet. This is evident in Table 4, confirms that the previous trip time model is still relevant. 
Table 4: Newell Trip Time Model Summary for 2003 and 2014

\begin{tabular}{|l|c|c|}
\hline \multicolumn{1}{|c|}{ Model } & 2003 & 2014 \\
\hline \hline R Square & 0.4 & 0.37 \\
\hline Intercept & 26.0 & 19.33 \\
\hline ONS & 3.6 & 3.61 \\
\hline OFFS & 0.85 & 1.38 \\
\hline Units & $(\mathrm{km})$ & $(\mathrm{mi})$ \\
\hline Intercept & 20.2 & 2.67 \\
\hline Nonstop & 118.5 & 211.7 \\
\hline Mean Error & 0.05 & 0.03 \\
\hline
\end{tabular}

Although the model was developed using kilometers, it was created using the same length for the route, 7.2 miles or 11.6 kilometers. The mean error from the actual vs predicted values for trip times produced convincing numbers in 2003 and 2014. The Newell Trip Time Model is a good tool to predict transit trip times and impacts of various bus stops.

\subsection{New Inter-Stop Model}

The previous trip time model, similar to the majority of models, was deterministic. However, Hans et al. (2015) developed a combination of deterministic and probabilistic distributions for dwell time and trip time. The data used to calibrate this model was also archived from TriMet's database, along Route 72. There are two dwell time models, the first model uses dwell time to create a distribution for prediction, which is highly variable. The second model uses passenger movement to determine the amount of time spent at each stop, which the Newell Model fully covered and proved to be a strong predictor. Therefore, this model will focus on the inter-stop portion of the trip.

The inter-stop models that will be recreated use travel time distributions with archived transit data and signal locations. The models that were from Hans (2015) also included travel time based on synthetic and realistic trajectories with signal settings, velocity characteristics of buses and traffic flow from loop detectors. Although readily 
available, this advanced signal information was not used in the following analyses.

\subsubsection{Trip Time Model 1: Random Events}

A bus can be delayed by traffic signals, traffic flows and other random occurrences during its movement between two consecutive stops. In order to account for various delays in between stops, a 'random event' is added to the initial average speed inter-stop model. This variable is an addition to the average inter-stop time that can range from 0 seconds to 60 seconds. However, a random event may become negligible compared to delays occurring at signals. After discussions and further research into this type of random model behavior, this probabilistic model was not created.

\subsubsection{Trip Time Model 2: Signals}

Instead of considering all links to be the same, it is proposed to distinguish links without a traffic signal and with a traffic signal present. All of the 32 signalized intersections along Route 14 are depicted in Figure 10 in Chapter 2.1.1. The variability in inter-stop time is likely due to the effect of the delay superimposed by signals encountered. Signal delay is the main factor in varying inter-stop time (Hans et al., 2015). If the signals were consistent or if none were present, Newell's average inter-stop time model would be sufficient. The bus encounters signals at various times and locations throughout the day; therefore, this previous model is not susceptible to delay and needs to be enhanced.

All of the inter-stop segments were analyzed by subtracting the arrival time at the stop by the leave time at the previous stop. Each segment was analyzed for all trips: 45 stops producing 44 inter-stop segments. Table 5 represents individual inter-stop segments for the all trips for the work week of October 7-9, 2014. 
Table 5: Individual Inter-Stop Data Summary for 1 Week

\begin{tabular}{|c|c|c|c|c|c|c|}
\cline { 2 - 7 } \multicolumn{1}{c|}{} & \multicolumn{2}{c|}{ Inter-Stop Time } & \multicolumn{2}{c|}{ Distance (mi) } & \multicolumn{2}{c|}{ Speed (mph) } \\
\cline { 2 - 7 } \multicolumn{1}{c|}{} & Mean & St. Dev & Mean & St. Dev & Mean & St. Dev \\
\hline None & $0: 00: 27$ & $0: 00: 14$ & 0.140 & 0.037 & 20.6 & 5.7 \\
\hline Signal & $0: 00: 44$ & $0: 00: 31$ & 0.185 & 0.087 & 17.9 & 6.3 \\
\hline
\end{tabular}

As expected, the mean travel time for the inter-stop segments without a signal between stops was lower than the mean travel time for inter-stop segments with one or more signals in between stops. The majority of the segments with signals only had one signal, the only time there are segments with two signals are in the Portland downtown area. There are only three segments which have more than one signal, therefore, all segments with one or two signals were all grouped together. In addition to a higher average travel time, the standard deviation is also much higher than for segments without a signal. Although the segments with a signal are slightly longer segments, the average speed is a better component to compare because it incorporates the distance and time. Similarly, the average speed displays the same effect: segments with signals have a lower average speed and higher standard deviation.

Instead of building an extensive model to predict each inter-stop segment, the total inter-stop trip times were analyzed. Table 6 presents a summary of the total trip inter-stop times for each time period for the work week of October 7-9, 2014.

Table 6: Total Inter-Stop Trip Time Characteristics for 1 Week

\begin{tabular}{|l|c|c|c|c|c|}
\hline \multicolumn{5}{|c|}{ Inter-Stop Travel Time (seconds) Characteristics for 1 Week } \\
\hline Time Period & Mean & St Dev & Min & Max & Speed (mph) \\
\hline \hline AM & $\mathbf{1 6 6 9}$ & $\mathbf{1 5 7}$ & 1454 & $\mathbf{1 9 9 9}$ & $\mathbf{1 5 . 5}$ \\
\hline MID & 1575 & 129 & 1376 & 1878 & 16.5 \\
\hline PM & 1619 & 121 & 1473 & 1903 & 16.0 \\
\hline LATE & 1461 & 81 & $\mathbf{1 3 3 4}$ & 1589 & $\mathbf{1 7 . 7}$ \\
\hline ALL & 1600 & 143 & 1334 & 1999 & 16.2 \\
\hline
\end{tabular}

The average total inter-stop time is the highest for the AM peak period. This time period 
also has the highest standard deviation and maximum travel time. The late night period has the highest average speed Based on the summary outputs shown in Table 8, it appears that the variability in inter-stop travel time is highly dependent on whether or not the bus had to stop at a signal. Instead of relying on a 'random event' to add onto the trip time model, an inter-stop model with signal dependence needed to be created. After examining all of the signal locations along the bus route, it became apparent that it is not possible to create a model including signals using the BDS data. However, the higher resolution data provides further insight to what is occurring between stops. As shown in Figure 44, when the 5 second data has extended horizontal lines (at the same location for a period of time) it appears portray traffic signal delay. Therefore, it is possible to create a model based on the number of signals encountered for the entire trip.

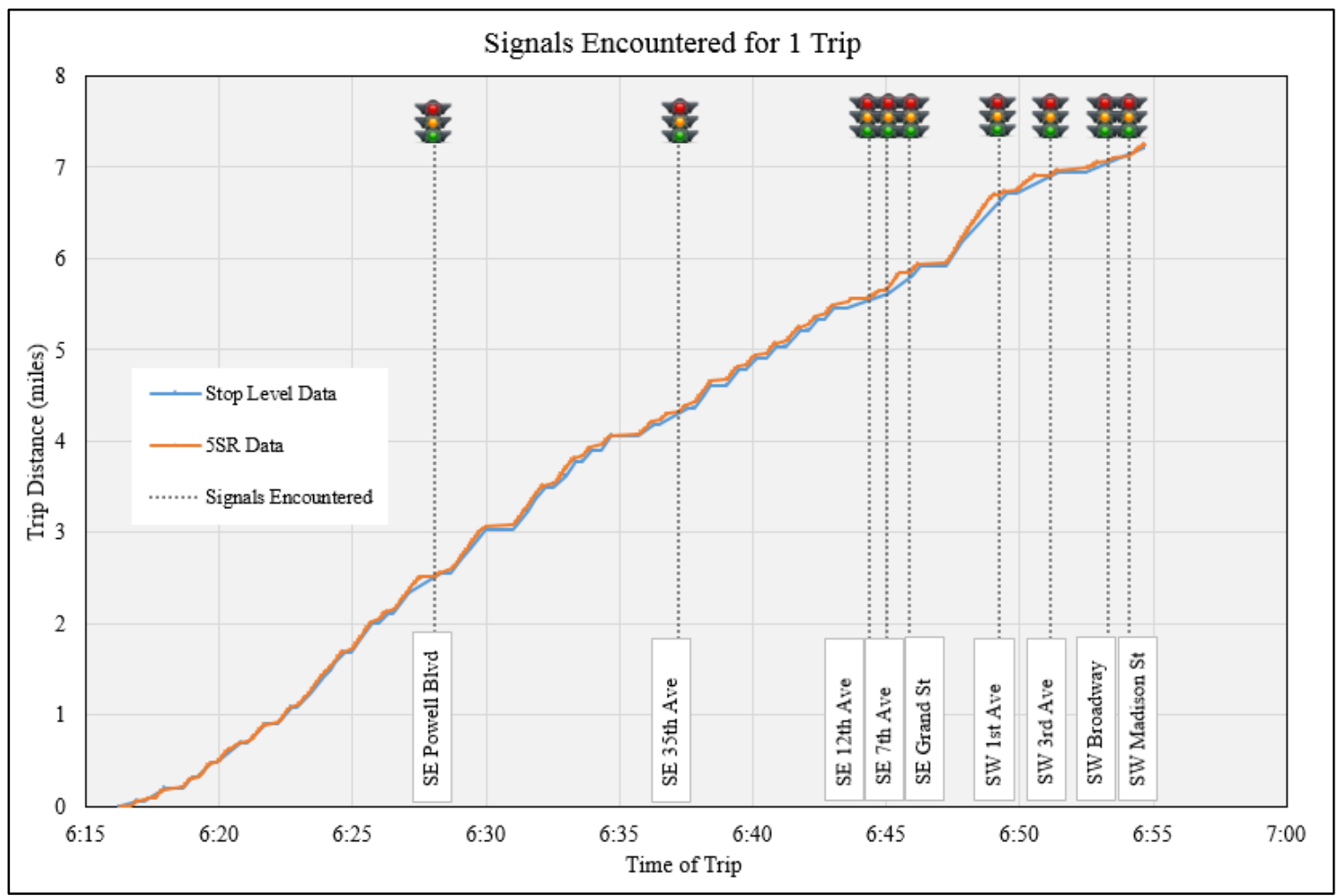

Figure 44: Signals Encountered for 1 Trip with BDS and 5SR

There are 9 signals encountered in Figure 44. As mentioned in Chapter 2.3.5, the 
5 second resolution data fluctuates above and below the stop level data. Figure 45 zooms into one of the segments from Figure 44 in order to further illustrate the higher resolution of the 5 second data.

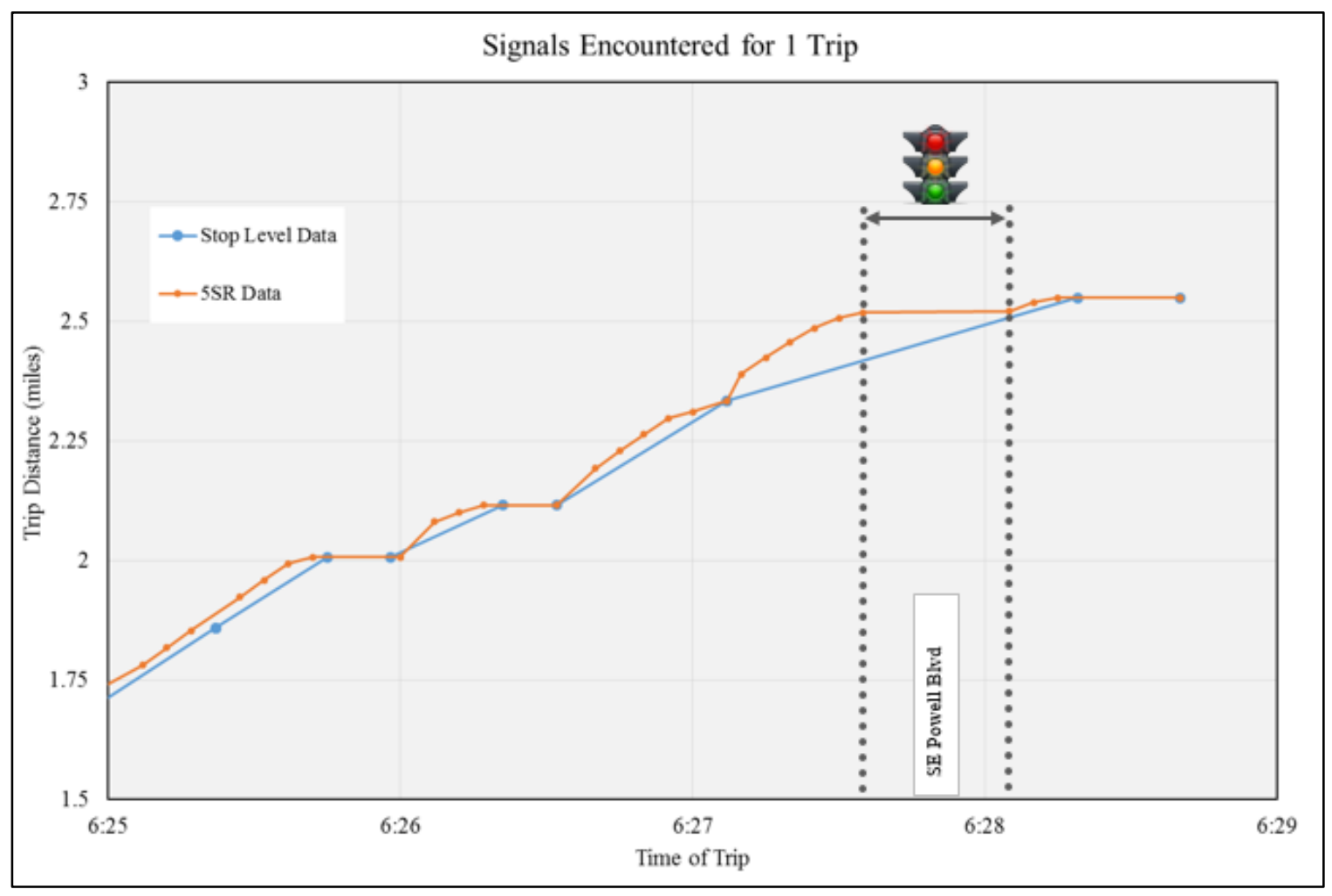

Figure 45: Signals Encountered for 1 Trip at SE Powell Blvd

A closer look allows the higher resolution data to stand out and identify locations of traffic congestion or signal delay. On Tuesday, October 7, 2014 at 6 AM a bus approached SE Powell Blvd to pick up passengers at the far-side stop. However, as shown by the 5 SR data, the bus had to stop and wait approximately 30 seconds waiting for the signal to change. Once the bus passed the intersection, it quickly stopped again in order to pick up the passengers at the bus stop, as shown by the horizontal dwell time. A better representation of the intersection and the far-side bus stop is shown in Figure 46 using Google Maps. As mentioned in the route description, the bus route crosses SE Powell Blvd, also known as US 26, is a major arterial that typically causes the bus to stop 
at the intersection before the bus stop.

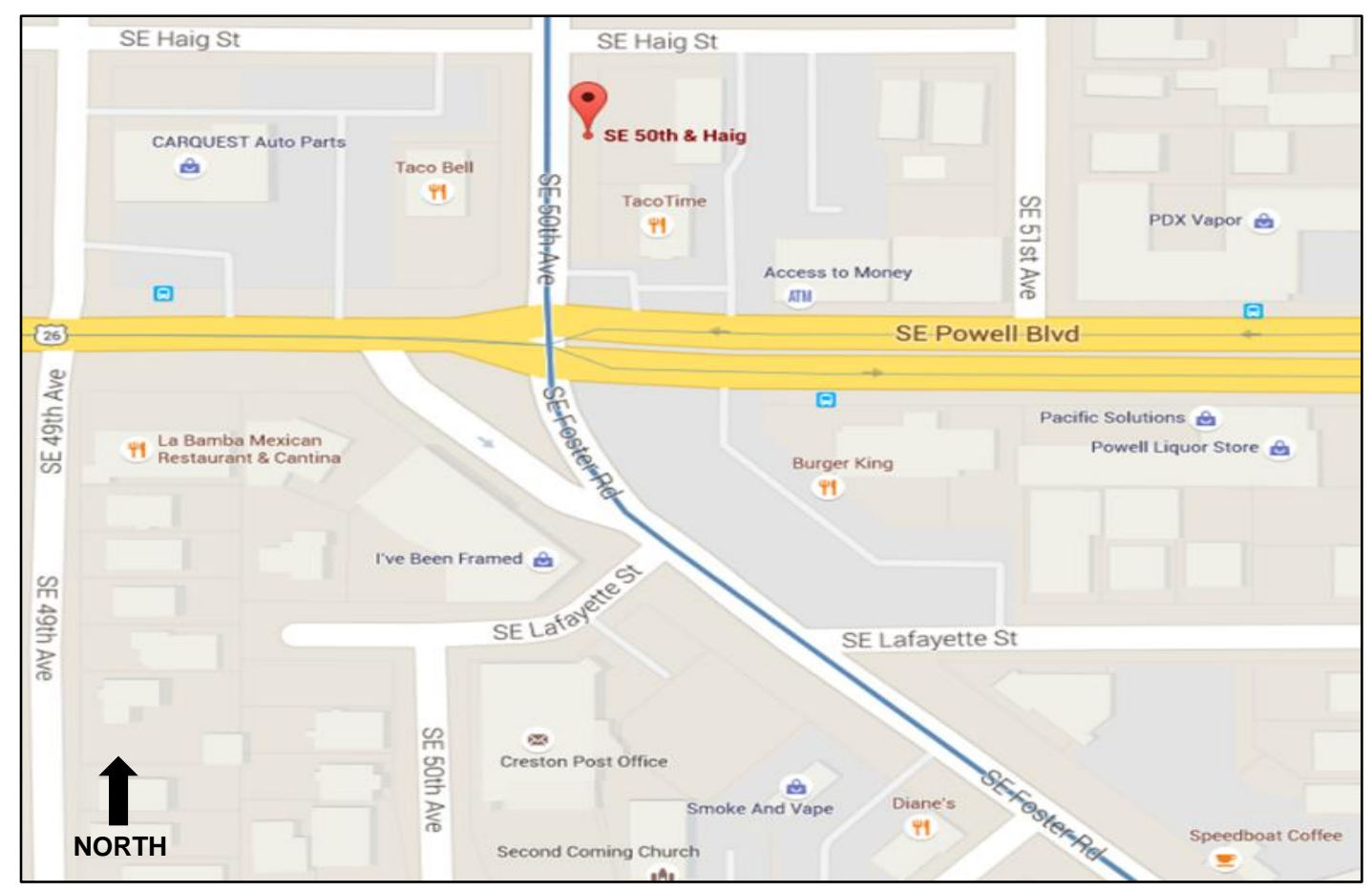

Figure 46: Google Maps Photo of Far-Side Bus Stop at SE Powell Blvd

The higher resolution data is able to determine whether or not the bus stops for a signal.

Stop level data must be used to distinguish between bus stopping for dwell time and stopping for a prolonged time outside of bus stops. A preliminary model was created to determine the linear relationship between signals encountered and inter-stop trip time. A sample of the methodology used to convert the 5-second resolution data to capture the number of signals encountered is shown in Appendix F. Figure 47 represents the linear relationship of signals encountered and total inter-stop time for an entire day, Tuesday, October 7, 2014. 


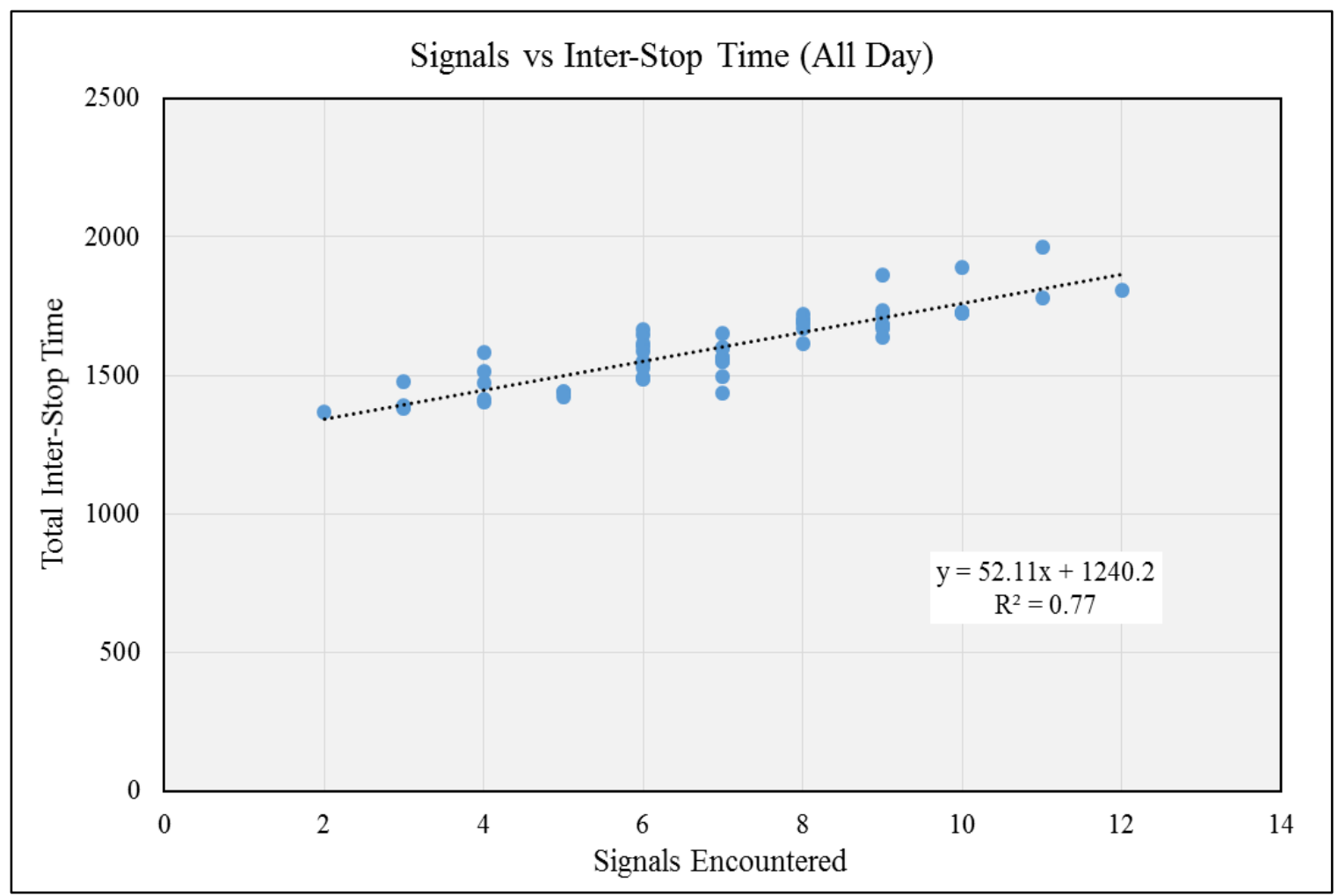

Figure 47: Signals Encountered vs Total Inter-Stop Time for All Day

There is a strong correlation as shown by the points on the line, and the $\mathrm{R}$ squared value. The linear regression equation suggests that each signal encountered adds approximately 52 seconds to the total inter-stop trip time. The equation also suggests that the inter-stop time is 1240 seconds if zero signals are encountered for the 7.2 mile route, which translates to about $21 \mathrm{mph}$.

In order to improve the model, signals and time period are taken into consideration for multivariate regression. Similar to creating correlations and decision trees in SAS, this software developed a regression model incorporating time period as a categorical variable and signals encountered as a continuous variable to forecast total inter-stop time. Figure 48 replicates the same model for signals encountered all day, but focuses on the AM peak period. 


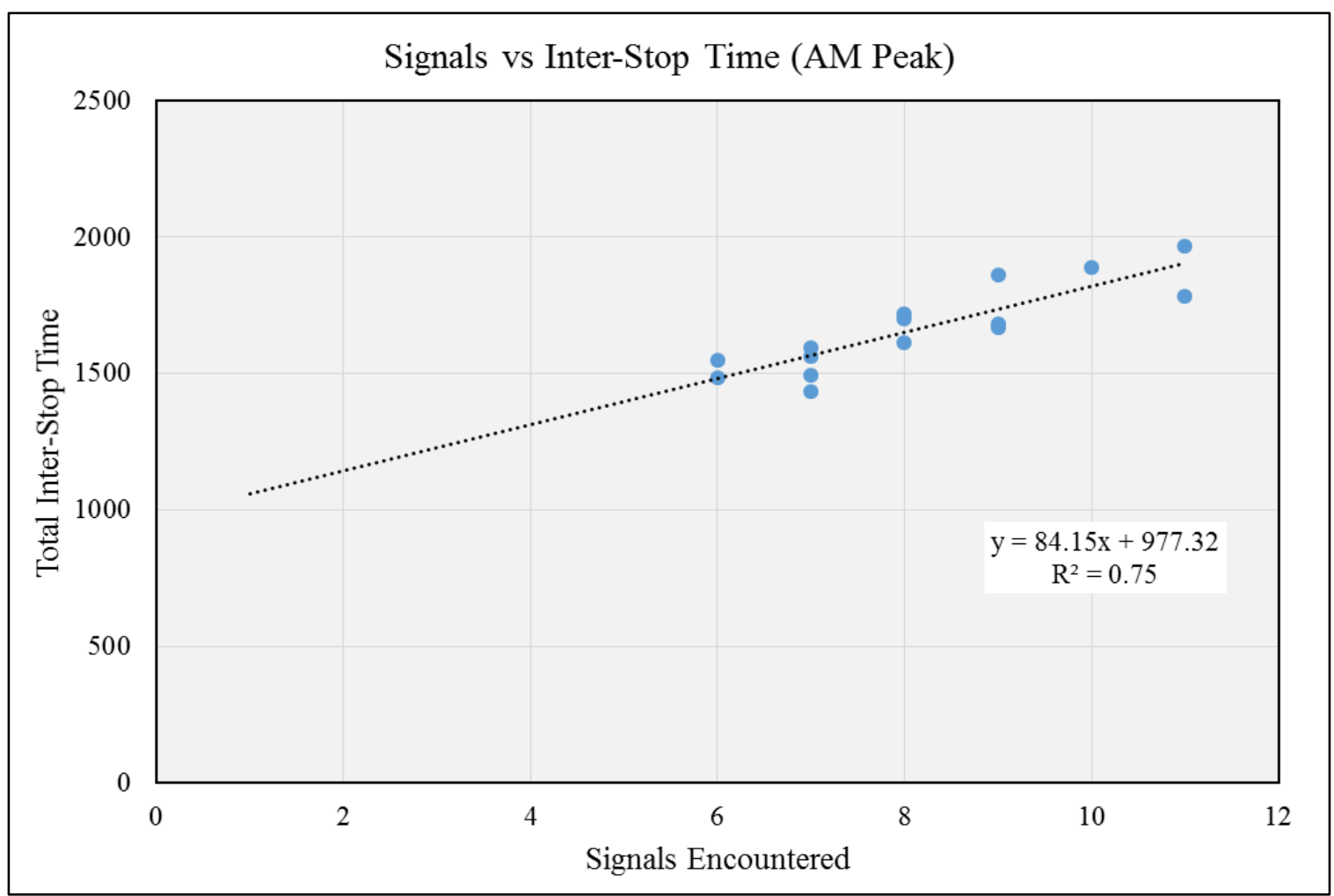

Figure 48: Signals Encountered vs Inter-Stop Time AM Peak Period

There is a very strong linear relationship between the signals encountered for the AM peak period, which matches the strength for the signals encountered all day. This procedure was repeated for all of the time periods. The linear regression models for each time period is shown in Table 7 and Appendix G, where the signal delay coefficient denotes the amount of time (seconds) each signal delays the trip.

Table 7: New Inter-Stop Trip Time Models

\begin{tabular}{|l|c|c|c|}
\hline Time Period & Intercept & Signal Delay Coefficient & R-Squared \\
\hline \hline AM & 977.3 & 84.2 & 0.75 \\
\hline MID & 1242.5 & 49.9 & 0.80 \\
\hline PM & 1334.4 & 40.8 & 0.76 \\
\hline LATE & 1287.9 & 41.1 & 0.64 \\
\hline
\end{tabular}

The formulas include an intercept for the inter-stop time if zero signals are encountered and a constant for the impact of each signal. The AM peak period has the lowest intercept, but makes up for the inter-stop time with the highest constant for each signal. 
The AM peak period is the most congested time period, with the majority of people traveling from the southeast suburbs into the Portland downtown center. The Late night period may not have the lowest intercept or constant, but it had a lower amount of signals encountered and ended up with the shortest inter-stop trip times. This new inter-stop trip time model will be compared to the previous Newell trip time model in the next section after validating the minimum sample size. 


\section{MODEL ROBUSTNESS}

No model will predict transit trip time with perfect accuracy. However, it is still important to develop and test trip time models based on different input variables. The Newell trip time model and the new inter-stop trip time model need to be assessed for their accuracy and statistical significance. This analysis of robustness has not been carried out in similar studies and may help in identifying standards for transit models. The decision tree analysis to determine which variables affected the travel time had validation built into the analysis. The following sections will discuss the minimum sample size requirements and the validity of the trip time models.

\subsection{Sample Size Validation}

For any travel time study, a minimum sample size is desired to verify the statistical significance of any results and to minimize the data collection cost in order to fit within budgetary constraints. Therefore, it is important to execute a number of travel time collection runs to determine a statistically permitted level of error from the sample (Bertini \& Tantiyanugulchai, 2004).

In general, the statistical estimation of the sample size $\mathrm{n}$ is based on specifying probability statements about the level of confidence in the error that is acceptable. The permitted error $\mathrm{E}$ is expressed as:

$$
E=Z_{\alpha / 2} \cdot \frac{\sigma}{\sqrt{n}}
$$

Where $\quad \mathrm{n}=$ minimum sample size

$Z_{\alpha / 2}=$ standard normal curve area to its right equals $\alpha / 2$ for a confidence level of $1-\alpha$

$\sigma \quad=$ standard deviation of population 


$$
\mathrm{E}=\text { maximum error of the estimation }
$$

Ranges of permitted errors in the estimate of the mean travel time are defined based on study purpose. Table 8 presents the permitted error based on the $95 \%$ confidence interval and the total number of observations used for the trip time model. The table also includes the permitted error for the minimum amount of data for the late night period. The permitted percent error is below $4 \%$, if the average total inter-stop trip time is a value of 1570 seconds, for total and minimum number of observations.

Table 8: Maximum Error Estimation at 95\% Confidence Intervals

\begin{tabular}{|l|c|c|}
\cline { 2 - 3 } \multicolumn{1}{c|}{} & Total & Minimum \\
\hline Confidence Interval & $95 \%$ & $95 \%$ \\
\hline Number of Observations & 64 & 12 \\
\hline Error in Seconds & 37.0 & 53.8 \\
\hline$\%$ Error/Total & $2 \%$ & $3 \%$ \\
\hline Error in Average Speed $(\mathrm{mph})$ & 0.5 & 1.0 \\
\hline
\end{tabular}

The table also includes the corresponding error in average speed (mph) based on the number of observations. For traffic operations, trend analyses or economic studies, a range of 2-4 mph is deemed acceptable (ITE, 2000). The percent error of total average ranges from $4-8 \%$ if the total average speed is a conservative value of $50 \mathrm{mph}$. Standards for trip time estimation and acceptable minimum errors have not been established. Therefore, a sensitivity analysis of varying errors of estimation and confidence levels were performed to produce a viable option to test for minimum sample size.

Often the estimation is done based on prior information or an initial sample (presample) which leads to a random variable having a t-distribution with $n-1$ degrees of freedom. At the same level of confidence of $(1-\alpha) 100 \%$, the new equation is written upon solving for n as (Quirogo \& Bullock, 1998): 


$$
n=\left[\frac{t_{a} \cdot s}{E}\right]^{2}
$$

Where $\quad \mathrm{s}=$ estimate standard deviation of random samples

$$
\begin{aligned}
t_{a}= & \mathrm{t} \text { distribution statistic (used instead of } \mathrm{Z} \alpha / 2 \text { when dealing with } \\
& \text { random samples or small sample size) } \\
\mathrm{E}= & \text { maximum error of the estimation }
\end{aligned}
$$

The sample size is based on specifying statements about the level of confidence in the error that is acceptable. The iterations to determine the minimum sample size requirement at $95 \%$ level of confidence is shown in Table 9. The iterations at the $90 \%$ level of confidence were removed since the requirements appeared to be met at the $95 \%$ level.

Table 9: Minimum Sample Size Requirement for 95\% Confidence Intervals

\begin{tabular}{|c|cccccc|}
\hline Iteration & Input $\mathrm{n}$ & $\mathrm{df}$ & t.95 & St. Dev. & Error & Output \\
\hline \hline 1 & 50 & 49 & 1.678 & 151 & 30 & 71.3 \\
2 & 71 & 70 & 1.667 & 151 & $\mathbf{3 0}$ & $\mathbf{7 0 . 4}$ \\
\hline 1 & 50 & 49 & 1.678 & 151 & 45 & 31.7 \\
2 & 32 & 31 & 1.696 & 151 & $\mathbf{4 5}$ & $\mathbf{3 2 . 4}$ \\
\hline 1 & 30 & 29 & 1.699 & 151 & 60 & 18.3 \\
2 & 18 & 17 & 1.74 & 151 & 60 & 19.2 \\
3 & 19 & 18 & 1.734 & 151 & $\mathbf{6 0}$ & $\mathbf{1 9 . 0}$ \\
\hline
\end{tabular}

The number of observations used in the analysis produces a maximum error of 37 seconds which is approximately $2 \%$ of the total inter-stop trip time. Alternatively, the minimum sample size for an error estimation of 30, 45 and 60 seconds requires 70,32 and 19 observations, respectively. In order to understand the table better, Figure 48 represents the maximum error based on the number of observations. 


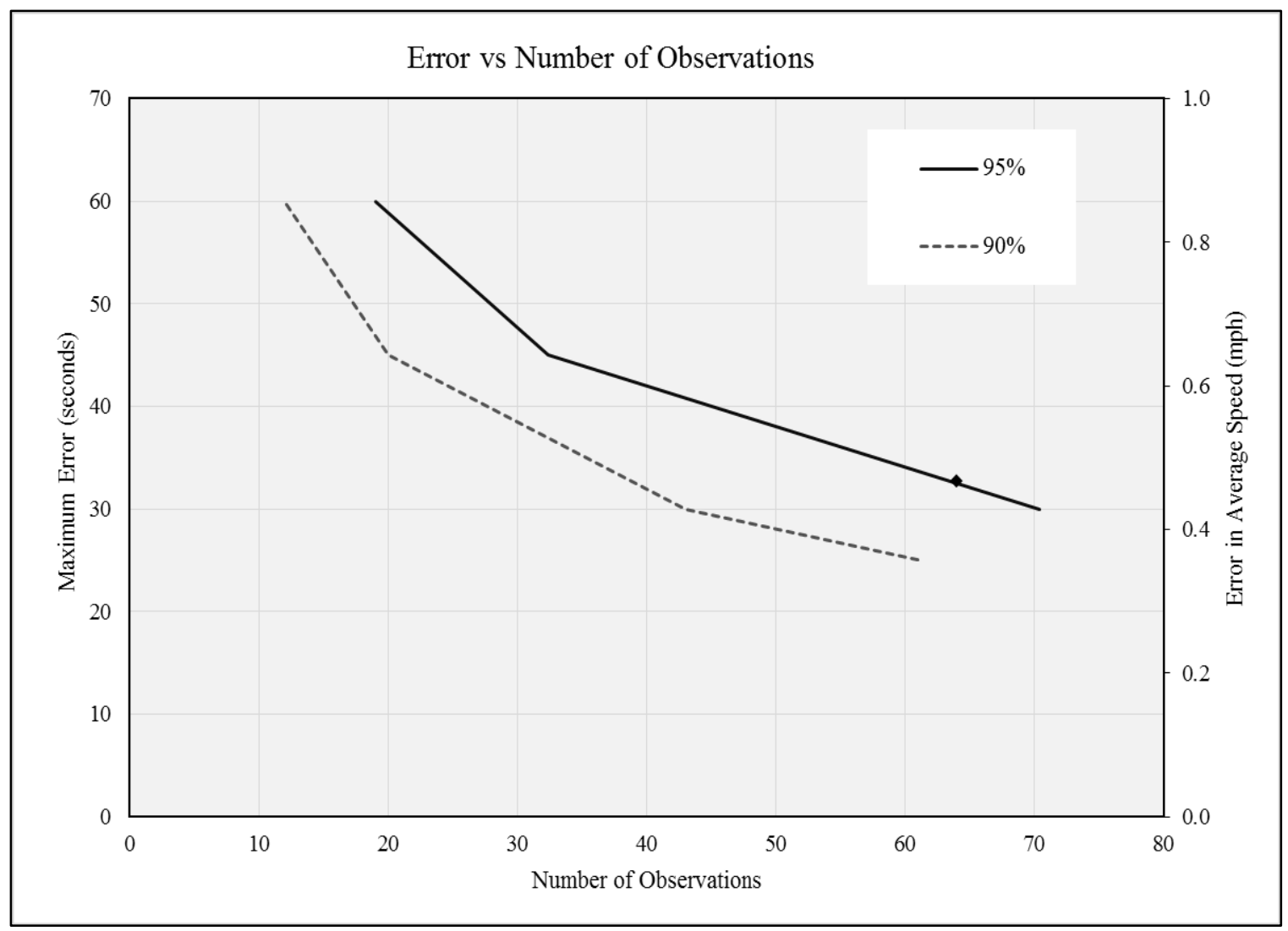

Figure 49: Minimum Number of Observations Based on Maximum Error

As the confidence level increases from 90 to $95 \%$ the minimum sample size requirements also increases. The point in Figure 49 represents the number of observations used in the analysis which corresponds to approximately 34 seconds of error estimation and $2 \%$ error of the total inter-stop time at the $95 \%$ confidence level. Also, this sample size corresponds to a $0.5 \mathrm{mph}$ error in average speed, which is acceptable and unlikely to be noticed by passengers.

\subsection{Model Comparisons}

The sample size and maximum error estimations met the desired requirements; therefore, a comparison and test of the models can be performed. A well-fitting regression model results in predicted values close to the observed data values. The mean model, which uses the mean for every predicted value, generally would be used if there 
were no informative predictor variables. Therefore, the fit of a proposed regression model should be better than the fit of the mean model; however, this must be proven through various statistical tests.

\subsubsection{Paired t-Test: Difference of Means}

This section uses the t-test difference of means to validate no difference between samples. To confirm the statistical validity of the relationship between predicted trip times and actual trip times, a hypothesis test concerning the difference of zero was conducted. The null hypothesis of $m_{1}-m_{1}=0$ was formulated to prove the existence of a relationship between predicted and actual values. This analysis was performed using:

- Alternative hypothesis: $m_{1}-m_{1} \neq 0$

- Level of significance: $\alpha=0.05$

- Number of sample pairs: $\mathrm{n}=52$

- T-critical: $\mathrm{t}_{0.025}$ for 51 degrees of freedom $= \pm 2.008$

Table 10: Paired T-Test for Newell and New Inter-Stop Model

\begin{tabular}{|l|r|r|r|r|}
\hline \multirow{2}{*}{ t-Test: Paired Two Sample for Means } & \multicolumn{2}{|c|}{ Newell Inter-Stop } & \multicolumn{2}{|c|}{ New Inter-Stop } \\
\cline { 2 - 5 } Mean & Actual & Predicted & \multicolumn{1}{c|}{ Actual } & Predicted \\
Variance & 1597.6 & 1570.0 & 1597.6 & 1603.6 \\
Observations & 22905.8 & 0.0 & 22905.8 & 25203.5 \\
Pearson Correlation & 52 & 52 & 52 & 52 \\
Hypothesized Mean Difference & - & & 0.896 & \\
df & 0 & & 0 & \\
t Stat & 51 & & 51 & \\
P(T<=t) one-tail & 1.313 & & -0.613 & \\
t Critical one-tail & 0.098 & & 0.271 & \\
\hline P(T<=t) two-tail & 1.675 & & 1.675 & \\
\hline t Critical two-tail & $\mathbf{0 . 1 9 5}$ & & $\mathbf{0 . 5 4 2}$ & \\
\hline
\end{tabular}

Paired t-tests for difference of means are used for both trip time models. Since the pvalue is 0.195 the null hypothesis cannot be rejected; therefore, the trip time model is 
statistically significant with a 95\% level of confidence. The New inter-stop model has a higher p-value which means the null hypothesis cannot be rejected either. Both trip time models appear to have similar results when compared to the actual trip times for the next work week, October 14-17, 2014.

\subsubsection{Regression Tests}

R-squared has the useful property that its scale is intuitive: it ranges from zero to one, with zero indicating that the proposed model does not improve prediction over the mean model and one indicating perfect prediction. Table 11 represents the summary output for the new inter-stop model. The parameters for this model are statistically significant at the $95 \%$ confidence level as the p-value is below 0.05 .

Table 11: New Inter-Stop Model Summary Output

\begin{tabular}{|l|c|c|c|c|}
\hline & Coefficients & Standard Error & t Stat & P-value \\
\hline \hline Intercept & 227.38 & 96.34 & 2.36 & $\mathbf{0 . 0 2 2}$ \\
\hline X Variable 1 & 0.85 & 0.06 & 14.29 & $2.759 \mathrm{E}-19$ \\
\hline
\end{tabular}

Improvement in the regression model results in proportional increases in $\mathrm{R}$ squared. One pitfall of R-squared is that it can only increase as predictors are added to the regression model. This increase is artificial when predictors are not actually improving the model's fit. To remedy this, a related statistic, Adjusted R-squared, incorporates the model's degrees of freedom. Adjusted R-squared will decrease as predictors are added if the increase in model fit does not make up for the loss of degrees of freedom. Likewise, it will increase as predictors are added if the increase in model fit is worthwhile. Adjusted R-squared should always be used with models with more than one predictor variable. It is interpreted as the proportion of total variance that is explained by the model. The adjusted R-squared value is displayed in Table 12. 
Table 12: New Inter-Stop Model Regression Statistics

\begin{tabular}{|l|c|}
\hline \multicolumn{2}{|c|}{ Regression Statistics } \\
\hline \hline Multiple R & 0.90 \\
\hline R-Squared & 0.81 \\
\hline Adjusted R-Squared & $\mathbf{0 . 8 0}$ \\
\hline Standard Error & 67.79 \\
\hline Observations & 52 \\
\hline
\end{tabular}

There are situations in which a high R-squared is not necessary or relevant. When the interest is in the relationship between variables, not in prediction, the R-square is less important. An R-squared in the range of 0.10 to 0.15 is reasonable for the situations as shown in previous figures. However, the adjusted R-squared value is strong for the new inter-stop model at 0.80 .

\subsubsection{Root Mean Squared Error}

Another statistical analysis includes the comparison of a calculated quantity known as the Root Mean Squared Error (RMSE). This is simply the square root of the mean square error. That is probably the most easily interpreted statistic, since it has the same units as the quantity plotted on the vertical axis. The RMSE is thus the distance, on average, of a data point from the fitted line, measured along a vertical line. The RMSE is directly interpretable in terms of measurement units, and a better measure of goodness of fit than a correlation coefficient. One can compare the RMSE to observed variation in measurements of a typical point. The two should be similar for a reasonable fit. The formula for RMSE is shown next.

$$
R M S E=\sqrt{\frac{1}{n} \sum_{i=1}^{N}\left(P_{i}-A_{i}\right)^{2}}
$$


Where

$$
\begin{aligned}
& \mathrm{N} \quad=\text { number of forecasted/observation pairs } \\
& \mathrm{P} \quad=\text { predicted trip time } \\
& \mathrm{A} \quad=\text { actual observed trip time }
\end{aligned}
$$

The RMSE is the square root of the variance of the residuals. It indicates the absolute fit of the model to the data-how close the observed data points are to the model's predicted values. Whereas R-squared is a relative measure of fit, RMSE is an absolute measure of fit. As the square root of a variance, RMSE can be interpreted as the standard deviation of the unexplained variance, and has the useful property of being in the same units as the response variable.

Lower values of RMSE indicate a better fit for the model. RMSE is a good measure of how accurately the model predicts the response, and is the most important criterion for fit if the main purpose of the model is prediction. The RMSE for the Newell and New Inter-Stop Models are $\mathbf{0 . 1 1}$ and $\mathbf{0 . 0 4}$, respectively. In order to have a graphical representation for the RMSE, the predicted values from both models were plotted against the actual inter-stop values in Figure 50. 

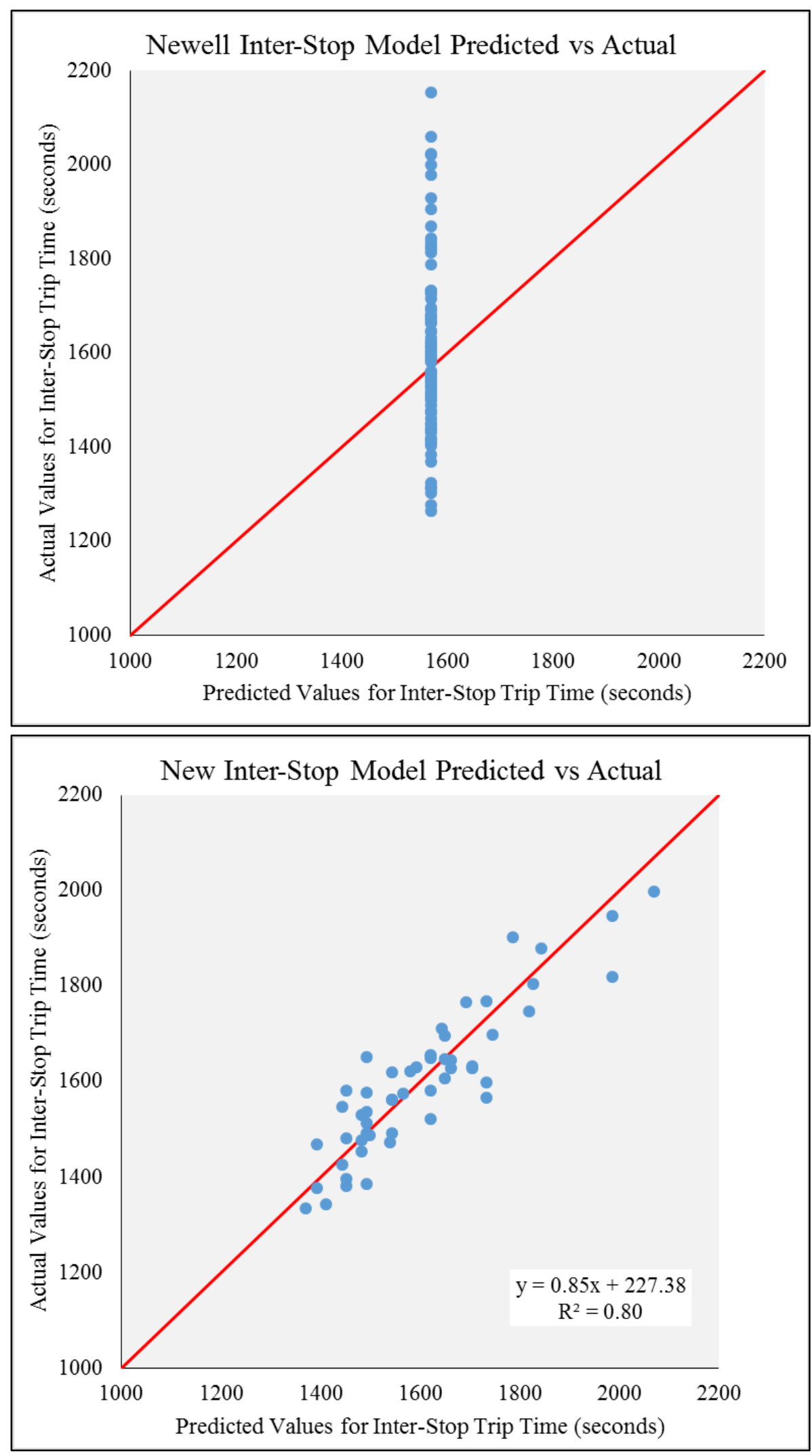

Figure 50: Predicted vs Actual Plot for Newell (Top) New Inter-Stop Model (Bottom) 
Although the Newell inter-stop model predicts an average inter-stop value, the RMSE was still fairly low, which interprets to an acceptable model. However, the RMSE for the new inter-stop model was extremely low and the parameters were statistically significant. This is further proven with the predicted times falling exceptionally close to the actual times. The difference between the actual inter-stop trip times and the predicted values produces the residual plot shown in Figure 51.

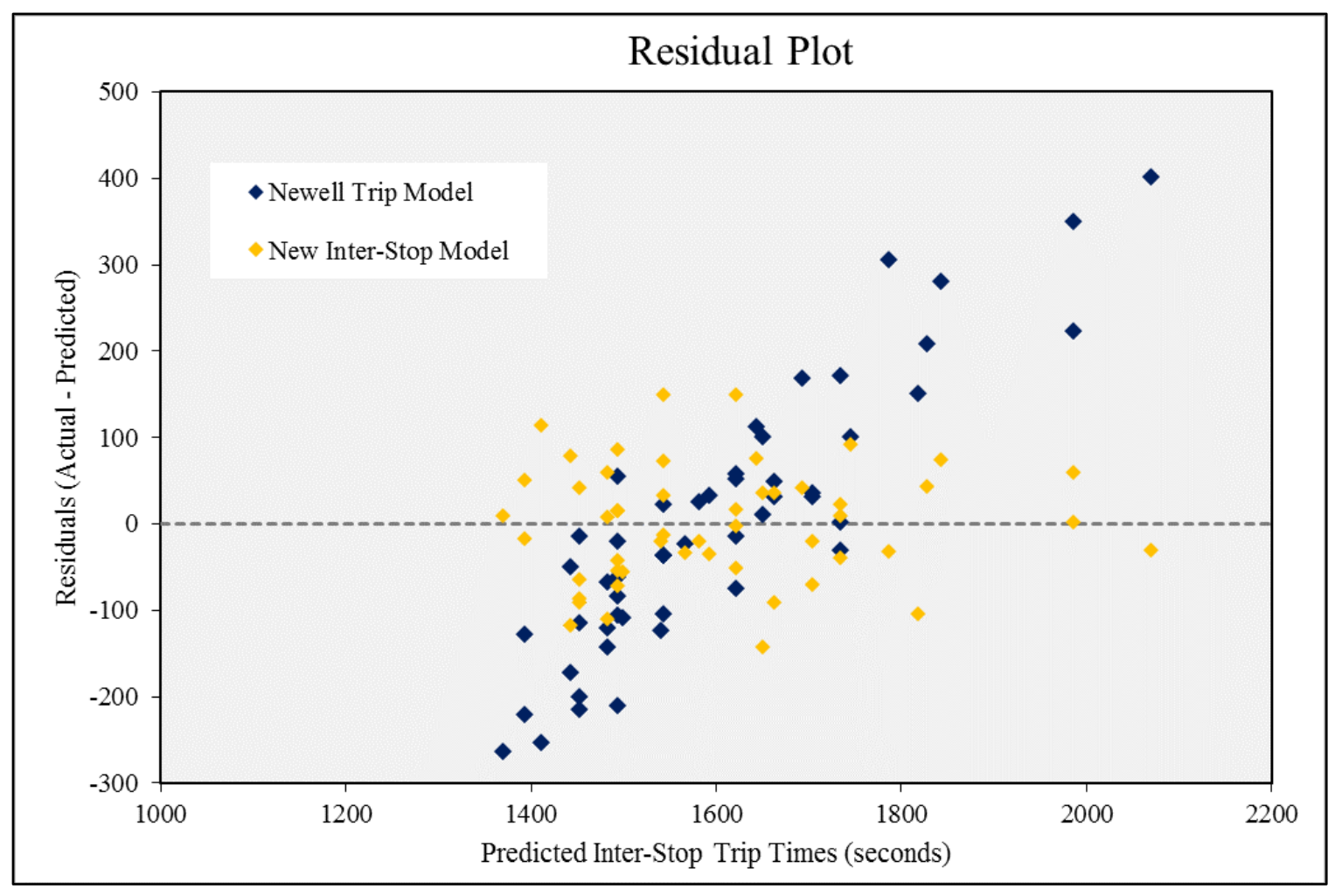

Figure 51: Residual Plot for Newell and New Inter-Stop Models

Since the Newell inter-stop model predicts an average inter-stop time for all trips, the residuals are dispersed. When the actual inter-stop time is low, then the Newell residuals are negative; subsequently, when the actual inter-stop time is high then the Newell residuals are positive. The New inter-stop model predicts lower and higher times, but the residuals are fluctuate near the dashed line for zero difference. As shown in the previous figure, the new inter-stop model is very accurate, there's a strong correlation 
between the models predictions and its actual results, further proven by the residual plot. Additionally, the random dispersion of residuals around the horizontal axis is preferred for a linear regression model.

\subsection{Data/Location}

The data used in the analyses is from one of numerous bus routes from TriMet in Portland, Oregon. Although the models are created for this region, they should be applicable to other areas as well. The peak periods may be altered to conform to the critical times needed in other locations. This higher resolution data may not be readily available in other locations, but if transit agencies have GPS tracking and AVL data collection on their fleet, this higher resolution data should be possible to set up. Of course this study may be limited by the data quality issues described previously. Also, some extreme (actual) values might have been eliminated from the datasets erroneously during the data cleaning stage. 


\section{CONCLUSIONS}

The objective of this research was to explore the potential benefits and applications of new higher resolution transit data on one transit route. This study analyzed various performance measures using both stop level and 5SR data. These analyses were performed using archived data from TriMet's inbound trip on Route 14 for October 2014. The inbound route typically has the highest usage, and lowest reliability because of the congestion from commuters.

This study used an observation data analysis based approach to assess the impact of several variables on transit travel time. The decision trees determined that dwell time and average speed between stops were the major factors influencing transit travel time. Subsequently, the Newell trip time model (Bertini \& El-Geneidy, 2004) was recreated using 2014 BDS data. This trip time model resulted in statistically significant parameters and proved to still be applicable. However, the Newell model took a simplified approach for the inter-stop portion of the trip by using a single value of average speed. The higher resolution data provides increasingly valuable and more highly granular information between stops. This data allows for more realistic conditions to be examined, and potential congestion and traffic conditions to be analyzed. The variability in inter-stop time is likely due to the effect of the delay superimposed by traffic signals and other traffic stream elements encountered. A new and improved inter-stop trip time model was developed based on the number of signals encountered during each time period. This model resulted in statistically significant results and provides more accurate predictions of inter-stop trip times.

When combined and applied, these trip time models can be valuable components 
of a transit agency's operations planning process. The techniques described in this paper are especially useful because they are all based on actual archived transit data. Trip time models can improve trip time reliability by understanding the variability of travel time along the route, which can attract more ridership. Trip time models can be used to assess operational improvements by identifying congested locations along the route. Transit agencies can reroute or reschedule the timings of the route based on these models. Additionally, trip time models may also be used for evaluating bus stop consolidation or other changes in service. The goal for transit agencies is to minimize operational costs and maximize accessibility for users. Each additional stop added along the trip adds to the total trip time. The time saved solely due to acceleration and deceleration at a stop was estimated as 17 seconds per stop (Li \& Bertini, 2009). The higher resolution data can be utilized to determine more accurate estimates of acceleration and decelerations around bus stops. Also, it should be noted that the 5 -second resolution data needs to be combined with the stop-level BDS data to compare and extract the data and provide passenger movement information.

\subsection{Future Recommendations}

As with any observational data mining method; this study could benefit from a larger sample size. The minimum sample size requirements were met, but a larger sample size could be used for each of the analyses performed. This could include more days, directions, routes, and segregation according to weather or season. The methods used in this thesis should be easily transferable to other locations where archived transit data is implemented.

Future applications of the higher resolution data could include the use of artificial 
neural networks to improve trip time models, identify bus bunching events, model gaps between consecutive buses on the same route, and analyze impacts of intersections with various weather conditions. The difference in travel speeds and congestion along the route can be compared during rainy days with the higher resolution data. There is possibly lower transit ridership and more drivers on these days causing more delay. The impacts of intersection traffic control devices can be determined between stops, which include signals with transit signal priority. Traffic signal data was not used in this study, but it is possible to use these trip time models to demonstrate and test the operational impact of traffic signal system improvements (Hans et al., 2015). Pairing this higher resolution data with the SCATS signal phase log data collected at intersections (Feng, 2014) would provide great opportunities to better understand the queuing effect on bus travel time and evaluate TSP system performance. Also, as bus bunching is inevitable, this higher resolution data can be used to monitor the interactions between buses once the phenomenon occurs. Previous schedule recovery processes can be tested using the higher resolution data (Lin \& Bertini, 2004).

Similar to Glick et al. (2015) these analyses call for a recommendation for a change in TriMet's 5SR data structure and archiving parameters. It is highly recommended that reports should be made every 5 seconds regardless of bus motion. Ideally the 5SR data should be time-synched with the stop level data and should report at even 5 second increments. This will allow for accurate stop times and the locations of these stops to be directly analyzed. Currently, only an assumption of slow speed can be used and actual stopping time is uncertain. In addition, it should be noted that without a few additional pieces of information, 5SR data is not accessible on its own. It requires 
that stop level data be used to compare and extract the data. This could be resolved by including additional fields in the data about train and trip number. Wheel sensor movement data could be another complementary dataset to overcome these limitations.

From this study, it is suggested that future research should also be conducted during different peak periods on this study corridor or expanded into other corridors with different characteristics and different bus routes such as, in Portland, Division Street (Route 4), Powell (Route 9) and Burnside Street (Route 20). Incident information should also be taken into account for further analysis including a study using non-linear regression in creating a travel time model. Such results will provide a more robust understanding of what occurs between stops and estimating arterial traffic conditions. 


\section{REFERENCES}

Bertini, R., \& El-Geneidy, A. (2003). Generating Transit Performance Measures with Archived Data. Transportation Research Record: Journal of the Transportation Research Board, 1841: 109-119.

Bertini, R., \& El-Geneidy, A. (2004). Modeling Transit Trip Time Using Archived Bus Dispatch System Data. Transportation Research Record: Journal of the Transportation Research Board, 1842: 56-67.

Bertini, R., \& Tantiyanugulchai, S. (2004). Transit Buses as Traffic Probes: Empirical Evaluation Using Geolocation Data. Transportation Research Record: Journal of Transportation Research Board, 1870: 35-45.

Dueker, K., Kimpel, T., Strathman, J., \& Callas, S. (2004). Determinants of Bus Dwell Time. Journal of Public Transportation, 7(1): 21-40.

Feng, W. (2014). Analyses of Bus Travel Time Reliability and Transit Signal Priority at the Stop-To-Stop Segment Level. Dissertations and Theses. Paper 1832.

Feng, W., \& Figliozzi, M. (2011). Using Archived AVL/APC Bus Data to Identify Spatial-Temporal Causes of Bus Bunching. In Proceeding of the $90^{\text {th }}$ Transportation Research Board Meeting, (No. 11-4262).

Furth, P., Hemily, B., Muller, T., \& Strathman, J. (2006) TCRP Report 113: Using Archived AVL-APC Data to Improve Transit Performance and Management. Transportation Research Board of the National Academies.

Gayah, V. Yu, Z., \& Wood, J., (2016) Modeling Bus Travel Times and Travel Time Uncertainty: Comparison of linear and Survivor Model Frameworks. In Proceeding of the $95^{\text {th }}$ Transportation Research Board Meeting, (No. 16-0769). 
Glick, T. (2015). Using High Resolution Archived Transit Data to Quantify Congestion at Intersections of Urban Arterials. Portland State University, Honors Theses, Paper 147.

Glick, T., Feng, W., Bertini, R., \& Figliozzi, M. (2015). Exploring Applications of Second Generation Archived Transit Data for Estimating Performance Measures and Arterial Travel Speeds. Transportation Research Record: Journal of Transportation Research Board, 2538: 44-53.

Hans, E., Chiabaut, N., Leclercq, L., \& Bertini, R. (2015). Real-Time Bus Route State Forecasting Using Particle Filter and Mesoscopic Modeling. Transportation Research Part C: Emerging Technologies 61, 121-140.

Highway Capacity Manual. (1985). HCM Special Report 209. Transportation Research Board, 12-3.

Institute of Transportation Engineers. (2000). Manual of Transportation Engineering Studies, 55.

Koffman, D. (1978). A Simulation Study of Alternative Real-time Bus Headway Control Strategies. Transportation Research Record: Journal of Transportation Research Board, 663: 41-46.

Langston, J. (2014). 3 Charts: Bus Cuts Drive Riders Away. Sightline Institute. Retrieved April 15, 2016, from: http://www.sightline.org/2014/03/17/3-charts-bus-cuts-driveriders-away/

Levinson, H. (1983). Analyzing Transit Travel Time Performance. Transportation Research Record: Journal of Transportation Research Board, 915: 1-6.

Li, H., \& Bertini, R. (2009). Assessing a Model for Optimal Bus Stop Spacing with High- 
Resolution Archived Stop-Level Data. Transportation Research Record: Journal of Transportation Research Board, 2111: 24-32.

Lin, W., \& Bertini, R. L. (2004). Modeling Schedule Recovery Processes in Transit Operations for Bus Arrival Time Prediction. Journal of Advanced Transportation ATR, 38(3): 347-365.

Meng, Q., \& Qu, X. (2013). Bus Dwell Time Estimation at Bus Bays: A Probabilistic Approach. Transportation Research Part C: Emerging Technologies, 36: 61-71.

National Center for Transit Research (2005). Enhancing the Rider Experience: The Impact of Real-Time Information on Transit Ridership. Center for Urban Transportation Research, University of South Florida.

Newell, G. (1995). Lecture Notes on Public Transportation. Department of Civil Engineering, University of California, Berkeley.

Newell, G., \& Potts, R. (1964). Maintaining a Bus Schedule. In Proceedings of $2^{\text {nd }}$ Australian Road Research Board, 2: 388-393.

Park, T., Nuworsoo, C., \& Pande, A. (2015). Preliminary Demand Analysis Framework for Bike-Sharing Program through Exploration of Information from the Census Bureau Data. In Proceeding of the $95^{\text {th }}$ Transportation Research Board Meeting, (No. 16-4993).

Portland Traffic Counts. (2014). Portland Bureau of Transportation, City of Portland, Oregon.

Quiroga, C., \& Bullock, D. (1998). Determination of Sample Sizes for Travel Time Studies. Institute of Transportation Engineers (ITE) Journal, 64: 92-98.

Robinson, S. (2013). Measuring Bus Stop Dwell Time and Time Lost Serving Stop with 
London iBus Automatic Vehicle Location Data. Transportation Research Record: Journal of Transportation Research Board, 2352: 68-75.

Shaaban, K., \& Pande, A. (2015). Analysis of Observational Data on Violation Rates at Intersection Approaches Using Regression Tree. In Proceeding of the 95 Transportation Research Board Meeting, (No. 16-1946).

Stoll, N., Glick, T., \& Figliozzi, M., (2016). Utilizing High Resolution Bus GPS Data to Visualize and Identify Congestion Hot-spots in Urban Arterials. Transportation Research Record: Journal of Transportation Research Board, (No. 16-6876).

Strathman, J., Kimpel, T., \& Callas, S. (2003). Headway Deviation Effects on Bus Passenger Loads: Analysis of Tri-Met's Archived AVL-APC Data, (No. TNW200301). Transportation Northwest, University of Washington.

Strathman, J., Dueker, K., Kimpel, T., Gerhart, R., Turner, K., Callas, S., \& Griffin, D. (2001). Bus Transit Operations Control: Review and an Experiment Involving TriMet's Automated Bus Dispatch System. Journal of Public Transportation, Vol. 41, No. 1: 1-26.

Tantiyanugulchai, S. (2004). “Arterial Performance Measurement using Transit Buses as Probe Vehicles." Portland State University, Master's Thesis.

TCRP Report 113: Using Archived AVL-APC Data to Improve Transit Performance and Management. (2006).

TCRP Report 88: A Guidebook for Developing a Transit Performance-Measurement System. (2003).

TCRP Report 165: Quality of Service. (2013). Transit Capacity and Quality of Service Manual. $3^{\text {rd }}$ Edition. 
TCRP Synthesis 73: AVL Systems for Bus Transit: Update. (2008).

Thornton, D., \& Coifman, B. (2015). Signal Progression Impacts on Transit Buses as Travel Time Probes. ASCE Journal of Transportation Engineering, 141(8).

TriMet. (2016). Service Annual Performance Report 2014-2016. Portland, OR: U.S.

Retrieved November 18, 2015, from: http://trimet.org/about/dashboard/index.htm TriMet Interactive Map. (2016). OpenStreetMap and Oregon Metro. Portland, OR: U.S. Retrieved October 21, 2015, from: http://ride.trimet.org/?tool=routes\&find=14\#/ 


\section{APPENDICES}

A: Abbreviations and Acronyms

AVL

5SR

APC

BDS

TriMet

GPS

DT

MPHPS

RMSE

SAS
Automatic Vehicle Location

5 Second Resolution

Automatic Passenger Count

Bus Dispatch System

Tri-County Metropolitan Organization in Portland, Oregon

Global Positioning System

Dwell Time

Miles per Hour per Second (Acceleration)

Root Mean Squared Error

Statistical Analysis Software 


\section{B: ArcGIS Map of BDS}

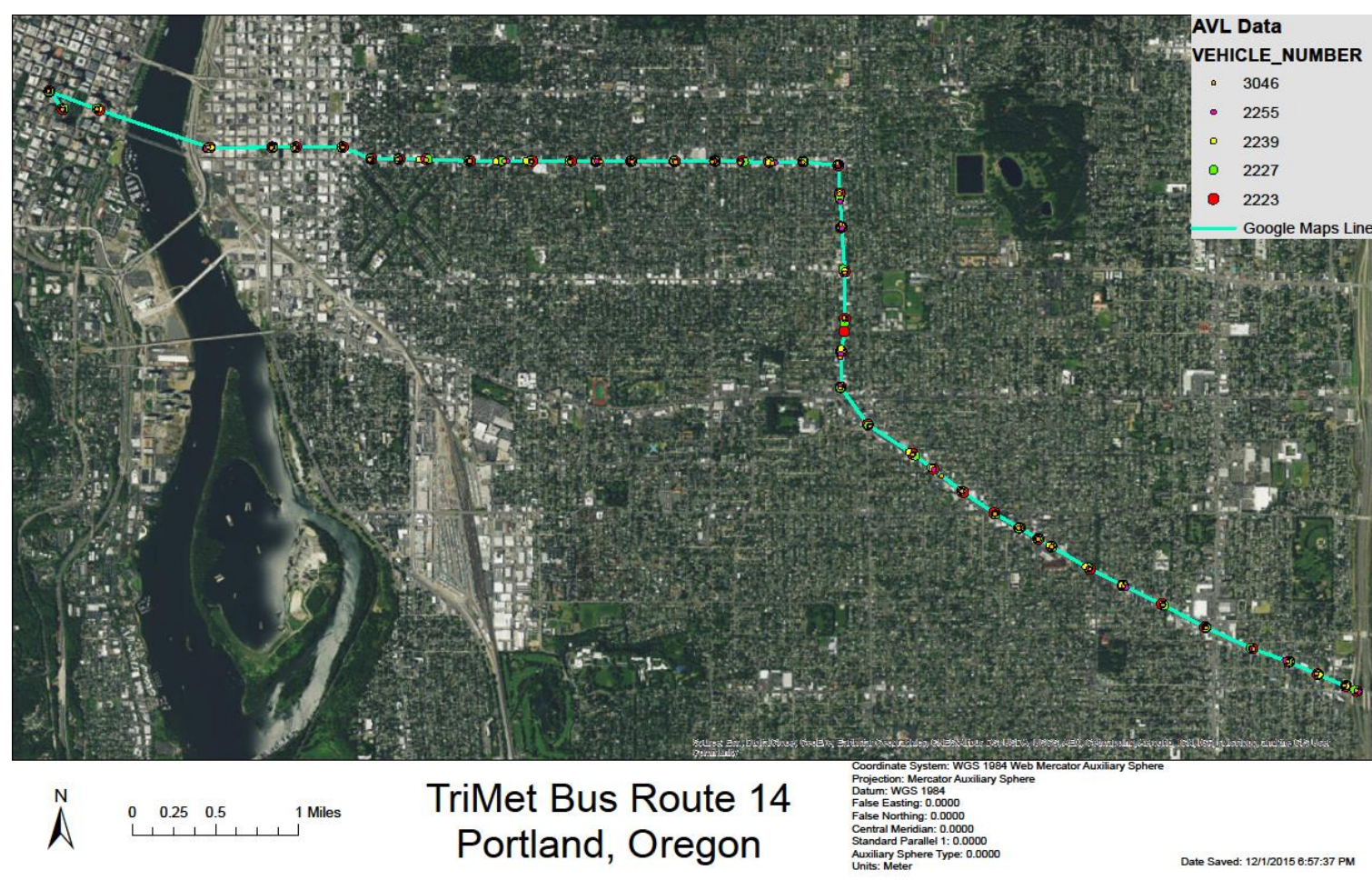


C: $\quad$ Regression Model Results

The SAS System

The GLM Procedure

Dependent Variable: Total_InterStop

\begin{tabular}{|c|c|c|c|c|c|}
\hline Source & DF & Sum of Squares & Mean Square & F Value & Pr $>$ F \\
\hline Model & 1 & 257746.4011 & 257746.4011 & 39.96 & $<.0001$ \\
\hline Error & 13 & 83856.9322 & 6450.5332 & & \\
\hline Corrected Total & 14 & 341603.3333 & & & \\
\hline
\end{tabular}

\begin{tabular}{|c|c|c|c|}
\hline R-Square & Coeff Var & Root MSE & Total_InterStop Mean \\
\hline 0.754520 & 4.816986 & 80.31521 & 1667.333 \\
\hline
\end{tabular}

\begin{tabular}{|l|c|c|c|c|c|}
\hline Source & DF & Type I SS & Mean Square & F Value & Pr $>$ F \\
\hline Signals & 1 & 257746.4011 & 257746.4011 & 39.96 & $<.0001$ \\
\hline
\end{tabular}

\begin{tabular}{|l|c|c|c|c|c|}
\hline Source & DF & Type III SS & Mean Square & F Value & Pr > F \\
\hline Signals & 1 & 257746.4011 & 257746.4011 & 39.96 & $<.0001$ \\
\hline
\end{tabular}

\begin{tabular}{|c|c|c|c|c|}
\hline Parameter & Estimate & $\begin{array}{c}\text { Standard } \\
\text { Error }\end{array}$ & $\mathbf{t}$ Value & $\operatorname{Pr}>|\mathbf{t}|$ \\
\hline Intercept & 977.3168498 & 111.1116592 & 8.80 & $<.0001$ \\
\hline Signals & 84.1483516 & 13.3121167 & 6.32 & $<.0001$ \\
\hline
\end{tabular}

The SAS System

The GLM Procedure

\begin{tabular}{|l|l|}
\hline Number of Observations Read & 18 \\
\hline Number of Observations Used & 18 \\
\hline
\end{tabular}

The SAS System 
The GLM Procedure

Dependent Variable: Total_InterStop

\begin{tabular}{|c|c|c|c|c|c|}
\hline Source & DF & Sum of Squares & Mean Square & F Value & Pr $>$ F \\
\hline Model & 1 & 190850.2092 & 190850.2092 & 65.91 & $<.0001$ \\
\hline Error & 16 & 46331.5686 & 2895.7230 & & \\
\hline Corrected Total & 17 & 237181.7778 & & & \\
\hline
\end{tabular}

\begin{tabular}{|c|c|c|c|}
\hline R-Square & Coeff Var & Root MSE & Total_InterStop Mean \\
\hline 0.804658 & 3.433829 & 53.81192 & 1567.111 \\
\hline
\end{tabular}

\begin{tabular}{|l|c|c|c|c|c|}
\hline Source & DF & Type I SS & Mean Square & F Value & $\operatorname{Pr}>$ F \\
\hline Signals & 1 & 190850.2092 & 190850.2092 & 65.91 & $<.0001$ \\
\hline
\end{tabular}

\begin{tabular}{|l|c|c|c|c|c|}
\hline Source & DF & Type III SS & Mean Square & F Value & $\operatorname{Pr}>$ F \\
\hline Signals & 1 & 190850.2092 & 190850.2092 & 65.91 & $<.0001$ \\
\hline
\end{tabular}

\begin{tabular}{|c|c|c|c|c|}
\hline Parameter & Estimate & $\begin{array}{c}\text { Standard } \\
\text { Error }\end{array}$ & $\mathbf{t}$ Value & $\operatorname{Pr}>|\mathbf{t}|$ \\
\hline Intercept & 1242.450980 & 41.95408653 & 29.61 & $<.0001$ \\
\hline Signals & 49.947712 & 6.15244575 & 8.12 & $<.0001$ \\
\hline
\end{tabular}

The SAS System

The GLM Procedure

\begin{tabular}{|l|l|}
\hline Number of Observations Read & 12 \\
\hline Number of Observations Used & 12 \\
\hline
\end{tabular}

The SAS System

The GLM Procedure

Dependent Variable: Total_InterStop 


\begin{tabular}{|c|c|c|c|c|c|}
\hline Source & DF & Sum of Squares & Mean Square & F Value & Pr $>$ F \\
\hline Model & 1 & 72613.13995 & 72613.13995 & 31.25 & 0.0002 \\
\hline Error & 10 & 23234.52672 & 2323.45267 & & \\
\hline Corrected Total & 11 & 95847.66667 & & & \\
\hline
\end{tabular}

\begin{tabular}{|c|c|c|c|}
\hline R-Square & Coeff Var & Root MSE & Total_InterStop Mean \\
\hline 0.757589 & 2.914575 & 48.20221 & 1653.833 \\
\hline
\end{tabular}

\begin{tabular}{|c|c|c|c|c|c|}
\hline Source & DF & Type I SS & Mean Square & F Value & Pr $>$ F \\
\hline Signals & 1 & 72613.13995 & 72613.13995 & 31.25 & 0.0002 \\
\hline
\end{tabular}

\begin{tabular}{|l|c|c|c|c|c|}
\hline Source & DF & Type III SS & Mean Square & F Value & Pr $>$ F \\
\hline Signals & 1 & 72613.13995 & 72613.13995 & 31.25 & 0.0002 \\
\hline
\end{tabular}

\begin{tabular}{|c|c|c|c|c|}
\hline Parameter & Estimate & $\begin{array}{c}\text { Standard } \\
\text { Error }\end{array}$ & t Value & $\operatorname{Pr}>|\mathbf{t}|$ \\
\hline Intercept & 1334.400763 & 58.80964706 & 22.69 & $<.0001$ \\
\hline Signals & 40.778626 & 7.29443896 & 5.59 & 0.0002 \\
\hline
\end{tabular}

The SAS System

The GLM Procedure

\begin{tabular}{|l|l|}
\hline Number of Observations Read & 7 \\
\hline Number of Observations Used & 7 \\
\hline
\end{tabular}

The SAS System

The GLM Procedure

Dependent Variable: Total_InterStop

\begin{tabular}{|c|c|c|c|c|c|}
\hline Source & DF & Sum of Squares & Mean Square & F Value & Pr $>$ F \\
\hline Model & 1 & 23147.52381 & 23147.52381 & 9.00 & 0.0301 \\
\hline
\end{tabular}




\begin{tabular}{|c|c|c|c|c|c|}
\hline Error & 5 & 12855.33333 & 2571.06667 & & \\
\hline Corrected Total & 6 & 36002.85714 & & & \\
\hline
\end{tabular}

\begin{tabular}{|c|c|c|c|}
\hline R-Square & Coeff Var & Root MSE & Total_InterStop Mean \\
\hline 0.642936 & 3.449702 & 50.70569 & 1469.857 \\
\hline
\end{tabular}

\begin{tabular}{|c|c|c|c|c|c|}
\hline Source & DF & Type I SS & Mean Square & F Value & Pr $>$ F \\
\hline Signals & 1 & 23147.52381 & 23147.52381 & 9.00 & 0.0301 \\
\hline
\end{tabular}

\begin{tabular}{|c|c|c|c|c|c|}
\hline Source & DF & Type III SS & Mean Square & F Value & $\operatorname{Pr}>$ F \\
\hline Signals & 1 & 23147.52381 & 23147.52381 & 9.00 & 0.0301 \\
\hline
\end{tabular}

\begin{tabular}{|c|c|c|c|c|}
\hline Parameter & Estimate & $\begin{array}{c}\text { Standard } \\
\text { Error }\end{array}$ & $\begin{array}{c}\mathbf{t} \\
\text { Value }\end{array}$ & $\begin{array}{c}\text { Pr }> \\
|\mathbf{t}|\end{array}$ \\
\hline Intercept & 1287.916667 & 63.59303115 & 20.25 & $<.0001$ \\
\hline Signals & 41.083333 & 13.69210032 & 3.00 & 0.0301 \\
\hline
\end{tabular}


D: Decision Tree Output

RUN 1:

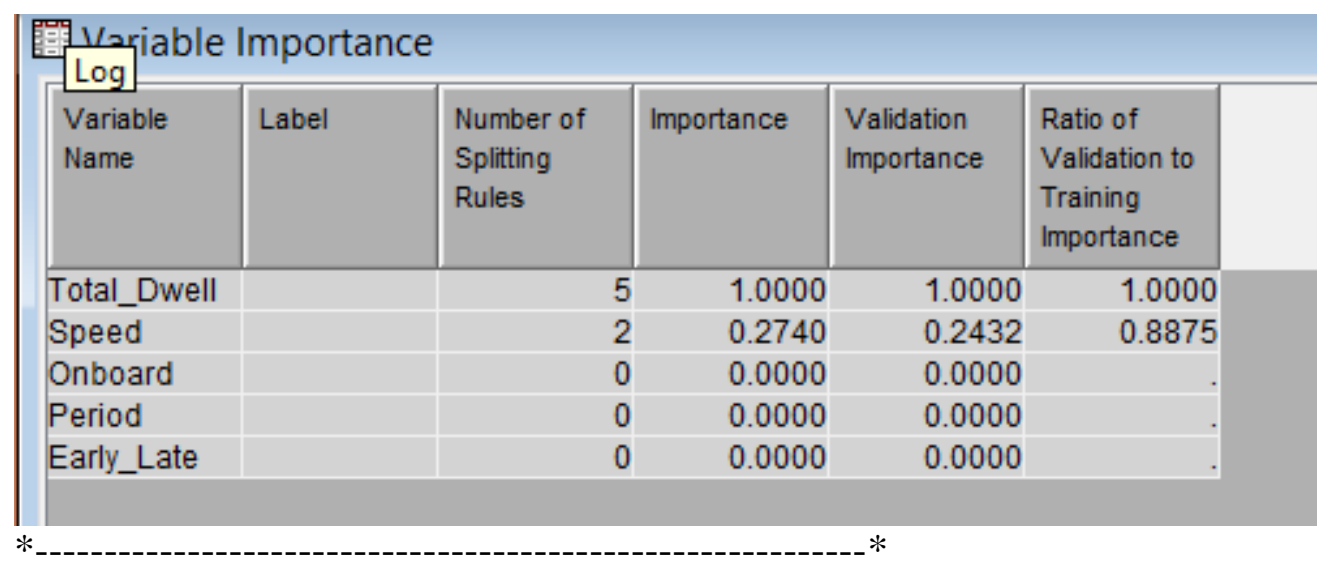

Node $=4$

$*$

if Total_Dwell $<85.5$

then

Tree Node Identifier $=4$

Number of Observations $=45$

Predicted: Seconds $=315.37777778$

*

Node $=7$

$*$

if Total_Dwell $>=225.5$

then

Tree Node Identifier $=7$

Number of Observations $=37$

Predicted: Seconds $=489.51351351$

*

if Total_Dwell $<150.5$ AND Total_Dwell $>=85.5$ or MISSING

AND Speed $>=24.5$

then

Tree Node Identifier $=9$

Number of Observations $=28$

Predicted: Seconds $=341.32142857$

* ${ }^{*}$ Node $=10$

if Total_Dwell $<225.5$ AND Total_Dwell $>=150.5$ or MISSING 
AND Speed $<22.5$

then

Tree Node Identifier $=10$

Number of Observations $=29$

Predicted: Seconds $=457.93103448$

Node $=12$
*-of 12
if Total_Dwell < 123.5 AND Total_Dwell >= 85.5
AND Speed $<24.5$ or MISSING
then
Tree Node Identifier $=12$
Number of Observations $=42$
Predicted: Seconds $=371.45238095$
*
Node $=13$

if Total_Dwell $<150.5$ AND Total_Dwell $>=123.5$ or MISSING

AND Speed $<24.5$ or MISSING

then

Tree Node Identifier $=13$

Number of Observations $=43$

Predicted: Seconds $=391.39534884$

*ode $=14$

if Total_Dwell $<192.5$ AND Total_Dwell $>=150.5$ or MISSING

AND Speed $>=22.5$ or MISSING

then

Tree Node Identifier $=14$

Number of Observations $=71$

Predicted: Seconds $=411.30985915$

*

Node $=15$

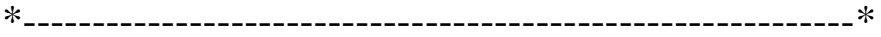

if Total_Dwell $<225.5$ AND Total_Dwell $>=192.5$

AND Speed $>=22.5$ or MISSING

then

Tree Node Identifier $=15$

Number of Observations $=44$

Predicted: Seconds $=438.43181818$ 


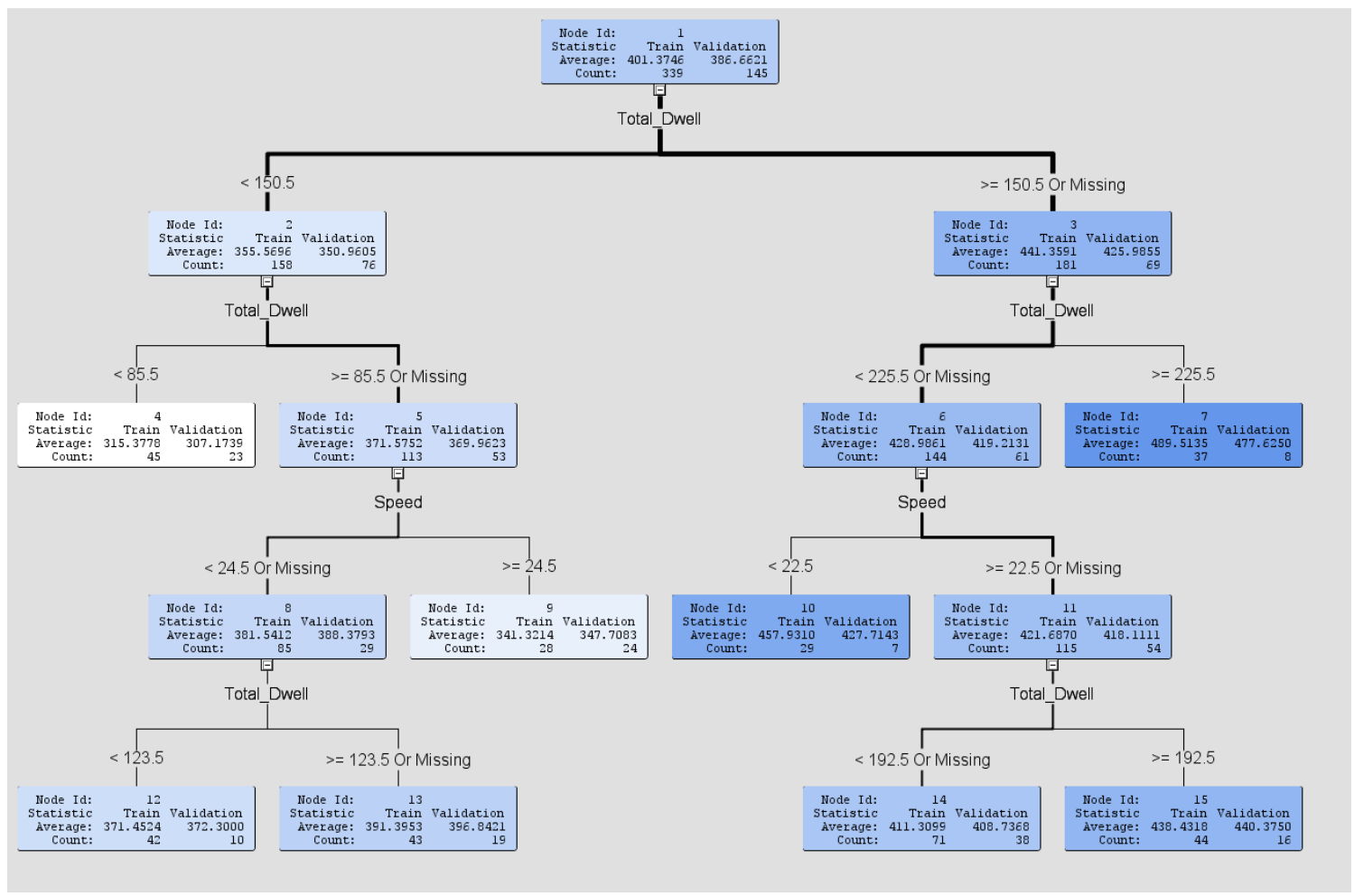


RUN 2:

\begin{tabular}{|c|c|c|c|c|c|}
\hline $\begin{array}{l}\text { Variable } \\
\text { Name }\end{array}$ & Label & $\begin{array}{l}\text { Number of } \\
\text { Splitting } \\
\text { Rules }\end{array}$ & Importance & $\begin{array}{l}\text { Validation } \\
\text { Importance }\end{array}$ & $\begin{array}{l}\text { Ratio of } \\
\text { Validation to } \\
\text { Training } \\
\text { Importance }\end{array}$ \\
\hline Period & & & 1.0000 & 1.0000 & 1.0000 \\
\hline Onboard & & & 0.6258 & 0.7831 & 1.2513 \\
\hline Speed & & & 0.4621 & 0.0000 & 0.0000 \\
\hline Early_Late & & & 0.0000 & 0.0000 & \\
\hline
\end{tabular}

Node $=2$

if Period IS ONE OF: 1,5

then

Tree Node Identifier $=2$

Number of Observations $=46$

Predicted: Seconds $=331.17391304$

*

Node $=4$

*--

if Period IS ONE OF: 2, 3, 4 or MISSING

AND Onboard $<14.5$

then

Tree Node Identifier $=4$

Number of Observations $=77$

Predicted: Seconds $=385.44155844$

$*$

Node $=8$

if Speed $<22.5$

AND Period IS ONE OF: 2, 3, 4 or MISSING

AND Onboard $>=14.5$ or MISSING

then

Tree Node Identifier $=8$

Number of Observations $=45$

Predicted: Seconds $=454.06666667$

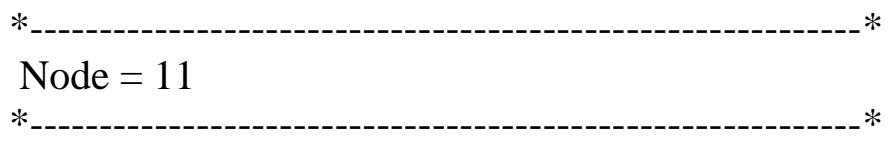

if Speed $>=22.5$ or MISSING

AND Period IS ONE OF: 2, 3, 4 or MISSING

AND Onboard $>=38.5$ 


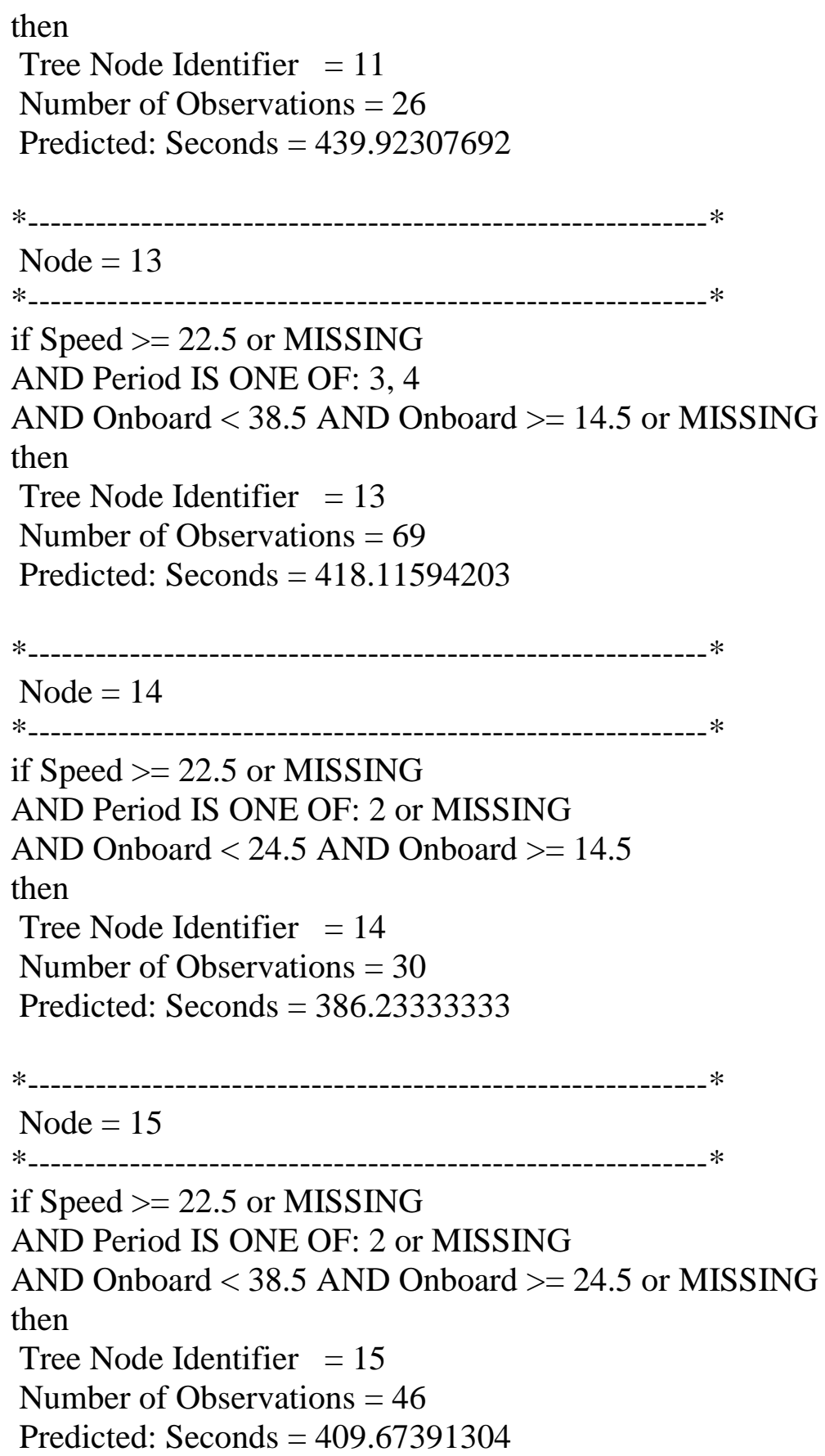




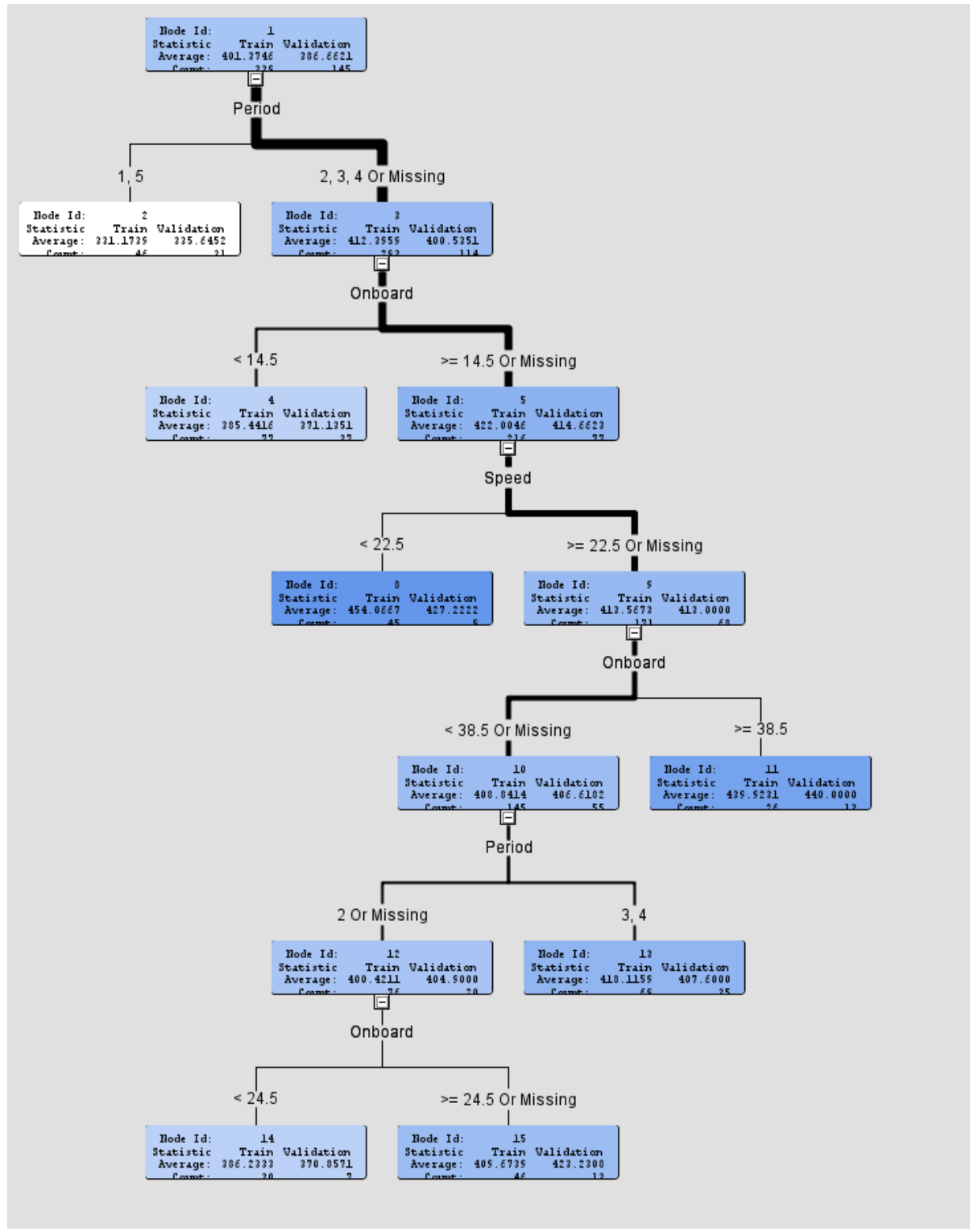




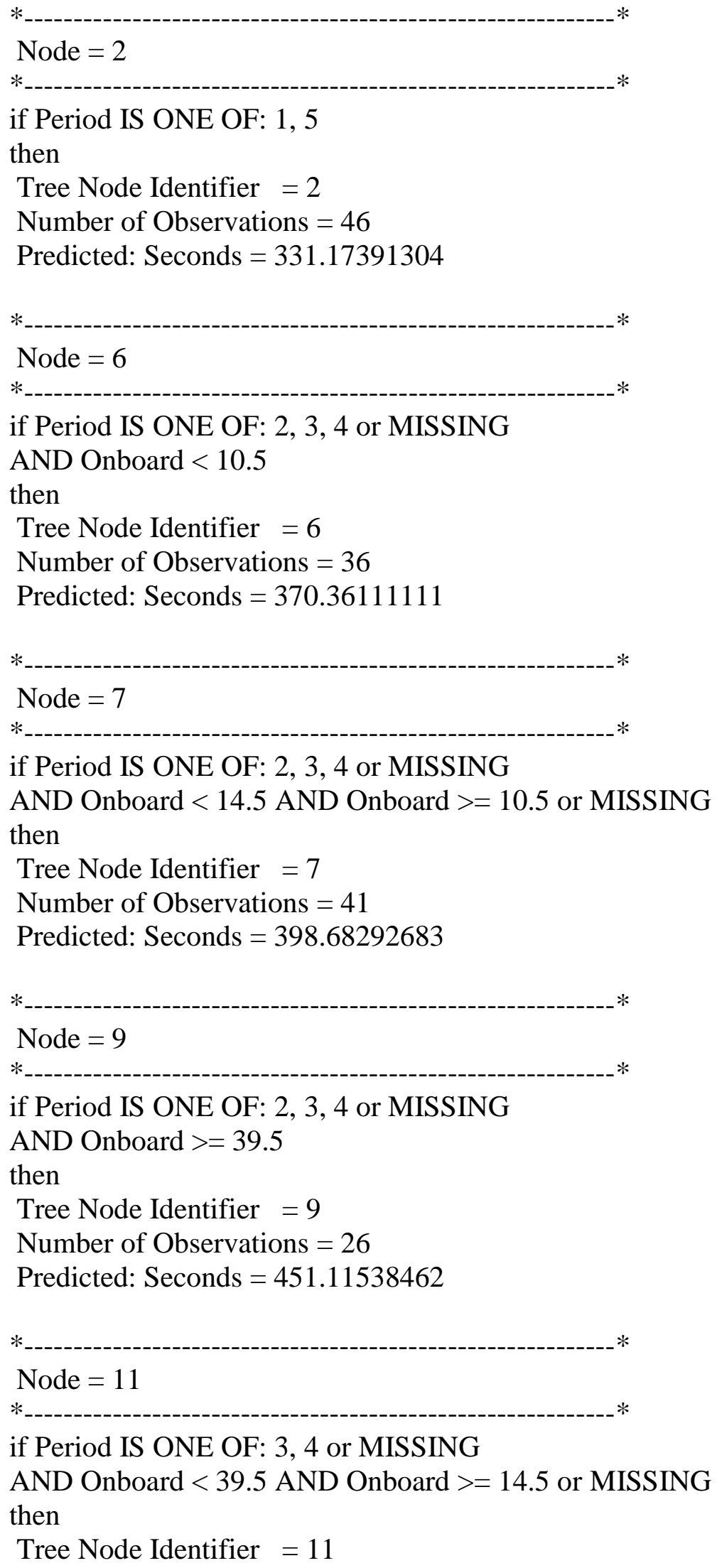


Number of Observations $=98$

Predicted: Seconds $=430.47959184$

$*$

Node $=12$

*

if Period IS ONE OF: 2

AND Onboard $<24.5$ AND Onboard $>=14.5$

then

Tree Node Identifier $=12$

Number of Observations $=33$

Predicted: Seconds $=389.54545455$

*

Node $=16$

if Period IS ONE OF: 2

AND Onboard $<31.5$ AND Onboard $>=24.5$

then

Tree Node Identifier $=16$

Number of Observations $=29$

Predicted: Seconds $=419.62068966$

$*$

Node $=17$

*.

if Period IS ONE OF: 2

AND Onboard $<39.5$ AND Onboard $>=31.5$ or MISSING

then

Tree Node Identifier $=17$

Number of Observations $=30$

Predicted: Seconds $=407.1$

RUN 3:

\begin{tabular}{|l|l|l|l|l|l|}
\hline $\begin{array}{l}\text { Variable } \\
\text { Name }\end{array}$ & Label & $\begin{array}{l}\text { Number of } \\
\text { Splitting } \\
\text { Rules }\end{array}$ & Importance & $\begin{array}{l}\text { Validation } \\
\text { Importance }\end{array}$ & $\begin{array}{l}\text { Ratio of } \\
\text { Validation to } \\
\text { Training } \\
\text { Importance }\end{array}$ \\
\hline Period & & 2 & 1.0000 & 1.0000 & 1.0000 \\
\hline Onboard & & 5 & 0.6666 & 0.9140 & 1.3710 \\
\hline Early_Late & & 0 & 0.0000 & 0.0000 & \\
\hline
\end{tabular}




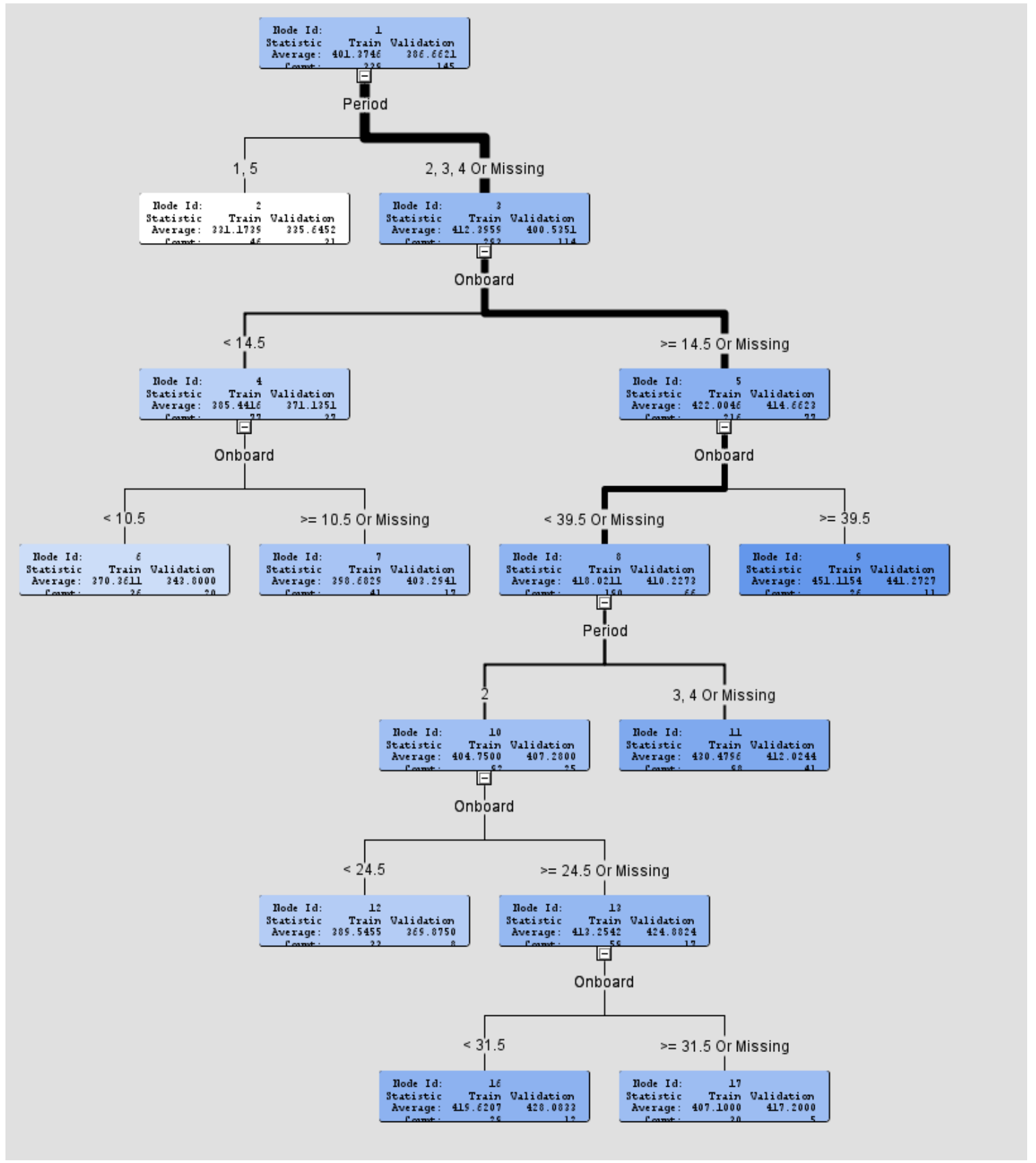




\section{E: Newell Dwell Time Model Output}

\section{SUMMARY OUTPUT}

\begin{tabular}{lr}
\hline \multicolumn{2}{c}{ Regression Statistics } \\
\hline Multiple R & 0.636 \\
R Square & 0.404 \\
Adjusted R Square & 0.404 \\
Standard Error & 10.044 \\
Observations & 2511 \\
\hline
\end{tabular}

ANOVA

\begin{tabular}{lrrrrr}
\hline & $d f$ & $S S$ & \multicolumn{1}{c}{$M S$} & \multicolumn{1}{c}{ F } & Significance $F$ \\
\hline Regression & 2 & 171494.1 & 85747.1 & 850.0 & 0.0 \\
Residual & 2508 & 252991.3 & 100.9 & & \\
Total & 2510 & 424485.4 & & & \\
\hline
\end{tabular}

\begin{tabular}{|c|c|c|c|c|c|c|c|c|}
\hline & Coefficients & $\begin{array}{l}\text { Standard } \\
\text { Error }\end{array}$ & $t$ Stat & P-value & $\begin{array}{l}\text { Lower } \\
95 \%\end{array}$ & $\begin{array}{l}\text { Upper } \\
95 \%\end{array}$ & $\begin{array}{l}\text { Lower } \\
95.0 \%\end{array}$ & $\begin{array}{l}\text { Upper } \\
95.0 \%\end{array}$ \\
\hline $\begin{array}{l}\text { Intercept } \\
\text { X Variable }\end{array}$ & 19.33 & 0.33 & 42.38 & $\begin{array}{l}1.7 \mathrm{E}- \\
296 \\
1.2 \mathrm{E}-\end{array}$ & 19.14 & 19.41 & 19.14 & 19.41 \\
\hline $\begin{array}{l}1 \\
\text { X Variable }\end{array}$ & 3.61 & 0.13 & 27.38 & $\begin{array}{l}144 \\
2.4 \mathrm{E}-\end{array}$ & 3.35 & 3.87 & 3.35 & 3.87 \\
\hline 2 & 1.38 & 0.04 & 34.75 & 216 & 1.31 & 1.46 & 1.31 & 1.46 \\
\hline
\end{tabular}


F: $\quad$ Sample of 1 Second Intervals

\begin{tabular}{|c|c|c|c|c|c|c|c|}
\hline ACT_TIME & Actual Time & Distance & Speed & Gap Time & 1 Second & Increments & Trip Distance \\
\hline 29882 & 8:18:02 & 0 & 0 & 0 & 29882 & 0.0000 & 0.0000 \\
\hline 29887 & $8: 18: 07$ & 0.01849 & 13.3117 & 5 & 29883 & 0.0037 & 0.0037 \\
\hline 29892 & $8: 18: 12$ & 0.02656 & 19.1213 & 5 & 29884 & 0.0037 & 0.0074 \\
\hline 29897 & $8: 18: 17$ & 0.01259 & 9.06351 & 5 & 29885 & 0.0037 & 0.0111 \\
\hline 29912 & $8: 18: 32$ & 0.01639 & 3.93326 & 15 & 29886 & 0.0037 & 0.0148 \\
\hline 29917 & $8: 18: 37$ & 0.0191 & 13.752 & 5 & 29887 & 0.0037 & 0.0185 \\
\hline 29922 & $8: 18: 42$ & 0.00924 & 6.65606 & 5 & 29888 & 0.0053 & 0.0238 \\
\hline 29947 & $8: 19: 07$ & 0.01317 & 1.89629 & 25 & 29889 & 0.0053 & 0.0291 \\
\hline 29952 & $8: 19: 12$ & 0.03032 & 21.827 & 5 & 29890 & 0.0053 & 0.0344 \\
\hline 29957 & $8: 19: 17$ & 0.03888 & 27.994 & 5 & 29891 & 0.0053 & 0.0397 \\
\hline 29962 & $8: 19: 22$ & 0.04103 & 29.5421 & 5 & 29892 & 0.0053 & 0.0450 \\
\hline 29967 & $8: 19: 27$ & 0.04119 & 29.6586 & 5 & 29893 & 0.0025 & 0.0476 \\
\hline 29972 & $8: 19: 32$ & 0.03938 & 28.3501 & 5 & 29894 & 0.0025 & 0.0501 \\
\hline 29977 & $8: 19: 37$ & 0.03601 & 25.9265 & 5 & 29895 & 0.0025 & 0.0526 \\
\hline 29982 & $8: 19: 42$ & 0.03457 & 24.8908 & 5 & 29896 & 0.0025 & 0.0551 \\
\hline 29987 & $8: 19: 47$ & 0.03536 & 25.4594 & 5 & 29897 & 0.0025 & 0.0576 \\
\hline 29992 & $8: 19: 52$ & 0.0357 & 25.704 & 5 & 29898 & 0.0011 & 0.0587 \\
\hline 29997 & $8: 19: 57$ & 0.02896 & 20.8481 & 5 & 29899 & 0.0011 & 0.0598 \\
\hline 30017 & $8: 20: 17$ & 0.03307 & 5.95322 & 20 & 29900 & 0.0011 & 0.0609 \\
\hline 30022 & $8: 20: 22$ & 0.02903 & 20.9007 & 5 & 29901 & 0.0011 & 0.0620 \\
\hline 30027 & $8: 20: 27$ & 0.02885 & 20.7728 & 5 & 29902 & 0.0011 & 0.0631 \\
\hline 30032 & $8: 20: 32$ & 0.02643 & 19.0322 & 5 & 29903 & 0.0011 & 0.0642 \\
\hline 30037 & $8: 20: 37$ & 0.02067 & 14.8859 & 5 & 29904 & 0.0011 & 0.0653 \\
\hline 30042 & $8: 20: 42$ & 0.01103 & 7.93916 & 5 & 29905 & 0.0011 & 0.0664 \\
\hline 30047 & $8: 20: 47$ & 0.00956 & 6.88679 & 5 & 29906 & 0.0011 & 0.0675 \\
\hline 30052 & $8: 20: 52$ & 0.01914 & 13.7813 & 5 & 29907 & 0.0011 & 0.0686 \\
\hline 30057 & $8: 20: 57$ & 0.02407 & 17.3275 & 5 & 29908 & 0.0011 & 0.0697 \\
\hline 30062 & $8: 21: 02$ & 0.01955 & 14.0784 & 5 & 29909 & 0.0011 & 0.0707 \\
\hline 30117 & $8: 21: 57$ & 0.01774 & 1.16085 & 55 & 29910 & 0.0011 & 0.0718 \\
\hline 30122 & $8: 22: 02$ & 0.03252 & 23.4147 & 5 & 29911 & 0.0011 & 0.0729 \\
\hline 30127 & $8: 22: 07$ & 0.03881 & 27.9398 & 5 & 29912 & 0.0011 & 0.0740 \\
\hline 30132 & $8: 22: 12$ & 0.04103 & 29.5414 & 5 & 29913 & 0.0038 & 0.0778 \\
\hline 30137 & $8: 22: 17$ & 0.03827 & 27.5553 & 5 & 29914 & 0.0038 & 0.0817 \\
\hline 30142 & $8: 22: 22$ & 0.03032 & 21.8322 & 5 & 29915 & 0.0038 & 0.0855 \\
\hline 30147 & $8: 22: 27$ & 0.01003 & 7.2202 & 5 & 29916 & 0.0038 & 0.0893 \\
\hline 30162 & $8: 22: 42$ & 0.01479 & 3.55031 & 15 & 29917 & 0.0038 & 0.0931 \\
\hline
\end{tabular}




\begin{tabular}{|c|c|c|c|c|c|c|}
\hline 1 Second & 5 SR Increment & 5SR Cum. Dist. & Stop Level Data & Interpolation & Stop Cum. Dist. & Difference \\
\hline 29370 & 0 & 0 & 0 & 0 & 0.000 & 0 \\
\hline 29371 & 0.00315 & 0.00315 & 0.002 & 0.002 & 0.002 & -0.001 \\
\hline 29372 & 0.00315 & 0.00629 & & 0 & 0.003 & -0.003 \\
\hline 29373 & 0.00315 & 0.00944 & & 0 & 0.005 & -0.004 \\
\hline 29374 & 0.00315 & 0.01258 & & 0 & 0.007 & -0.006 \\
\hline 29375 & 0.00315 & 0.01573 & & 0 & 0.009 & -0.007 \\
\hline 29376 & 0.00122 & 0.01695 & & 0 & 0.010 & -0.007 \\
\hline 29377 & 0.00122 & 0.01817 & & 0 & 0.012 & -0.006 \\
\hline 29378 & 0.00122 & 0.01938 & & 0 & 0.014 & -0.006 \\
\hline 29379 & 0.00122 & 0.02060 & & 0 & 0.016 & -0.005 \\
\hline 29380 & 0.00122 & 0.02182 & & 0 & 0.017 & -0.005 \\
\hline 29381 & 0.00000 & 0.02182 & & 0 & 0.019 & -0.003 \\
\hline 29382 & 0.00000 & 0.02182 & & 0 & 0.021 & -0.001 \\
\hline 29383 & 0.00000 & 0.02182 & & 0 & 0.022 & 0.001 \\
\hline 29384 & 0.00000 & 0.02182 & & 0 & 0.024 & 0.002 \\
\hline 29385 & 0.00000 & 0.02182 & & 0 & 0.026 & 0.004 \\
\hline 29386 & 0.00000 & 0.02182 & & 0 & 0.028 & 0.006 \\
\hline 29387 & 0.00000 & 0.02182 & & 0 & 0.029 & 0.008 \\
\hline 29388 & 0.00000 & 0.02182 & & 0 & 0.031 & 0.009 \\
\hline 29389 & 0.00000 & 0.02182 & & 0 & 0.033 & 0.011 \\
\hline 29390 & 0.00000 & 0.02182 & & 0 & 0.035 & 0.013 \\
\hline 29391 & 0.00159 & 0.02341 & & 0 & 0.036 & 0.013 \\
\hline 29392 & 0.00159 & 0.02499 & & 0 & 0.038 & 0.013 \\
\hline 29393 & 0.00159 & 0.02658 & & 0 & 0.040 & 0.013 \\
\hline 29394 & 0.00159 & 0.02816 & & 0 & 0.042 & 0.013 \\
\hline 29395 & 0.00159 & 0.02975 & & 0 & 0.043 & 0.013 \\
\hline 29396 & 0.00450 & 0.03425 & & 0 & 0.045 & 0.011 \\
\hline 29397 & 0.00450 & 0.03876 & & 0 & 0.047 & 0.008 \\
\hline 29398 & 0.00450 & 0.04326 & & 0 & 0.048 & 0.005 \\
\hline 29399 & 0.00450 & 0.04777 & & 0 & 0.050 & 0.002 \\
\hline 29400 & 0.00450 & 0.05227 & & 0 & 0.052 & 0.000 \\
\hline 29401 & 0.00351 & 0.05578 & & 0 & 0.054 & -0.002 \\
\hline 29402 & 0.00351 & 0.05928 & & 0 & 0.055 & -0.004 \\
\hline 29403 & 0.00200 & 0.06128 & & 0 & 0.057 & -0.004 \\
\hline 29404 & 0.00200 & 0.06328 & & 0 & 0.059 & -0.004 \\
\hline 29405 & 0.00000 & 0.06400 & & 0 & 0.061 & -0.003 \\
\hline 29406 & 0.00000 & 0.06400 & & 0 & 0.062 & -0.002 \\
\hline 29407 & 0.00000 & 0.06400 & 0.064 & 0.064 & 0.064 & 0 \\
\hline 29408 & 0.00000 & 0.06400 & & 0 & 0.064 & 0 \\
\hline 29409 & 0.00000 & 0.06400 & & 0 & 0.064 & 0 \\
\hline
\end{tabular}




\section{G: $\quad$ Sample of 5SR Signals Encountered}

\begin{tabular}{|c|c|c|c|c|c|c|c|c|c|c|c|c|}
\hline VEHICLE_ID & OPD_DATE & CT_TIME & GPS_LOI & NGPS_LAT & TActual Time & Distance & Trip Distance & Speed & Gap Time & Stop Distance & Dwell & Signal \\
\hline 2001 & $130 \mathrm{CT} 2014$ & 65816 & -122.57 & 45.479 & 18:16:56 & 0.000 & 0.000 & 0.0 & 0 & 0.000 & & \\
\hline 2001 & $130 \mathrm{CT} 2014$ & 65821 & -122.57 & 45.4791 & 18:17:01 & 0.017 & 0.017 & 12.6 & 5 & 0.000 & & \\
\hline 2001 & $130 С T 2014$ & 65826 & -122.57 & 45.4792 & 18:17:06 & 0.032 & 0.050 & 23.3 & 5 & 0.000 & & \\
\hline 2001 & $130 \mathrm{CT} 2014$ & 65831 & -122.57 & 45.4794 & 18:17:11 & 0.030 & 0.079 & 21.4 & 5 & 0.000 & & \\
\hline 2001 & 13ОСТ2014 & 65866 & -122.57 & 45.4795 & 18:17:46 & 0.019 & 0.099 & 2.0 & 35 & 0.064 & 30 & \\
\hline 2001 & $130 \mathrm{CT} 2014$ & 65871 & -122.57 & 45.4797 & 18:17:51 & 0.028 & 0.127 & 20.5 & 5 & 0.064 & & \\
\hline 2001 & 130 СТ2014 & 65876 & -122.57 & 45.4799 & $18: 17: 56$ & 0.035 & 0.163 & 25.5 & 5 & 0.064 & & \\
\hline 2001 & $130 \mathrm{CT} 2014$ & 65881 & -122.57 & 45.4801 & 18:18:01 & 0.037 & 0.199 & 26.4 & 5 & 0.064 & & \\
\hline 2001 & $130 \mathrm{CT} 2014$ & 65886 & -122.57 & 45.4803 & 18:18:06 & 0.036 & 0.236 & 26.2 & 5 & 0.202 & 0 & \\
\hline 2001 & $130 \mathrm{CT} 2014$ & 65891 & -122.57 & 45.4806 & $18: 18: 11$ & 0.038 & 0.274 & 27.1 & 5 & 0.202 & & \\
\hline 2001 & $130 С T 2014$ & 65896 & -122.57 & 45.4808 & $18: 18: 16$ & 0.038 & 0.312 & 27.7 & 5 & 0.202 & & \\
\hline 2001 & $130 С T 2014$ & 65901 & -122.57 & 45.481 & $18: 18: 21$ & 0.037 & 0.349 & 26.7 & 5 & 0.202 & & \\
\hline 2001 & 130 СТ2014 & 65906 & -122.57 & 45.4812 & $18: 18: 26$ & 0.037 & 0.387 & 27.0 & 5 & 0.316 & 0 & \\
\hline 2001 & 130 СТ2014 & 65911 & -122.57 & 45.4814 & 18:18:31 & 0.039 & 0.425 & 27.9 & 5 & 0.316 & & \\
\hline 2001 & 130 СТ 2014 & 65916 & -122.57 & 45.4816 & $18: 18: 36$ & 0.039 & 0.464 & 28.1 & 5 & 0.316 & & \\
\hline 2001 & $130 \mathrm{CT} 2014$ & 65921 & -122.58 & 45.4818 & 18:18:41 & 0.039 & 0.503 & 28.1 & 5 & 0.316 & & \\
\hline 2001 & 130 СТ2014 & 65926 & -122.58 & 45.482 & $18: 18: 46$ & 0.038 & 0.542 & 27.5 & 5 & 0.482 & 0 & \\
\hline 2001 & 130 СТ2014 & 65931 & -122.58 & 45.4821 & $18: 18: 51$ & 0.038 & 0.579 & 27.3 & 5 & 0.482 & & \\
\hline 2001 & 130 СТ 2014 & 65936 & -122.58 & 45.4823 & $18: 18: 56$ & 0.033 & 0.613 & 24.0 & 5 & 0.482 & & \\
\hline 2001 & 130 СТ2014 & 65941 & -122.58 & 45.4825 & 18:19:01 & 0.029 & 0.642 & 20.9 & 5 & 0.482 & & \\
\hline 2001 & $130 С T 2014$ & 65946 & -122.58 & 45.4826 & 18:19:06 & 0.019 & 0.660 & 13.4 & 5 & 0.482 & & \\
\hline 2001 & $130 \mathrm{CT} 2014$ & 65996 & -122.58 & 45.4827 & 18:19:56 & 0.002 & 0.662 & 0.1 & 50 & 0.700 & & 1 \\
\hline 2001 & 130 СТ2014 & 66001 & -122.58 & 45.4827 & $18: 20: 01$ & 0.010 & 0.672 & 7.3 & 5 & 0.700 & & \\
\hline 2001 & 130 СТ 2014 & 66006 & -122.58 & 45.4828 & $18: 20: 06$ & 0.006 & 0.679 & 4.5 & 5 & 0.700 & & \\
\hline 2001 & 130 СТ2014 & 66011 & -122.58 & 45.4829 & $18: 20: 11$ & 0.017 & 0.696 & 12.5 & 5 & 0.700 & & \\
\hline 2001 & 130 СТ2014 & 66015 & -122.58 & 45.4831 & $18: 20: 15$ & 0.027 & 0.723 & 24.3 & 4 & 0.700 & & \\
\hline 2001 & 130 CT2014 & 66045 & -122.58 & 45.4834 & $18: 20: 45$ & 0.040 & 0.763 & 4.8 & 30 & 0.700 & & 1 \\
\hline 2001 & 130 СТ2014 & 66050 & -122.58 & 45.4836 & $18: 20: 50$ & 0.036 & 0.799 & 26.1 & 5 & 0.700 & & \\
\hline 2001 & 130 СТ2014 & 66055 & -122.58 & 45.4839 & $18: 20: 55$ & 0.037 & 0.837 & 26.8 & 5 & 0.700 & & \\
\hline 2001 & 130 СТ2014 & 66060 & -122.58 & 45.4841 & $18: 21: 00$ & 0.039 & 0.875 & 27.9 & 5 & 0.700 & & \\
\hline 2001 & 130 СТ2014 & 66065 & -122.58 & 45.4844 & 18:21:05 & 0.038 & 0.914 & 27.6 & 5 & 0.700 & & \\
\hline 2001 & 130 СТ2014 & 66070 & -122.58 & 45.4847 & $18: 21: 10$ & 0.037 & 0.951 & 26.9 & 5 & 0.700 & & \\
\hline 2001 & $130 \mathrm{CT} 2014$ & 66075 & -122.58 & 45.4849 & 18:21:15 & 0.038 & 0.989 & 27.5 & 5 & 0.902 & 0 & \\
\hline 2001 & $130 \mathrm{CT} 2014$ & 66080 & -122.59 & 45.4852 & $18: 21: 20$ & 0.039 & 1.029 & 28.3 & 5 & 0.902 & & \\
\hline
\end{tabular}




\section{H: New Inter-Stop Models}

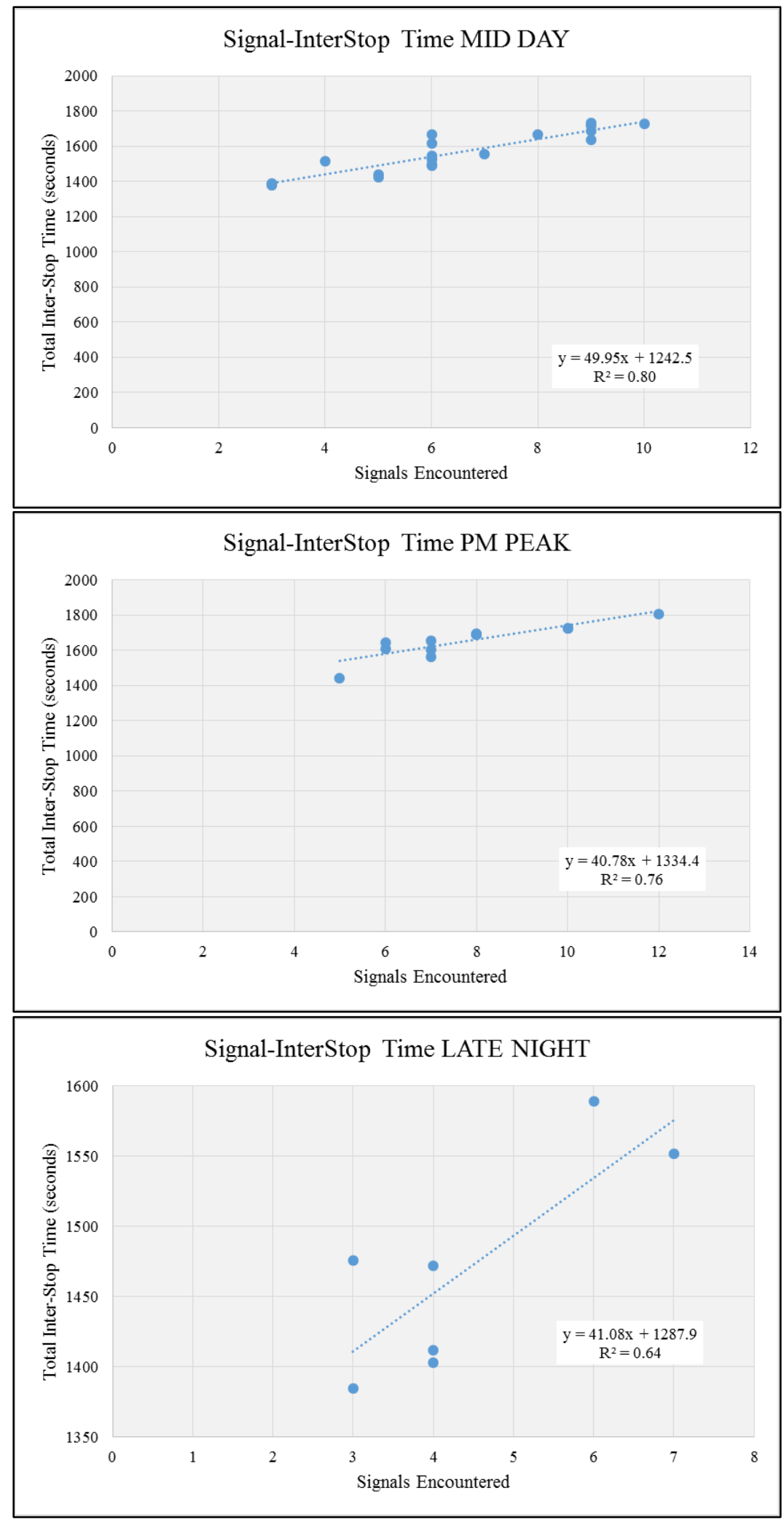

